\title{
Observation and integrated Earth-system science: a roadmap for 2016-2025
}

Article

Accepted Version

Creative Commons: Attribution-Noncommercial-No Derivative Works 4.0

Simmons, A., Fellous, J.-L., Ramaswamy, V., Trenberth, K., Asrar, G., Balmaseda, M., Burrows, J. P., Ciais, P., Drinkwater, M., Friedlingstein, P., Gobron, N., Guilyardi, E., Halpern, D., Heimann, M., Johannessen, J., Levelt, P. F., Lopez-Baeza, E., Penner, J., Scholes, R. and Shepherd, T. (2016) Observation and integrated Earth-system science: a roadmap for 20162025. Advances in Space Research, 57 (10). pp. 2037-2103. ISSN 0273-1177 doi: https://doi.org/10.1016/j.asr.2016.03.008 Available at https://centaur.reading.ac.uk/65846/

It is advisable to refer to the publisher's version if you intend to cite from the work. See Guidance on citing.

To link to this article DOI: http://dx.doi.org/10.1016/j.asr.2016.03.008

Publisher: Elsevier

All outputs in CentAUR are protected by Intellectual Property Rights law, including copyright law. Copyright and IPR is retained by the creators or other copyright holders. Terms and conditions for use of this material are defined in the End User Agreement. 


\section{CentAUR}

Central Archive at the University of Reading

Reading's research outputs online 


\title{
Observation and Integrated Earth-system Science:
}

\section{A roadmap for $2016-2025$}

\author{
Adrian Simmons ${ }^{1}$ \\ European Centre for Medium-Range Weather Forecasts, Shinfield Park, Reading RG2 9AX, UK \\ Jean-Louis Fellous \\ Committee on Space Research, c/o CNES, 2 place Maurice Quentin, 75039 Paris Cedex 01, France
}

Venkatachalam Ramaswamy

Geophysical Fluid Dynamics Laboratory/NOAA, Princeton, NJ 08540-6649, USA

Kevin Trenberth

National Center for Atmospheric Research, P.O. Box 3000, Boulder, CO 80307, USA

and fellow contributors from a Study Team of the Committee on Space Research: Ghassem Asrar (Univ. of Maryland), Magdalena Balmaseda (ECMWF), John P. Burrows (Univ. of Bremen), Philippe

Ciais (IPSL/LSCE), Mark Drinkwater (ESA/ESTEC), Pierre Friedlingstein (Univ. of Exeter), Nadine Gobron (EC/JRC), Eric Guilyardi (IPSL/LOCEAN), David Halpern (NASA/JPL), Martin Heimann (MPI for Biogeochemistry), Johnny Johannessen (NERSC), Pieternel F. Levelt (KNMI and Univ. of Technology Delft), Ernesto Lopez-Baeza (Univ. of Valencia), Joyce Penner (Univ. of Michigan), Robert Scholes (Univ. of the Witwatersrand) and Ted Shepherd (Univ. of Reading).

\begin{abstract}
This report is the response to a request by the Committee on Space Research of the International Council for Science to prepare a roadmap on observation and integrated Earth-system science for the coming ten years. Its focus is on the combined use of observations and modelling to address the functioning, predictability and projected evolution of interacting components of the Earth system on timescales out to a century or so. It discusses how observations support integrated Earth-system science and its applications, and identifies planned enhancements to the contributing observing systems and other requirements for observations and their processing. All types of observation are considered, but emphasis is placed on those made from space.

The origins and development of the integrated view of the Earth system are outlined, noting the interactions between the main components that lead to requirements for integrated science and modelling, and for the observations that guide and support them. What constitutes an Earth-system model is discussed. Summaries are given of key cycles within the Earth system.
\end{abstract}

\footnotetext{
${ }^{1}$ Corresponding author; email: adrian.simmons@ecmwf.int; tel: +44 1189499700
} 
The nature of Earth observation and the arrangements for international coordination essential for effective operation of global observing systems are introduced. Instances are given of present types of observation, what is already on the roadmap for 2016-2025 and some of the issues to be faced. Observations that are organized on a systematic basis and observations that are made for process understanding and model development, or other research or demonstration purposes, are covered. Specific accounts are given for many of the variables of the Earth system.

The current status and prospects for Earth-system modelling are summarized. The evolution towards applying Earth-system models for environmental monitoring and prediction as well as for climate simulation and projection is outlined. General aspects of the improvement of models, whether through refining the representations of processes that are already incorporated or through adding new processes or components, are discussed. Some important elements of Earth-system models are considered more fully.

Data assimilation is discussed not only because it uses observations and models to generate datasets for monitoring the Earth system and for initiating and evaluating predictions, in particular through reanalysis, but also because of the feedback it provides on the quality of both the observations and the models employed. Inverse methods for surface-flux or model-parameter estimation are also covered. Reviews are given of the way observations and the processed datasets based on them are used for evaluating models, and of the combined use of observations and models for monitoring and interpreting the behaviour of the Earth system and for predicting and projecting its future.

A set of concluding discussions covers general developmental needs, requirements for continuity of space-based observing systems, further long-term requirements for observations and other data, technological advances and data challenges, and the importance of enhanced international cooperation. 


\section{Contents}



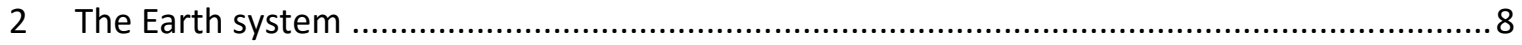

2.1 Development of the Earth-system view …................................................................ 8

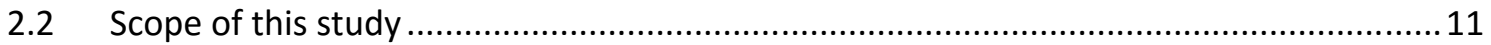

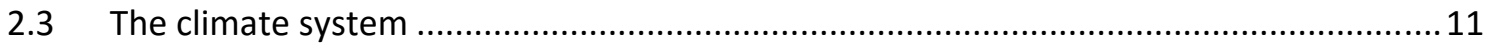

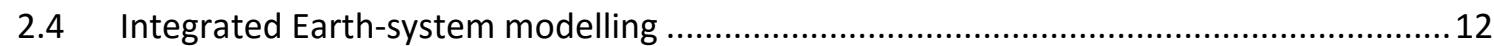

2.5 Energy, circulation and constituent cycles within the Earth system ...............................13

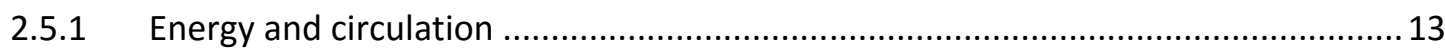

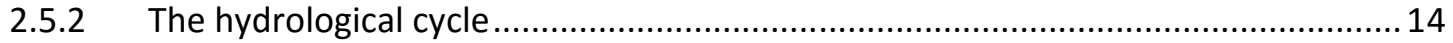



2.5.4 Other constituent cycles and the link between air quality and climate ................... 18

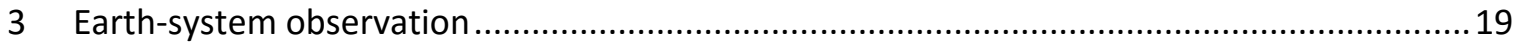

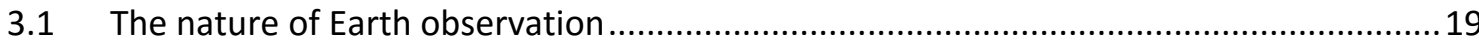



3.3 Sustained satellite observing systems for weather and climate ...................................23

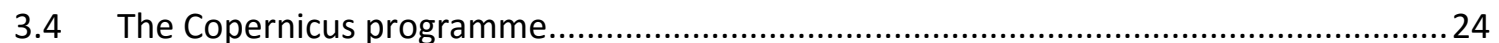

3.5 Missions for research and development, and the challenges of continuity.....................25

3.6 Observation of specific components of the Earth system .............................................28



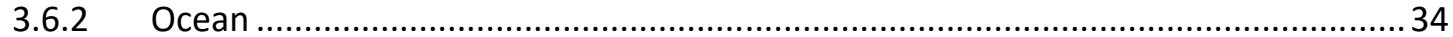

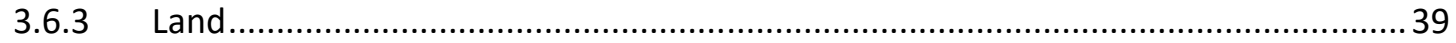



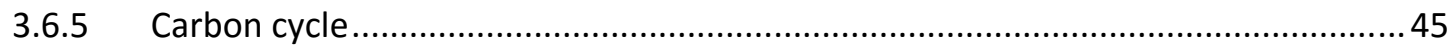

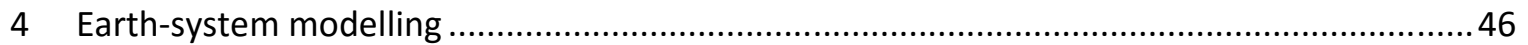

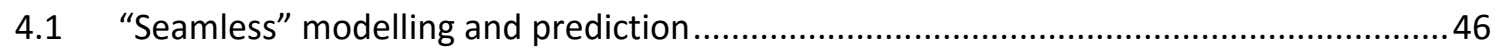

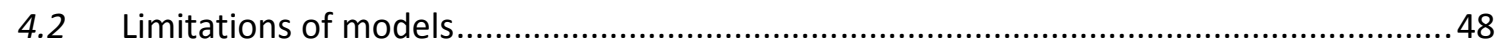

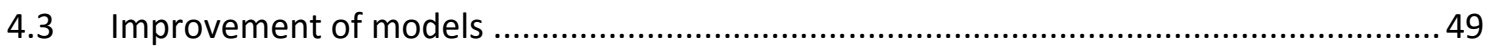

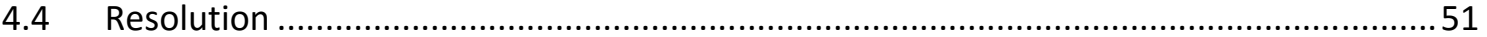







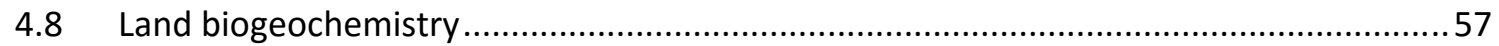

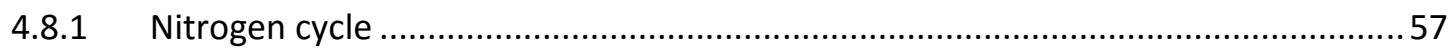




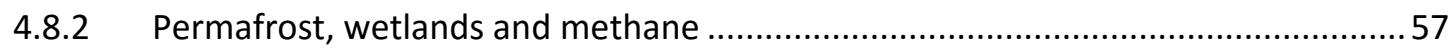



4.8.4 Land use, land management and land cover change .........................................58

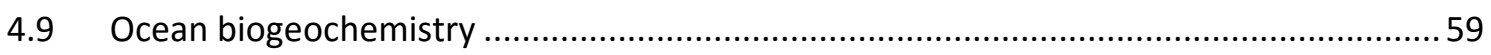

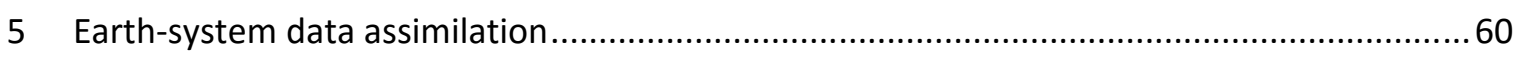

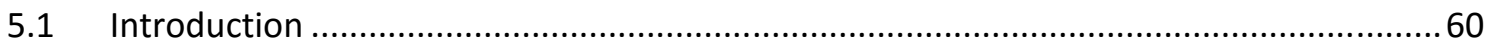

5.2 Data assimilation for specific components of the Earth system......................................61 61



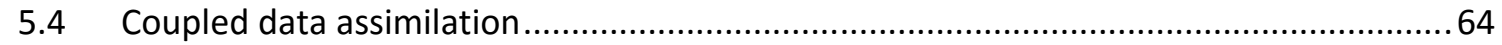

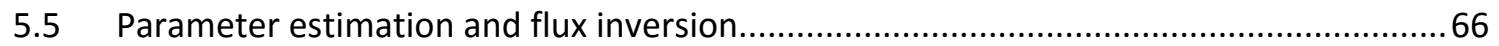

6 Use of observations in integrated Earth-system modelling and its applications .....................68



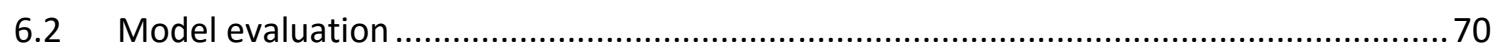



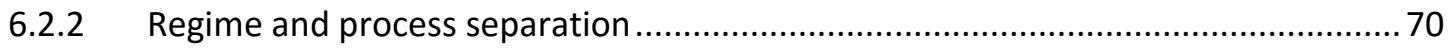

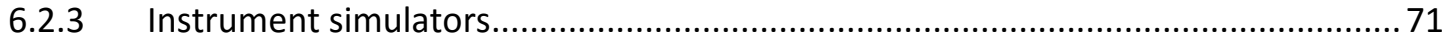

6.2.4 Evaluation at component and Earth-system levels ................................................. 72

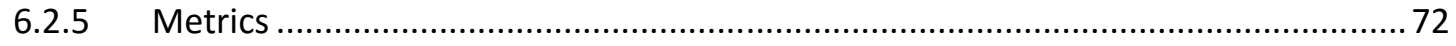

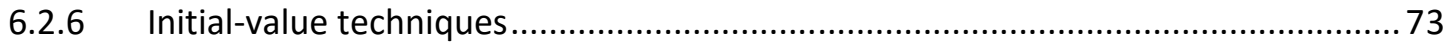

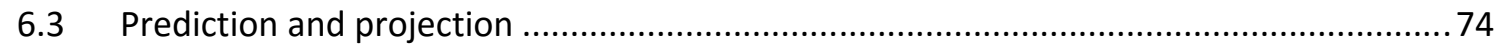

6.3.1 General roles and requirements for observations............................................... 74

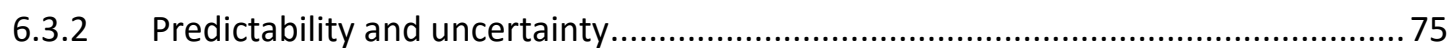

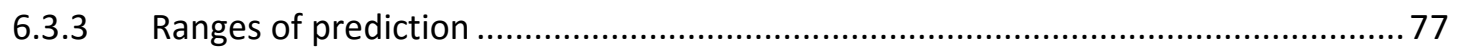

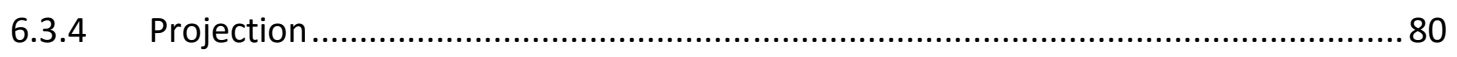



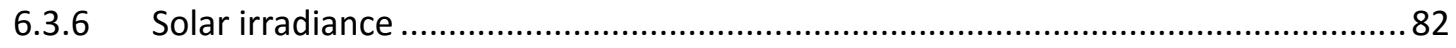

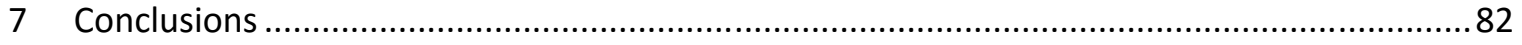

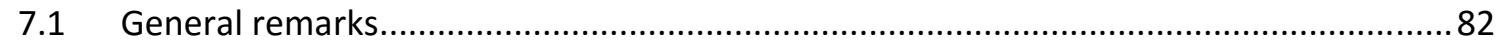

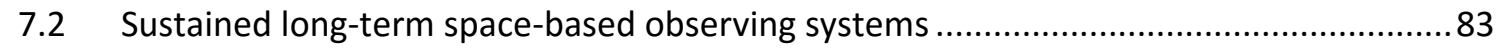

7.3 Other requirements for long-term space-based observations ....................................... 83

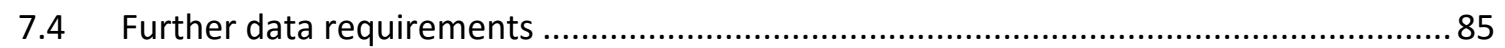

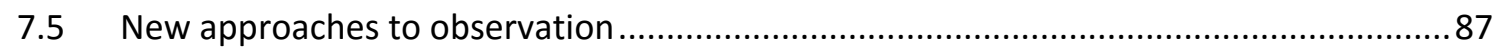

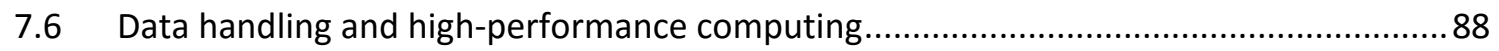

7.7 Enhancement of international co-operation .............................................................. 88 


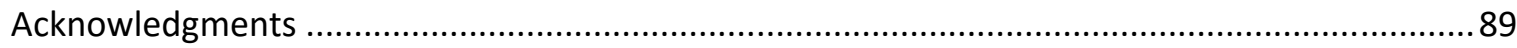

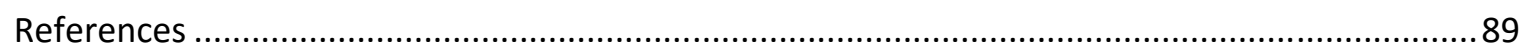

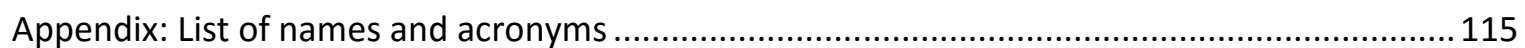




\section{Introduction}

The needs for observation, modelling and understanding of the integrated Earth system are introduced. The origin and purpose of this roadmap report are described, and an outline of the structure of the report is given.

The natural variability of the Earth's atmosphere, oceans and land and ice surfaces has shaped the evolution of life in general and human systems in particular. The impact of that variability continues today. In turn, human activities continue to change the environment in many ways, while that environment has to support the lives of an increasing population. Much of the land surface is no longer in its natural state, and the chemical composition of the atmosphere and ocean is being changed in ways that change climate and affect life. Present activities may leave their mark on the state of the environment for millennia into the future. It has become imperative to monitor, understand and where possible predict many aspects of environmental variability and change.

This requires that observations of sufficient quality, quantity and regularity be made of the key variables of what has come to be called the Earth system. It also requires a capability to model with sufficient realism how these key Earth-system variables are likely to change in the future, whether it be tomorrow's weather or air quality, the prospects for agriculture for the season ahead or projected longer-term changes in climate and their impacts. The development of both observing systems and models in turn requires scientific understanding of the physical and biogeochemical processes at play in the atmospheric, oceanic and terrestrial components of the Earth system, including their interactions with each other and how they influence or are influenced by human activities. Understanding is also needed to evaluate and interpret the results of monitoring, forecasting and the projections that are based on prescribed scenarios for uncertain factors causing environmental change, notably future anthropogenic emissions of key gases and aerosols, and future land use. This in turn provides the basis for sound formulation and communication of essential messages to policymakers, service providers and the general public.

A variety of national and international institutional arrangements are in place to help ensure that the needs for observation, modelling and understanding are met. Among them, the international Group on Earth Observations (GEO) was set up a little over ten years ago to foster new projects and coordinated activities across the full range of Earth observation and to provide the framework for a Global Earth Observation System of Systems (GEOSS) that supplements the arrangements under which its contributing and largely pre-existing observing systems operate. As a contribution to the forward look that the GEO was taking for the second decade of the GEOSS, the Committee on Space Research (COSPAR) of the International Council of Science made a well-received offer to develop a roadmap for observation and the associated modelling of the integrated Earth system, focussed on the coming ten years.

Accordingly, a study team was set up in 2013. The charge by COSPAR to the team was to produce a roadmap report that would:

- foster interdisciplinary scientific research making combined use of modelling and the diverse observations provided by the various systems that contribute to the GEOSS;

- address the functioning, predictability and long-term evolution of the Earth system; 
- discuss how the GEOSS supports integrated Earth-system science and its applications; and

- identify the enhancements to observing systems needed to improve prediction of variability on up to decadal timescales, and the modelling of longer-term change.

Such a report was considered timely because of scientific developments that include the substantial expansion during the 1990s and 2000s of capabilities for observing, modelling and analysing components of the Earth system, the increasing use of Earth-system models for climate prediction and projection, and the prospective use of Earth-system modelling and data assimilation for weather and other environmental forecasting and monitoring. It was seen also as timely for institutional reasons beyond that of GEOSS planning. It would contribute to the assessment activities to be undertaken by the Global Climate Observing System (GCOS) programme in the period 20142016. It would also review current status and needs in the contexts of emerging climate and environmental services, including the intergovernmental Global Framework for Climate Services and Europe's Copernicus programme, and research initiatives such as Future Earth. The timing of the report would also enable it to draw on the conclusions and address some of the scientific uncertainties expressed in the Fifth Assessment Report (AR5) of the Intergovernmental Panel on Climate Change (IPCC).

The roadmap report that is presented here is concerned with observations and their use with integrated (or coupled) modelling of the physical and biogeochemical processes operating in the atmosphere, oceans and land, including ice and snow interfaces, on timescales out to a century or so. The aim of the study team was to cover the principal types of observation that are made today or planned for the future, with a view to 2025 and beyond, and to identify gaps and risks in data provision that threaten the quality of services and the integrity and utility of long-term climate records. It was recognized as important also to keep the past in view, since as good a historical description as possible of the state of the Earth system, including its natural variability and past anthropogenic changes, is needed as a baseline for monitoring the system and assessing the capability of models.

The report discusses how a particular observation of a variable may serve many purposes and how there is much in common to be faced in the modelling needed for different applications. No simple set of priorities emerges from the report, although it acknowledges that balances have to be struck between long-term measurement programmes and occasional more ground-breaking investigative missions, and between investments in improving existing components of models and in incorporating additional processes. It also recognizes that both improved observations and improved extraction of information from existing observations have parts to play. It discusses needs for remote sensing from space, for in situ measurement and for land-, sea- and air-based remote sensing, but gives more emphasis to observation from space in view of the particular role of COSPAR. It is not intended to be a comprehensively referenced review, but reference is given to some influential past scientific papers and reports, to recent reviews and to many other papers and reports chosen for illustrative purposes.

The report is structured as follows. The next section outlines the origin and development of the integrated view of the Earth system, placing emphasis on the interactions between the main components that lead to requirements for integrated science and modelling and for the observations that guide and support them. The scope of the present study is defined. Key cycles 
within the Earth system are discussed. Section 3 gives a summary of the nature of Earth observation and of some of the arrangements for international coordination essential for its effective operation. Instances are given of present types of observation and what is already on the roadmap for 20162025. Observations that are organized on a systematic basis and observations that are made for process understanding and model development, or other research or demonstration purposes, are covered. Aspects of observations specific to particular components of the Earth system are discussed.

The current status and prospects for Earth-system modelling are summarized in section 4. The evolution towards applying Earth-system models for environmental monitoring and prediction as well as for climate simulation and projection is outlined, and general issues related to the improvement of models, whether through refining the representations of processes that are already incorporated or through adding new processes or components, are discussed.

Section 5 discusses the integrated use of observations and modelling in the process of data assimilation, not only because it generates datasets for monitoring the Earth system and for initiating and validating predictions, but also because of the feedback it provides on the quality of both the models and the observations used in the process. Related inverse methods for surface-flux or model-parameter estimation are also discussed. The use of observations, either directly or through processed datasets, is discussed in section 6 in the context of applying and improving Earthsystem models. Topics covered are monitoring and interpretation, the evaluation of models, and prediction and scenario-based projection.

Section 7 presents a summary of the principal observational needs discussed in preceding sections, and provides further and concluding discussion.

\section{The Earth system}

The origins and development of the integrated view of the Earth system are outlined, noting the interactions between the main components that lead to requirements for integrated science and modelling, and for the observations that guide and support them. The scope of the present study is defined. Key cycles within the Earth system are discussed.

\subsection{Development of the Earth-system view}

The 1970s was a landmark decade for sustained observation from space and for progress towards a capability for integrated modelling of the Earth system. 1972 in particular saw the launch of the first of what is now known as the Landsat ${ }^{2}$ series of Earth-imaging satellites, of which the latest is the currently operational Landsat 8 . It also saw the launch of NOAA-2, the first of an unbroken series of operational satellites carrying instruments that provide soundings of atmospheric temperature and humidity. A constellation of geostationary meteorological satellites and the associated international coordination (section 3.2) were also established in this decade and have been sustained ever since. Global observations and models, and their combined use in data assimilation, were the focus of the Global Atmospheric Research Programme (GARP) during the decade, which also saw global systems for numerical weather prediction become operational in the United States of America and Europe. A

\footnotetext{
${ }^{2}$ Satellite names and acronyms are listed in an Appendix
} 
report of the US Committee for GARP (NRC, 1975) offered a view of the climate system and processes responsible for change; the amended form presented by Gates (1979) to the first World Climate Conference (Figure 1) falls not far short of what would today qualify as an Earth-system model (section 2.4, Figure 2). The first atmospheric circulation modelling of the impact of increased carbon dioxide was published by Manabe and Wetherald in 1975. The same year also saw the first publications of results from coupled atmosphere-ocean models (Bryan et al., 1975; Manabe et al., 1975). A report by Kellogg (1977) on the effects of human activities on global climate identified needs for research that continue today: on modelling, quantification of the sources and sinks of carbon dioxide, the response of sea ice and ice sheets to warming, regional changes in temperature and precipitation, and the effects of anthropogenic aerosols and of changes in land use and stratospheric composition.

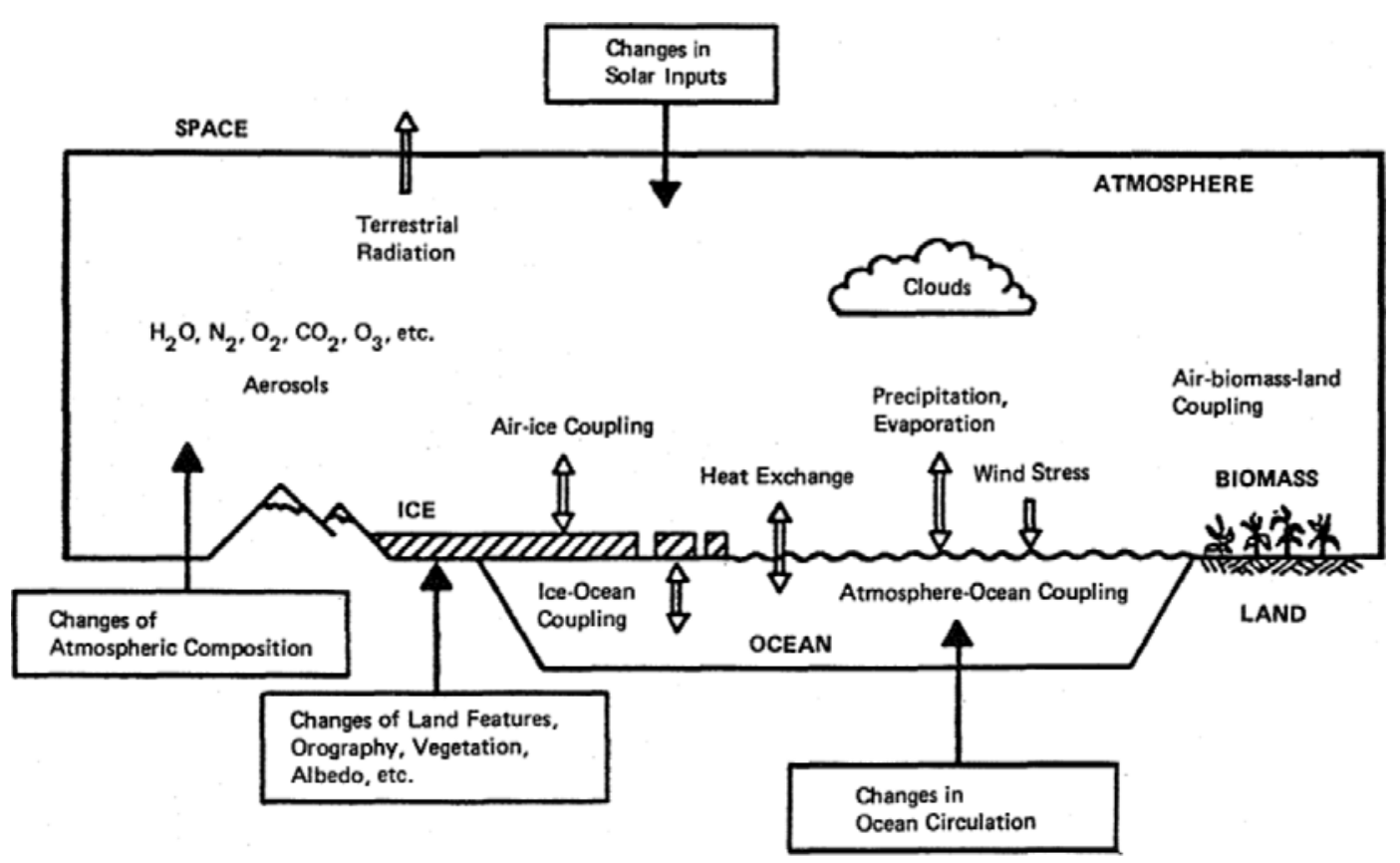

Figure 1 Schematic illustration of the Earth's climatic system, with some examples of the processes responsible for climate and its change. Source: Gates (1979), adapted from NRC (1975).

The Earth-system view took shape in the 1980s. An important step towards it was a 1982 US National Aeronautics and Space Administration (NASA) report entitled "Global Change: Impacts on Habitability". Prepared under the chairmanship of Richard Goody, it proposed "a major NASA research initiative to document, to understand, and if possible, to predict long-term (5-50 years) global changes that can affect the habitability of the Earth." The envisaged programme would involve studies of the atmosphere, oceans and land, including the cryosphere and biosphere, recognising that on "decadal time scales, these regimes and the cycles of physical and chemical entities through them are coupled into a single interlocking system."

A broader view, of the Earth as an interacting system of components including solid-Earth processes, requiring observations and modelling to disentangle interactions and establish causal relationships, was espoused in a 1986 report. Entitled "Earth System Science Overview: A Program for Global Change", it was produced by the NASA Advisory Council's Earth System Sciences Committee, which itself had been set up in 1983 and was led at the time by Francis Bretherton. The report separated the Earth system into the components of the solid Earth, including its core, and the 
components of the fluid and biological Earth. It advocated a division of study by timescale rather than discipline. For study and prediction of global change on a timescale of decades to centuries it was the fluid and biological components that had to be modelled as an interactive system, and for which enhanced observations were required. An observation programme for fundamental solidEarth characteristics was also recommended, but was not the main thrust of the report: the prediction of change over the next decade to century was identified as the challenge to Earth-system science. The NASA Earth Observing System (EOS) programme evolved from this visionary report.

In Europe, the scientific strategy for Earth observation from space developed from the European Space Agency (ESA) "Looking down, looking forward" report (ESA SP-1073, 1985), which offered " $a$ perspective for Earth sciences and applications", leading to the "Living Planet" programme (ESA SP$1227^{3}, 1998$ and SP-1234, 1999). The programme includes science-oriented Earth Explorer missions and an Earth Watch element facilitating delivery of Earth-observation data for use in operational services. The mandate of the complementary European Organization for the Exploitation of Meteorological satellites (EUMETSAT), established in 1986 to run operational meteorological satellite systems, was broadened in 2001 to include contribution to the operational monitoring of climate and detection of global climatic change.

A key component of Europe's current response to a broad range of environmental concerns stems from 1998, when a meeting of national and European space agencies and the European Commission issued a manifesto affirming the importance of observation from space and calling for common European action on global environmental monitoring. By 2001 the main orientation of a Global Monitoring for Environment and Security programme was established. Under the name Copernicus, it is today implementing Earth observation on an operational basis (section 3.4), related atmospheric, land and marine services, and cross-cutting services including one on climate change.

Japan, through its Meteorological Agency (JMA), has contributed with Europe and the USA to the coordinated constellation of geostationary meteorological satellites since the 1970s. Through its Aerospace Exploration Agency (JAXA) and predecessor organizations, Japan has recognized the importance of observation of the wider Earth system and developed a number of important missions, both alone and in collaboration with Europe and the USA. Other nations are playing increasingly important roles. Further discussion is given in section 3 .

In parallel to these observational initiatives, the initial modelling focus on the physical components of the Earth system has been extended to include atmospheric chemistry and the broader biogeochemistry of the land and oceans. Beyond this, the Future Earth initiative (section 3.2) has introduced a broader view of the Earth system that includes interacting human activities within the overall system rather than regarding them as something external that affects or depends on the Earth system.

\footnotetext{
${ }^{3}$ This seminal document devoted to the Earth Science and research element of the Living Planet programme, included a preface by Professor Roger-Maurice Bonnet, then ESA Director of Science, and former President of COSPAR, who initiated the adoption of this programme, which explicitly referred to Earth system models.
} 


\subsection{Scope of this study}

Although there has been considerable progress over the thirty or so years that have elapsed since the "Bretherton report" was published, the observation and integrated modelling and prediction of the Earth system on timescales out to a century or so remains the challenge to Earth-system science that is discussed here. As modelling is becoming more seamless across these timescales (section 4.1), prediction of the Earth system on time ranges from days upward is considered.

Notwithstanding the importance of climate and other environmental change, much of the challenge remains to model well the natural state of the Earth system, to provide a firmer basis for the modelling of change. Observation needs to support the improvement of basic modelling and the initialization of short-term forecasts, as well as detect and contribute to the understanding and prediction of longer-term change.

Although focus is accordingly placed on the observation and modelling of the fluid and biological components of the Earth system, some observation of solid-Earth processes is also relevant on this timescale. This includes monitoring the potential for volcanic eruptions and the vertical movement of land that is a factor in vulnerability to sea-level rise. Little discussion of the modelling of the interacting human component is given as the topic is covered in Future Earth planning reports and integration of substantial human-activity modules in comprehensive Earth-system models is not expected to become commonplace within the ten-year timeframe of this roadmap. Attention also does not extend to the magnetosphere and outer atmosphere, notwithstanding the increasing interest being paid to the monitoring and prediction of "space weather"; this is the subject of a separate COSPAR roadmap study (Schrijver et al., 2015).

\subsection{The climate system}

The glossary of the IPCC's AR5 notes both narrow and wide definitions of climate. Climate in the narrow sense refers to the average weather, or more rigorously a statistical description in terms of the means and variability of weather parameters over a period of interest. The classical averaging period is 30 years, as defined by the World Meteorological Organisation (WMO). The parameters are most often surface variables such as temperature, precipitation and wind. Climate in a wider sense is the state, including statistical properties, of the "climate system".

In turn, the IPCC glossary defines the "climate system" to be "the highly complex system consisting of five major components: the atmosphere, the hydrosphere, the cryosphere, the lithosphere and the biosphere, and the interactions between them. The climate system evolves in time under the influence of its own internal dynamics and because of external forcings such as volcanic eruptions, solar variations and anthropogenic forcings such as the changing composition of the atmosphere and land use change."

In this report the word "climate" is used in the broader sense. The Earth system as considered for the most part here is indistinguishable from the climate system as defined by the IPCC. The report nevertheless addresses the analysis and prediction of the instantaneous state of the system, weather in its most general sense, in addition to being concerned with the statistical characteristics of the system on climate timescales out to century or so. 


\subsection{Integrated Earth-system modelling}

Although the IPCC does not offer an AR5 definition of the Earth system separate to that of the climate system, a specific definition of an "Earth System Model" is provided. The term is used to describe a "coupled atmosphere-ocean general circulation model in which a representation of the carbon cycle is included, allowing for interactive calculation of atmospheric $\mathrm{CO}_{2}$ or compatible emissions. Additional components (e.g., atmospheric chemistry, ice sheets, dynamic vegetation, nitrogen cycle, but also urban or crop models) may be included." In opening the AR5 chapter on the evaluation of climate models, Flato et al. (2013) note that these models "have continued to be developed and improved since the [Fourth Assessment Report], and many models have been extended into Earth System models by including the representation of biogeochemical cycles important to climate change." The complexity and completeness of the representation of the carbon cycle varies among these models, however, particularly in respect of their treatments of land surface processes and ocean biogeochemistry.

In the above view it is the inclusion of the biogeochemical carbon cycle that distinguishes an Earth-system model from the physical climate model provided by coupling models of the atmosphere, ocean, land and ice, in which concentrations of carbon dioxide and other greenhouse gases have to be prescribed in order to study past or projected future climate change. A more general definition is adopted here. The view is taken that for short-term prediction it is the inclusion of faster-acting biogeochemical processes, such as related to air quality and stratospheric ozone depletion, rather than the carbon cycle that distinguishes an Earth-system model from a model of the physical climate system. Furthermore, the faster-acting biogeochemical processes also play roles in the longer-term evolution of the Earth system, through interactions between air quality and climate for example, while modelling the short-term behaviour of long-lived greenhouse gases is important for estimating and understanding the temporal variability and geographical distribution of their sources and sinks. What matters is not some narrow distinction between what is or is not fit to be termed an Earth-system model, but rather that the set of processes included in modelling the Earth system for a particular purpose is appropriate for what is being predicted or simulated, whatever the timescale.

A contemporary illustration of the variables and processes that may be taken into account in Earth-system models is presented as Figure 2. Much of what is depicted was indeed identified when Figure 1 was drawn up some forty years ago, and includes many of the processes fundamental to the working of the natural climate system that need to be represented well in a free-running model, or constrained well by data assimilation in a model used for reanalysis or short-term forecasting. This is necessary for correct functioning of additional processes included within models and correct representation of what are treated as external drivers and impacts. Figure 2 differs from Figure 1 through its addition of ocean biogeochemistry, ecosystems (although Figure 1 has biomass) and the human activities and constructs that are affected by weather and climate, and that contribute to climate and other environmental change.

Earth-system models are needed not only to simulate and understand the Earth system, and to predict its future evolution, but also to help develop an integrated observational picture of the system. Measurements of Earth-system quantities are in many cases indirect, and often have to be pieced together from different sources, with different coverage and accuracies, to construct a global, long-term representation. Models can play a key role in this. Integrated use of observations 
and modelling also provides feedback on the quality of both, and guides the design of observing systems and the development of models. Further discussion is given in several later sections.

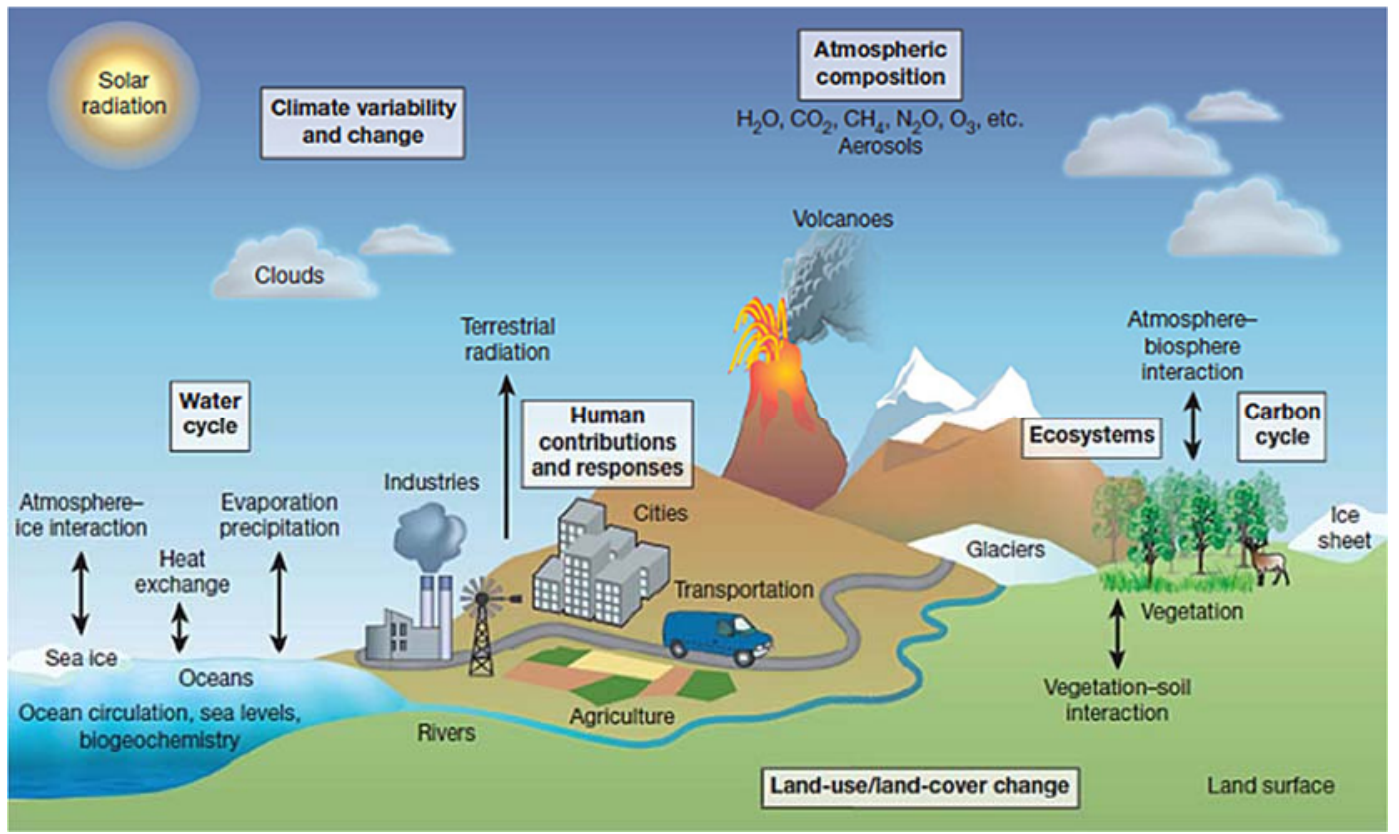

Figure 2 Schematic view of much of what needs to be considered in an Earth System Model. Atmospheric circulation, weather systems, the trace gases important for air quality and secondary aerosol formation, and ocean surface waves also have to be taken into account. Source: www.gfdl.noaa.gov; adapted from Trenberth et al. (1995)

\subsection{Energy, circulation and constituent cycles within the Earth system}

Elements of the functioning of the Earth system may be studied from the viewpoints of the budgets of quantities such as energy or sea-level, the evolution of particular variables or indices of climate change, or the behaviour of a particular domain such as the cryosphere. Studies may also be framed in terms of the cycling of water or chemical elements through the Earth system. Specific attention is paid in this report to the hydrological and carbon cycles, in view of the fundamental importance of water to life and the central and interactive role the cycling of water plays within the Earth system, and in view of the widespread consequences of rising levels of carbon dioxide and methane in the atmosphere.

\subsubsection{Energy and circulation}

The Earth system is driven by the energy received from the Sun. Observations from satellites and at the Earth's surface of incoming and outgoing radiation, observations of the variables related to heat content and estimates from reanalysis are each used to quantify the energy flows and storage within the system. Depictions of the global energy budget of the atmosphere include those by Trenberth et al. (2009), Berrisford et al. (2011) and Wild et al. (2013).

The latest measurements indicate that the incoming solar radiative flux of energy at the top of the atmosphere is $340-341 \mathrm{Wm}^{-2}$ when averaged over the surface of the Earth. Of this some 100 $\mathrm{Wm}^{-2}$ are reflected back to space by clouds and the Earth's surface. The net solar input is almost balanced by thermal emission to space, but a small residual build-up of energy at a rate of 0.5-1 Wm

${ }^{2}$ is inferred from the way the system is changing. AR5 concluded with high confidence that more than $90 \%$ of the energy accumulated by the climate system over the period 1971-2010 has warmed 
the oceans, with much of the rest accounted for by warmer land and melted ice rather than the rise in temperature and moisture content of the atmosphere (Rhein et al., 2013). The atmosphere is nevertheless a significant actor in the system despite its low thermal capacity. It plays a predominant role in the poleward transfer of heat and holds the increasing concentrations of greenhouse gases widely accepted to be the principal cause of the residual warming of the system as a whole.

The atmosphere absorbs solar energy at a rate of about $80 \mathrm{Wm}^{-2}$ and the net solar radiative flux into the Earth's surface is about $160 \mathrm{Wm}^{-2}$. A little more than half of the latter is counterbalanced by the surface latent heat flux associated with evaporation, which exceeds the surface sensible heat flux by a factor of four or more. Net thermal emission from the surface accounts for almost all the remainder. Subsequent transfer of latent heat within the atmosphere, the net warming from release of this latent heat by condensation, especially in tropical convective systems, and the influence of water vapour and cloud on both solar and thermal radiative fluxes link the atmospheric component of the hydrological cycle intimately with the energy balance of the atmosphere.

Geographical variations in heating and cooling can persist only if there are associated circulation systems. Most basic are the mid-latitude westerly jets that are linked with the predominance of solar-induced atmospheric heating in the tropics and sub-tropics, and of radiative cooling at higher latitudes. The synoptic mid-latitude weather systems that develop through the dynamical instability of the westerly flows bring about not only essential thermally balancing poleward transfers of heat but also poleward transfers of momentum that balance frictional losses associated with surface winds that tend to be westerly in middle latitudes and easterly in the tropics. Poleward energy fluxes in the atmosphere are estimated robustly from reanalyses to peak at some $5 \mathrm{PW}$ in the annual average at around $40^{\circ}$ latitude in both hemispheres (Trenberth and Caron, 2001; Fasullo and Trenberth, 2008; Berrisford et al., 2011). Net oceanic poleward transport is much smaller than atmospheric transport at sub-tropical and higher latitudes, but attains comparable magnitude in the tropics, peaking at 1-2 PW at latitudes poleward of $10^{\circ}$.

Considerable complexity underlies the basic picture. The heating of the tropical and sub-tropical atmosphere is far from uniform, geographically or seasonally. The associated Hadley, Walker and monsoonal circulations owe their existence to differential heating, yet influence that heating through their effects on moisture transport and the formation of cloud and rainfall. Evaporation from the warm ocean surface and subsequent latent-heat release are fundamental to the nature of tropical cyclones. Mid-latitude weather systems are modified by latent heat release and organized in storm tracks. The low frequency variability of the atmosphere is influenced by fluctuations in the rate at which energy absorbed by the oceans is released into the atmosphere, which in turn are influenced by the atmospheric forcing of oceanic circulations. Bony et al. (2015) discuss the resulting challenge to research posed by deficient understanding and modelling of the interacting processes involved.

\subsubsection{The hydrological cycle}

The hydrological cycle and its changes over time are of vital societal importance because of the vulnerability of life to shortages, excesses and poor quality of water. Extreme challenges for the sustainable management of fresh-water resources will have to be faced during the $21^{\text {st }}$ century, as will the threats of continuing rises in sea level and of increasingly severe weather events. Changes in the hydrological cycle influence the terrestrial carbon cycle, in which water-use efficiency (the ratio 
of photosynthesis to evapotranspiration) in general rises as the carbon dioxide content of the atmosphere grows, but in which also the behaviour of vegetation changes as rainfall changes. Important short-term forecasting applications include those related to river and reservoir control, availability of power from hydroelectric sources and management of the risks associated with floods and droughts. How such aspects of the hydrological cycle might change in future decades is a fundamental question being addressed by integrated Earth-system science.

Water-related variables include precipitation, soil moisture, streamflow, lake and reservoir levels, snow cover, glaciers and ice, evaporation and transpiration, groundwater, water quality and water use. Improving integrated water resource management requires the bringing together of observations, analyses, models and predictions for these variables within decision-support systems. Better linkages to other relevant weather, climate and socio-economic data are also required. Networks for in situ measurement and the automation of data collection are being consolidated, and the capacity to collect and use hydrological observations is being expanded to places where it is lacking. Much more remains to be done, however. This includes continuing efforts to improve the international exchange of in situ data, which is far from adequate for many of the key variables. The increasing role played by space-based observation, which itself brings requirements for in situ data for calibration and validation, is discussed in several places later in this report.

Understanding and the related capability to model the spatial patterns and temporal characteristics of precipitation, including its diurnal variation, frequency and intensity as well as overall amount, are far from perfect. There are greater than $20 \%$ differences among the global-mean precipitation amounts in the archive of results from the coordinated running of climate models in the fifth phase of the Coupled Model Intercomparison Project (CMIP5; Taylor et al., 2012), and the inter-model spread in regional amounts of precipitation is much greater still. Objective verification of precipitation forecasts from global weather-prediction systems (e.g. Haiden et al., 2012) shows variations in skill between systems that are generally larger than those for temperature and wind forecasts. Performance is typically much poorer in the tropics than in the extratropics, and poorer in summer than winter in the extratropics, pointing to the particular challenges of modelling convection. Modelling the hydrological cycle in general places demands on both the resolution of models and their physical parameterizations, and there are related difficulties to be faced in assimilating observational information.

The GEOSS Water Strategy gives priority to the use of water-related Earth observations in six critical theme areas, namely enhancing the global security of domestic and useable water supplies, adapting water resource systems to the impacts of climate change, meeting the water-related health and welfare needs of the poor, protecting from hydrometeorological extremes such as floods and droughts, ensuring access to water for ecosystems and biological systems, and addressing the more general water-food-energy security nexus that results from growing populations, growing consumption as countries develop, and climate change (World Economic Forum, 2011). The latter theme requires development of integrated datasets, analyses and management tools for agriculture and the generation and use of energy. Increasing food costs driven by extreme events and increasing demands for feedstock by the biofuel industry lead to increased demands for both water and energy. Food production relies on both water and energy inputs, especially in areas with widespread irrigation. Energy production has its own water requirements, as water is both a component of the production process and a means for removing heat and waste products from various energy 
production systems. Given the anticipated growth of water use in the food and energy sectors, new ways to increase its efficiency are needed (Lawford, 2014).

\subsubsection{The carbon cycle}

The global carbon cycle as considered here describes the cycling of carbon through the atmosphere, ocean and terrestrial biosphere. Only on timescales longer than 10,000 years do carbon exchanges with the lithosphere through volcanism and erosion of rock also have to be taken into account. Emissions of carbon dioxide caused by humans have led to a $40 \%$ increase in its atmospheric concentration since pre-industrial times, more than half of which has been since 1975 . It is noted in AR5 (Ciais et al., 2013) that emissions from the burning of fossil fuels increased more rapidly during the last decade than during the previous one; Le Quéré et al. (2014) estimate that emissions in 2012 were $9.7 \pm 0.5 \mathrm{PgC} \mathrm{yr}^{-1}$, around 60\% larger than in 1990. This large increase is in stark contrast to the ambition of the Kyoto Protocol, where a number of industrialized countries agreed to maintain their emissions at close to the 1990 level for the period 2008-2012.

Emissions from land-use change are the second human cause of the observed increase in carbon dioxide. These emissions rose steadily from 1850 until the mid-1990s, after which they appear to have first decreased and then remained stable. Deforestation is mainly occurring in a few tropical countries with large forest areas. The global magnitude of land-use-change emissions was only $10 \%$ of that of fossil-fuel emissions in 2012 (at $1 \pm 0.5 \mathrm{PgC} \mathrm{yr}^{-1}$ ), but was comparable in magnitude to fossil-fuel emissions during the first half of the $20^{\text {th }}$ century.

Most of the increase in fossil-fuel emissions of carbon dioxide over the last decade has taken place in emerging economies, where uncertainties of emission inventories are larger than in countries that have been industrialized for much longer. Consequently, the uncertainty of global fossil-fuel emissions has increased faster than the emissions themselves, and is now as large in absolute value as that of half of the emissions from land-use change. This is a concern, because if the dominant human perturbation of the global carbon cycle has an uncertainty that increases with time, the ocean and land sink uncertainty deduced from emissions will inevitably increase.

The global budget of anthropogenic carbon, obtained by taking the difference between the sum of fossil-fuel and land-use-change emissions and the accurately observed yearly increase of carbon dioxide in the atmosphere, reveals that since 1958, when the Mauna Loa record of atmospheric concentration began, on average about half the carbon dioxide emitted into the atmosphere has been absorbed each year by natural oceanic and terrestrial carbon reservoirs. The current estimate of the ocean sink given by Le Quéré et al. (2014) is $2.9 \pm 0.5 \mathrm{PgC} \mathrm{yr}^{-1}$, and the corresponding land sink is $2.7 \pm 0.9 \mathrm{PgC} \mathrm{yr}^{-1}$. In the global budget of anthropogenic carbon, the land sink is deduced from the emissions, the observed atmospheric increase and the ocean sink. The latter is obtained from ocean models constrained by ocean observations. The terrestrial sink (the increase of carbon through time in the terrestrial reservoir) cannot be measured directly and is not strongly constrained by observations, although measurements from forest inventories, for which there are many samples from the northern extratropics but very few from the Tropics, do enable a net increase of carbon in forest biomass worldwide to be inferred (Pan et al. 2011). Changes in soil carbon storage can be measured locally, but their assessment on regional scales requires the use of models. The terrestrial sink, unlike the ocean sink, is highly variable from year to year, and most of its variability can be 
explained by the forcing from El Niño/La Niña variability and cooling following large volcanic eruptions.

Methane is a potent greenhouse gas whose increase accounts for a significant fraction of the radiative forcing of climate change, despite having a much lower atmospheric concentration than carbon dioxide. Its lifetime is controlled by removal through chemical reactions with hydroxyl radicals, and at around a decade is very much less than the lifetime of carbon dioxide. Emissions of methane are significant not only because of the direct radiative effect of the gas itself, but also because changes in methane lead, through oxidation, to changes in ozone and stratospheric water vapour, and their radiative effects. AR5 (Figure TS.7 of Stocker et al., 2013; reproduced here as Figure 3) indicates that the net radiative forcing of change due to methane emissions may exceed half that due to emissions of carbon dioxide. AR5 also records that the concentration of methane has increased by a factor of 2.5 since preindustrial times, with very high confidence that the increase has been caused by mankind, mostly through the growth of emissions from livestock farming, ricepaddy agriculture, fossil fuel extraction and use, and landfills and waste. The causes of the pronounced variability in methane growth that has been observed in recent decades are a matter for continuing debate (Ciais et al., 2013; Kirschke et al., 2013).



Figure 3 Radiative forcing $\left(\mathrm{Wm}^{-2}\right)$ of tropospheric climate change during the industrial era partitioned according to emitted compounds. The two sets of tabulated values in the right-hand columns differ due to differing extents to which conditions are held fixed in making the calculations of the changes in net radiative fluxes that result from changes in emissions over the era. Source: Stocker et al. (2013; Fig. TS.7), where further details may be found. 
Changes in biogeochemical systems in general pose a particular challenge for global observation. The sources and sinks of key species may be inferred from observations of atmospheric concentrations using inverse methods, as discussed later in this report, but may alternatively be estimated using diagnostic surface modelling together with observations of variables such as aboveground biomass, the greenness of vegetation, ocean colour and wetland extent. Furthermore, the limited basic knowledge of some of the important longer term biogeochemical processes (associated with permafrost degradation, vegetation dieback and nutrient limitation effects, for example) requires that the observing system be able to detect "surprises": it must be robust enough to allow the timely identification of developments that are not represented in current diagnostic surface models. Complication also arises, primarily for terrestrial ecosystems, from the multitude of human management activities (related to forestry, irrigation, harvesting and so on), which fundamentally modify ecosystem behaviour. Addressing this challenge requires an observation strategy that integrates space-based and in situ observations together with statistical socio-economic information in a comprehensive modelling framework.

\subsubsection{Other constituent cycles and the link between air quality and climate}

Figure 3 illustrates the degree to which quantification of the anthropogenic radiative drivers of change in the climate system, and of associated uncertainties, has to take into consideration not only carbon dioxide and methane, but also both other well-mixed (and long-lived) gases, particularly nitrous oxide and the halocarbons, and shorter-lived species including aerosols and ozone ${ }^{4}$. The AR5 chapter by Myhre et al. (2013) provides comprehensive discussion. There is a direct link between climate and air quality through the shorter-lived atmospheric constituents involved. Physically consistent modelling that is aligned with observed behaviour is required for the key constituents, from emissions to transport and transformation to sinks. Monitoring and forecasting air quality as well as the estimation of climate forcing requires measurements not only of the constituents that are directly damaging to health or the environment, or that change radiative forcing, but also of the precursors that through chemical reactions influence the concentrations of the harmful species. Data are needed on constituents that include aerosols, tropospheric ozone, nitrogen and sulphur dioxide, ammonia, formaldehyde and other volatile organic compounds, methane and carbon monoxide. Continuity of measurement and timely provision of associated socio-economic data are important for sustaining operational air-quality forecasting and for ensuring that emission databases are up to date. The need to monitor species and conditions related to stratospheric ozone depletion brings additional observational requirements.

The link between air quality and climate forcing means that legislative policy for control of air quality should not be considered independently of policy for limiting anthropogenic climate change. It has become of pressing importance to improve scientific understanding of the interactions involved, as a basis for taking the most appropriate measures. This is one of the aims of the Climate and Clean Air Coalition (www.ccacoalition.org/), a partnership of a number of countries, intergovernmental organizations and other bodies established with the overall objective of reducing short-lived climate pollutants.

\footnotetext{
${ }^{4}$ Methane is variously described as well mixed, short lived or long lived; each description can be found in the IPCC's Fifth Assessment Report.
} 
Cycles of nutrients are vitally important for the functioning of the Earth system, although their inclusion as variables in Earth-system models is not widespread. This is discussed briefly in section 4.8.1 in the case of nitrogen. AR5 expressed high confidence that low nitrogen availability will limit carbon storage on land (Ciais et al., 2013). The role of phosphorus in limiting terrestrial carbon uptake was considered more uncertain, but could become more important than that of nitrogen on centennial time scales.

\section{Earth-system observation}

A summary is given of the nature of Earth observation and of some of the arrangements for international coordination essential for continuing effective operation of sustainable global observing systems. Instances are given of present types of observation and what is already on the roadmap for 2016-2025. Observations that are organized on a systematic basis and observations that are made for process understanding and model development, or other research or demonstration purposes, are covered. Aspects of observation specific to particular components of the Earth system are discussed.

\subsection{The nature of Earth observation}

Observation of the Earth system relies on a complementary mix of remote sensing and in situ measurement. There are needs for both types of observation, and each has its strengths and weaknesses. Much of the remote sensing is from space, mostly involving downward-looking (nadir) observation, but including measurements from viewing the Earth's limb. It comprises passive sensing of the electromagnetic radiation emitted or reflected by the Earth system in the spectral range from the ultraviolet to the microwave, active sensing of the reflection by the Earth system of radiation emitted from satellites, sensing of the occultation of solar and stellar radiation and of signals from Global Navigation Satellite Systems (GNSSs), and sensing of local variations in mass of the Earth system from variations in the gravity field experienced by satellites.

In addition to in situ measurement of the state of the physical, chemical and biological environment, there is an increasing need also for socio-economic data for estimating and developing the modelling of anthropogenic impacts on the environment, and of the impacts of environmental variability and change on human and other life. This includes the data on population, economic development, energy production and other factors used to estimate past, present and possible future anthropogenic emissions of the greenhouse gases, reactive gases and aerosols that influence climate and air quality.

Satellites provide the global or near-global coverage that is needed to describe the Earth system, but are limited in what they can sense. For the atmosphere this includes limits to the extent to which fine-scale vertical structure can be resolved and to which information can be provided on wind and below clouds. For ocean and land, the information provided from space is largely restricted to the near-surface layer, although important inferences can be drawn on bulk properties from altimetry and gravimetry. In situ data are an essential complement, sampling depths and variables that are beyond the view from space and providing detailed vertical structures and temporal resolution, and longer historical records. They also serve as anchor points that support the calibration and validation of satellite observations and derived data products. In situ data generally have far from uniform geographical coverage, however. Moreover, a multiplicity of national institutional arrangements for 
making the required types of measurement poses serious challenges for overall observing-system management, long-term funding and open international data availability.

Instrumental observations in general are subject to changes over time in coverage, in spatial and temporal resolution, and in biases and other characteristics of error. These changes make monitoring and understanding multi-decadal variability and change a challenge. They lead to activities directed towards reprocessing of datasets to inter-calibrate the data from different instruments or to improve the homogeneity of data in other ways, and to benefit from improved knowledge of instrument characteristics and better methods of retrieval of geophysical data products. They also lead to model-assisted integration of data of various types, using the data assimilation approach established for initialising weather forecasts, in the process known as reanalysis that is discussed in section 5.3. Observational campaigns set up for the calibration and validation of data from satellite missions, and reference sites making long-term measurements of especially high quality, such as the GCOS Reference Upper-Air Network (GRUAN; Seidel et al., 2009; Bodeker et al., 2015) have crucial roles to play in this regard. The GCOS programme also defines baseline networks important both for basic monitoring of the variables concerned and for improving the use of data from comprehensive networks and satellite systems.

Also important is the continued collection of the proxy data that provide the record of how climate has changed over the vast period prior to the era of instrumental observation. This type of data includes many natural proxies such as trees, corals and ice cores, and stretches back as far as tens of millions of years ago in the case of estimates of carbon-dioxide concentrations based on geological evidence. It also includes written histories for the more recent past. Acquiring and modelling such palaeoclimatological data are essential for documenting and interpreting changes in climate through time, for placing the instrumental data record for several variables in a longer-term context, and for comparing the performance of models in their simulations of conditions that differ substantially from those experienced over the past century or so. Plentiful examples are given in AR5 by Masson-Delmotte et al. (2013) and others. Further discussion is, however, beyond the scope of the present study, which places emphasis on the space-based component of Earth observation, in recognition of COSPAR's primary interest.

\subsection{International coordination of Earth observation}

No single nation or region of the world has the capabilities and resources to develop and operate a full Earth observing system, not least because in situ observations are required over national territories, including airspace and coastal ocean zones. This has been recognized by the establishment of various arrangements for the international coordination that is essential for effective provision of the observations needed to support integrated Earth-system science and its applications.

Formal international coordination of weather observation can be dated back to the First International Meteorological Conference in 1853 and the establishment twenty years later of the International Meteorological Organization. Since 1950 it has been undertaken under the auspices of WMO, a specialized agency of the United Nations whose interests today extend to include water, climate and related environmental matters. Coordination of ocean observation falls under the Intergovernmental Oceanographic Commission (IOC) of the United Nations Educational, Scientific 
and Cultural Organization (UNESCO), founded in 1960, which works together with WMO on areas of joint interest.

COSPAR itself was established by the International Council of Scientific Unions (ICSU, now the International Council for Science) in 1958 to promote scientific co-operation in space at a time when the first artificial Earth-orbiting satellites had been launched by the Soviet Union and the USA, and in the light of the successful programme of internationally coordinated observations being undertaken during the International Geophysical Year. Since then, the changing political environment and emergence of additional providers of observations from space has led to new mechanisms for the coordination of activities among the national and intergovernmental agencies that operate space programmes. Arrangements have evolved also for the coordinated expression of the requirements for Earth observation.

The Coordination Group for Meteorological Satellites (CGMS, formerly the Coordinating Group for Geostationary Satellites) came into being in September 1972, when representatives of Europe, Japan and the USA, and observers from WMO and GARP, met to discuss questions of compatibility among geostationary meteorological satellites. The CGMS promotes coordinated operation and use of data and products from its members' satellite systems, in support of operational weather monitoring and forecasting and related aspects of climate monitoring.

The Committee on Earth Observation Satellites (CEOS) was established in 1984 with the broader remit of coordinating international efforts for Earth observation as a whole. Its original focus was on interoperability, common data formats, the intercalibration of instruments and common validation and intercomparison of products. CEOS now also provides an established means of communicating with external organizations to respond to requirements for Earth observations. Important in the present context are collaborative activities with CGMS in developing, together with the WMO Space Programme, a strategy for climate monitoring from space (Dowell et al., 2013) and in establishing a joint working group on climate.

The programme for GCOS (Houghton et al., 2012) has been co-sponsored by WMO, IOC, ICSU and the UN Environment Programme (UNEP) since 1992 to provide a coordinating role for climate observation across the atmospheric, oceanic and terrestrial domains. The programme identifies the inadequacies of climate observation and the actions required to address them. Those actions related to space-based observation have been responded to by CEOS; in this regard both GCOS and CEOS have reported under the UN Framework Convention on Climate Change (UNFCCC). Requirements are formulated largely for a set of Essential Climate Variables (ECVs; Bojinski et al., 2014). Those for datasets on ECVs founded on observation from satellites (GCOS, 2011) have provided the basis for the ESA Climate Change Initiative (CCl; Hollmann et al., 2013).

The World Climate Research Programme (WCRP) also plays a significant role in Earth observation in addition to its fundamental promotion of research into the functioning, modelling and prediction of climate. Following on from GARP in 1980, and under the co-sponsorship of WMO, IOC and ICSU, it has been associated with significant observational programmes. A notable example is the establishment of measurements from an array of moorings in the tropical Pacific Ocean under the 1985-1994 Tropical Ocean Global Atmosphere programme. This now-named TAO/TRITON array continues to this day to provide essential data for the prediction and monitoring of El Niño/La Niña events, notwithstanding recent difficulties (GCOS/GOOS/WCRP, 2014). Another example is its WOCE 
(World Ocean Circulation Experiment) programme, which triggered the establishment of the spacebased component of the Global Ocean Observing System (GOOS), inaugurated in the early 1990s by the European ERS-1 and the US-French Topex/Poseidon missions.

WCRP works with GCOS in several ways, including through a set of expert panels on climate observation, and within its own component projects has important initiatives on assessment of observational datasets and their use in evaluating models. It has also worked with partners such as the ICSU-sponsored International Geosphere-Biosphere Programme (IGBP) through their joint membership of the Earth System Science Partnership. This is being superseded by arrangements with Future Earth, an initiative launched in 2012 by a multi-partner alliance including ICSU, UNEP, UNESCO and WMO. It is not currently envisaged that Future Earth will establish major new infrastructure for Earth observation or gathering socio-economic data, but rather that it will work with existing observing systems and coordinating bodies, communicating new data needs as it identifies them. It nevertheless is inheriting responsibility for projects from pre-existing Earth-system science programmes such as IGBP that include important observational components. Future Earth also has an ambitious vision for 2025 (www.futureearth.org/our-vision) to deliver inter alia a "new generation of integrated Earth system models to deepen our understanding of complex Earth systems and human dynamics across different disciplines".

Established in 2003 with the broadest remit concerning observations, the Group on Earth Observations (GEO) is an ad hoc intergovernmental group of approaching 100 countries and the European Commission that works with around 90 participating international organizations to foster new projects and coordinated activities across the full range of Earth observation. GEO is building the Global Earth Observation System of Systems (GEOSS) to provide a framework for integrated global Earth observation that supplements the arrangements under which contributing pre-existing systems operate. Its activities over its initial ten years of operation were organized into nine societal benefit areas (SBAs) and cross-cutting initiatives. These SBAs included some, among them weather and climate, for which observation and modelling plays a central role, and others such as disasters and health that benefit from observational products. Cross-cutting initiatives include an important emphasis on data sharing. GEO has recently developed a new strategic plan for implementing the GEOSS, to run from 2016 to 2025.

The Global Geodetic Observing System (GGOS; Boucher et al., 2015) was established about the same time as GEO, and is one of GEO's participating organisations. As a component of the International Association of Geodesy, GGOS works to provide the geodetic infrastructure necessary for monitoring the Earth system and for global change research. Its support for altimetric and gravimetric missions is of particular importance in the context of this report.

The GEOSS Carbon (Ciais et al., 2010) and Water (Lawford, 2014) Strategy reports have been drawn on in preparing this roadmap, along with the assessment, strategy and planning documents of the other bodies cited in this section, most particularly the implementation plan and supplemental report detailing requirements from satellites published by $\operatorname{GCOS}(2010,2011)$. Extensive use has also been made of material from several cited chapters of the IPCC'S AR5. This roadmap has been prepared in parallel with GCOS (2015), a status report on the global observation of climate, and shares a few sections of text with that report. The GCOS report provides much more detail on in situ observation than this report, and although the focus of the GCOS report is on climate, much of it 
applies also to observation of shorter term variations of those components of the Earth system that are included within the scope of this COSPAR report.

\subsection{Sustained satellite observing systems for weather and climate}

Routine sustained delivery of data from operational polar-orbiting and geostationary satellite systems is fundamental to the provision of services for weather, climate and other aspects of the Earth system. Today China, the European member states of EUMETSAT, India, Japan, South Korea, Russia and the USA each operate multi-instrumented meteorological satellites addressing a spectrum of needs. Several international agreements cover deployments and data exchange, as illustrated below. These satellites deliver data in near-real time that are suitable for use in numerical weather prediction. Much of the data also make important contributions to the climate data record, and typically benefit from reprocessing activities to recalibrate them, in particular as carried out under the Global Space-based Inter-Calibration System (GSICS) initiated by CGMS and WMO.

Longstanding co-operation has already been noted for geostationary systems. This includes instances of the deployment of a backup geostationary satellite of one operator over the region normally covered by another operator, when needed to avoid gaps. More recently, the USA and Europe have formalized the Joint Polar System (JPS) concept in which responsibilities for the "midmorning" and "afternoon" sun-synchronous polar orbits are divided and shared. Figure 4 shows the US view of its resulting polar-satellite programme, comprising coverage of the "early-morning" orbit by satellites of the US Defense Meteorological Satellite Program (DMSP) as well as the complementary JPS coverage of the mid-morning orbit by first and second-generation European satellites (Metop and Metop-SG), and of the afternoon orbit by US NOAA satellites.

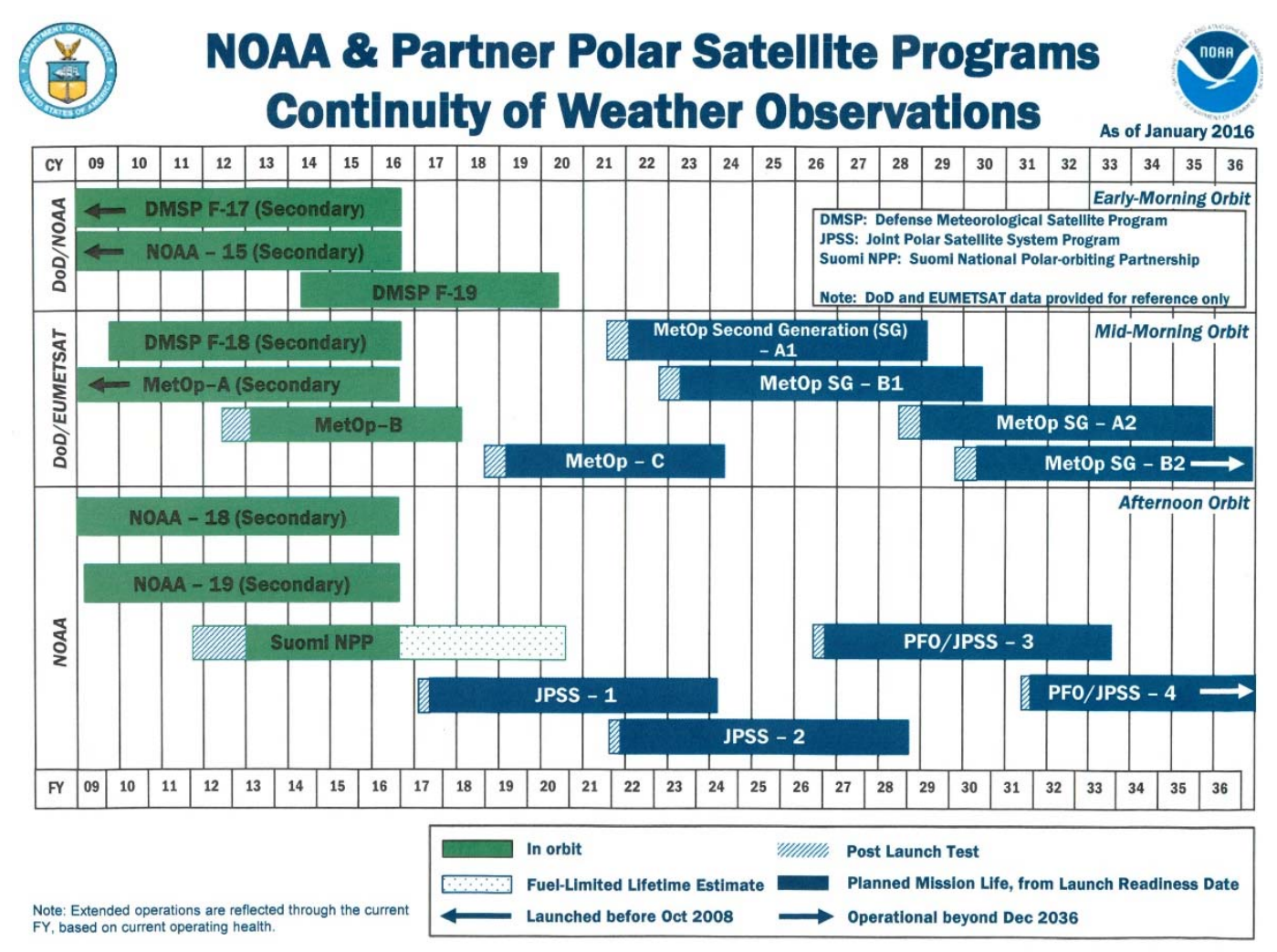

Figure 4 NOAA, EUMETSAT and US Department of Defense (DoD) polar operational satellite programmes as of April 2015 Source: www.nesdis.noaa.gov/flyout_schedules.html 
A further important contributor to the roadmap to 2025 is the series of Chinese FY-3 polarorbiting meteorological satellites. Here CGMS has played a key role through discussion and presentation of the case for complementary coverage of the early-morning orbit by changing the planned deployment of two FY-3 satellites (Eyre and Weston, 2014). FY-3 also provides resilience for other orbits. A bilateral co-operation agreement between EUMETSAT and the China Meteorological Administration includes data and product exchange. The European Centre for Medium-Range Weather Forecasts (ECMWF) started operational assimilation of data from the microwave humidity sounder on the FY-3B satellite in September 2014.

Generation of operational sea-surface-temperature (SST) products makes use of a variety of satellite data, some from the operational polar-orbiting and geostationary meteorological satellites and some from missions that are nominally for research and development (section 3.5). Here too there are collaborative arrangements made both through international coordination mechanisms, for example the International Group On High Resolution SST (GHRSST) and the CEOS "virtual constellation" for SST, and through bi-lateral arrangements, such as that between Japan and the USA for use of all-weather C-band passive microwave data from the AMSR-2 instrument on JAXA's GCOM-W1 satellite, the first in two series of satellites planned to make observations related to the water and carbon cycles.

Operational altimeter data are presently delivered by the Ocean Surface Topography Mission/Jason-2 mission, a joint venture between Europe and the USA, providing continuity beyond Topex/Poseidon and Jason-1. The partnership continues with Jason-3, launched in January 2016. A follow-on Jason Continuity of Service mission (Jason-CS) is planned. Chinese altimeters are now becoming part of the CEOS Virtual Constellation for Ocean Surface Topography.

\subsection{The Copernicus programme}

Copernicus is a major European programme for operational Earth observation and associated service delivery that complements and substantially extends the Earth observation programmes established in support of weather forecasting and related climate monitoring. The launch in April 2014 of Sentinel-1A saw the first spacecraft in orbit out of a series of six so-called Sentinel families (Figure 5 ) that should all be operational well within the timeframe of this roadmap. Sentinel-2A was launched in June 2015 and Sentinel-3A in February 2016. ESA is responsible for developing the Sentinels on behalf of the European Union; operation is being shared with EUMETSAT. Delegated bodies provide products and services. Each Sentinel family is associated with a series of satellites, each replenished as age or health dictates. Copernicus data and products are free and open to access and use. Berger et al. (2012) discuss their potential for addressing some of the challenges associated with advancing Earth-system science.

Sentinel-1 will in due course comprise a pair of C-band SAR satellites (1A and 1B) for operational monitoring, for example of sea ice, land-motion risks, floods and oil spills, and disaster response. Sentinel-2A is a complementary optical imaging satellite that will likewise be subsequently joined in orbit by Sentinel-2B. Sentinels 3 to 5 have different goals, and will use optical imaging sensors, radiometers and spectrometers to measure a wide range of variables. The two Sentinel-3 satellites have the main objective of providing data on sea-surface topography, sea- and land-surface temperature and ocean- and land-surface colour, although they will also provide valuable information on other variables, for the atmosphere also. The Sentinel-4 and -5 instruments will be 
deployed not on dedicated satellites but respectively on the operational meteorological geostationary (Meteosat Third Generation) and polar-orbiting (Metop-SG) platforms, where they will complement other instruments, together providing a rich set of data for monitoring climate and air quality. A dedicated Sentinel-5 precursor satellite flying the TROPOMI instrument, referred to below as Sentinel-5p, will be launched in 2016 to minimise the shortfall in key atmospheric composition data resulting from the loss of Envisat in April 2012, and to complement and extend the observations provided by the OMI instrument on the EOS-Aura satellite. Sentinel- 6 is the Jason-CS mission, and secures operational continuity to the Jason altimetry series of missions within the Copernicus space component through a European-US partnership.

\section{COPERNICUS SENTINELS}

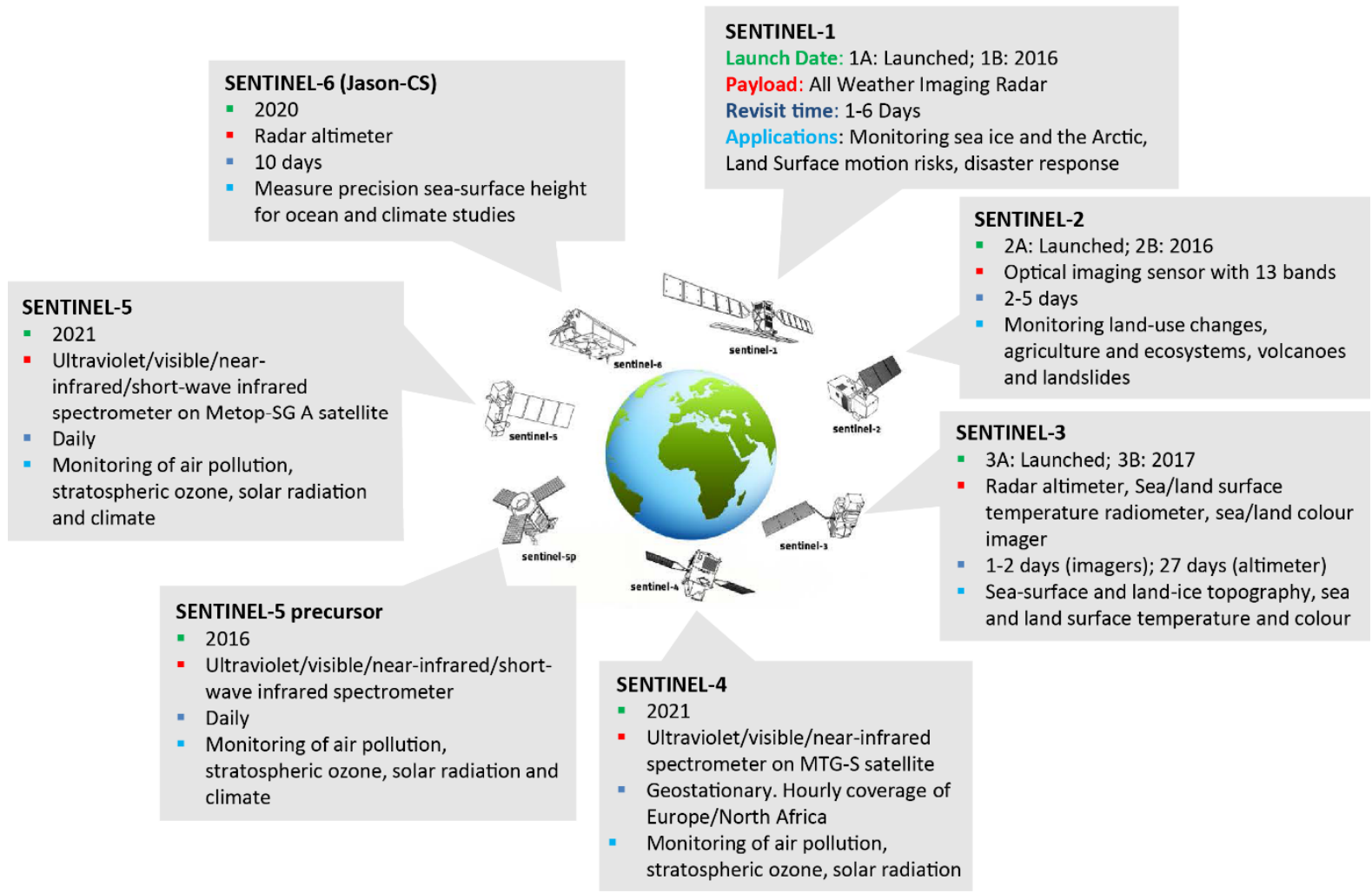

Figure 5 Overview of the satellites of the Copernicus system. Source: M. Drinkwater, adapted from Butler (2014)

\subsection{Missions for research and development, and the challenges of continuity}

Beyond the sustained observations provided by operational programmes such as discussed in the preceding two sections, many space agencies operate time-limited "research" missions for shortterm measurement of quantities not covered by the operational programmes, for understanding processes and enhancing their modelling, or for development and demonstration of new capabilities. Such missions may be developed after being identified by comprehensive assessments of need, such as those of the US National Research Council's decadal surveys (NRC, 2007), or as a result of individual calls for proposals from the scientific community, such as those for "core" and "opportunity" missions issued by ESA's Earth Explorer programme (Drinkwater et al., 2012). Missions are increasingly carried out through the cooperative efforts of more than one agency. They sometimes involve either repeated deployment of a particular type of instrument or the deployment of an instrument similar in type to an earlier one, and may be followed by implementation of this 
type of measurement within operational programmes such as those discussed earlier. They may thus provide part of a much longer time series of critical measurements, and as such may provide data that are used in operational prediction or monitoring systems, with recalibration as needed. Examples are the use of data on ocean surface vector wind provided by scatterometers on the ERS1, ERS-2, QuikSCAT, Metop-A, Oceansat-2, Metop-B and other satellites, on aerosol optical depth provided by the MODIS instruments on the Terra and Aqua EOS satellites and the VIIRS instrument on the Suomi NPP satellite, and on ocean surface-wave height from the radar altimeters on ERS-1, ERS-2, Envisat, Jason-2, CryoSat and SARAL, to be followed by the data from Sentinel-3. For reactive trace gases such as $\mathrm{NO}_{2}, \mathrm{SO}_{2}$ and ozone, continuation and refinement of the types of nadir measurement provided by a mix of research and operational missions flying BUV, SBUV, TOMS, GOME-1, SCIAMACHY, GOME-2, OMI and OMPS instruments will largely come from the operational NOAA (JPSS), Copernicus (Sentinels $4,5 p$ and 5 ) and Chinese (FY-3) missions.

Recent, current and future groups of related missions include those measuring soil moisture and/or ocean surface salinity (SMOS, Aquarius/SAC-D and SMAP), sea-ice thickness (CryoSat and the forthcoming ICESat-2), and clouds, aerosols and radiation (the A-train set comprising CALIPSO, Cloudsat and PARASOL, and the forthcoming EarthCARE). The 3MI instrument to be flown on MetopSG will build on heritage from the POLDER instrument on PARASOL. Carbon dioxide provides a further example, with column measurements from the SCIAMACHY instrument on Envisat followed by those from the dedicated GOSAT and OCO-2 missions, with continuation provided by at least OCO-3 and GOSAT-2, supplemented by upper tropospheric measurements from high spectral resolution infrared sounders beginning with AIRS on EOS/Aqua and continued by instruments such as IASI and CrIS on operational meteorological platforms. An organizational framework for space agencies to coordinate their related activities for several individual variables or classes of variable is provided by the CEOS virtual constellations.

Several types of challenge have to be faced in seeking to ensure appropriate levels of continuity and innovation for key measurements, given the overall constraints on funding. Although the transfer of some types of observation from a research to an operational basis is generally to be welcomed, there remains a need for intermittent investigative missions for demanding variables. No simple rule exists as to when such missions might be justified, or when transition to routine operation should occur, as this will depend on the extent to which data from earlier investigative missions have been exploited to improve models or data analyses, and on the availability and costs of new technologies that could be deployed to measure the variables concerned. A particular case in point is that of active vertical profiling measurements of cloud and aerosol properties, for which arrangements beyond EarthCARE are uncertain.

The existence of a substantial gap in the provision of a certain type of observation is a particular issue when the use of such data is of demonstrated value for monitoring or forecasting, either as input or as routinely used diagnostic data. A prime example of this is the forthcoming gap in the limb-sounding of upper atmospheric composition and temperature that has been identified for several years as needing to be filled or minimized. This is discussed further in section 3.6.1 below. Gaps are more justifiable if they are related to new types of observation for which time may be needed to establish the value of the data provided or the robustness of the measurement technology. Examples are the measurements of ocean-surface salinity noted above and the wind 
measurements expected from ADM-Aeolus. In such cases mission planning needs to be agile so as to minimize gaps for types of observation that have been demonstrated to yield cost-effective benefits.

More generally, CEOS maintains an on-line "Mission, Instruments and Measurements" data base (MIMD; database.eohandbook.com), which provides information gathered from its members on their current and future space-based systems, with the future missions categorized as approved, planned, or considered. Other useful sources of such information include the WMO OSCAR database (www.wmo-sat.info/oscar/satellites) and the Earth Observation Portal provided by ESA (eoportal.org/web/eoportal/satellite-missions). Consulting such databases provides a good overall picture, although (as found when preparing this report) cross-checking and internet searches are needed on matters of detail relating to particular instruments, as these are prone to changes or errors that take time to be registered or corrected in the databases. The databases reveal that the prime meteorological variables and some others are indeed well covered, while others are less well catered for, to various degrees.

There are issues of continuity to be addressed even for the operational meteorological and Sentinel satellite systems discussed in sections 3.3 and 3.4. These include recognized needs to pay more attention now to factors important for climate (such as calibration, instrument and platform characterization, orbital control and stability) than for previous generations of weather satellites, and needs to address questions related to new launches or mission-lifetime extensions in the light of the varying degrees of health of the multiple instruments that are carried by many of these satellites. Change inevitably occurs from one generation of satellite-borne instrument to the next, but balances have to be struck between reproducing the capabilities of a preceding generation of instrument, so as most closely to preserve long climate records, and improving the capabilities of the new generation of instrument, so as to improve forecasting capability for example. Users have to adapt to these changes, in addition to dealing with the differences in instrumentation from one operator to another. This has become a demanding task, even for well-resourced centres, as the number of operators and instruments flown by each of them have both been increasing. The situation is ameliorated by collaborative institutional arrangements providing inter-calibrated, multiinstrument data records and products, common software and exchange of information on data quality and use.

Alongside issues related to the continuity of the space-based observations are issues concerning continuity of complementary in situ observations. Partly realized threats to reduce ground-station and shipboard measurements of atmospheric composition are one such. Observations from landbased meteorological networks have increased in number, but coverage is still far from uniform, and even generally welcome improvements such as increases since around the year 2000 in the number of available radiosonde observations may cause problems in integration with space-based observations unless care is taken to reconcile the biases of the different types of measurement (Simmons et al., 2014). Assessments of the changes over time in analyses of ocean temperatures and the accuracy with which they can be forecasted by seasonal prediction systems has to be carried out with care because of substantial observing system improvements such as the build-up of the TAO/TRITON array and introduction of altimetry in the 1990s, and deployment of Argo floats in the 2000s (Stockdale et al., 2011; Xue et al., 2012). 


\subsection{Observation of specific components of the Earth system}

Any one observation may serve many purposes: verifying the forecasts made days, months or seasons previously, initialising the forecast for days, months and seasons ahead, supporting the development or quality assurance of improved models over future years, calibrating the forecasts produced by these models, and characterising climate through repeated use over decades or more ahead as methods of reprocessing and reanalysis are improved. Moreover, any one observed variable may be of importance for more than one physical domain, for more than one of the physical and biogeochemical cycles of the Earth system, or for more than one area of societal benefit. In turn, any one measurement from space may contribute to the provision of information on more than one variable.

Accordingly, there are multiple ways of presenting the status and requirements for specific elements of Earth observation, each with their own advantages, disadvantages and applications. The discussion in this section is split by the atmospheric, oceanic and terrestrial domains, but with separate discussions of observations and other data for the cryosphere and for the carbon cycle. It touches on many important topics, but is not intended to be exhaustive. A more comprehensive variable-by-variable account, including many illustrations of data coverage, is provided for the ECVs by GCOS (2015).

\subsubsection{Atmosphere}

\subsubsection{Temperature and wind}

Temperature and wind are fundamental meteorological variables that affect many aspects of the working of the Earth system. Their observation is essential to making weather forecasts and monitoring the climate system, and as such they are relatively well provided for by operational in situ networks and the satellite observing systems discussed in section 3.3. Wind observation from space is more challenging than temperature observation, but data assimilation systems infer information on the wind from the more plentiful observations of temperature through the balance constraints that are effective in the extratropics, and wind information may also be extracted from observations of variables that act as tracers in some parts of the atmosphere.

Aside from continuation and refinement of the valuable provision of information on temperature (and other variables) from passive microwave and infrared sounding, required enhancements of routine space-based observation include establishing a full operational constellation of receivers for inferring vertical profiles of temperature (and humidity) from the occultation of GNSS signals, where current arrangements and short-term plans are for receivers on the operational Metop, Metop-SG and FY-3 platforms, with further, plentiful coverage from the Taiwanese/US constellations of COSMIC and COSMIC-2 receivers and additional coverage from receivers on individual satellites such as GRACE and its planned follow-on. There are opportunities to improve coverage by using GNSS constellations other than the GPS used hitherto (Liao et al., 2015). Establishment of fully operational arrangements are important not only because of the direct value of this type of observation for weather forecasting and climate monitoring, but also because the stability of these measurements and of measurements from operational high spectral resolution infrared sounders, allied with reference measurements from the GRUAN, provides a baseline for calibration of data from other components of the observing system (WMO, 2014). Valuable temperature information in the upper stratosphere and mesosphere is also provided by the limb-emission measurements that provide 
much information on atmospheric composition. The future of this type of measurement is a concern that is identified in several place in this report.

A further required enhancement is for flight of scatterometers in the afternoon sun-synchronous polar orbit to complement the data on surface winds over sea currently provided or planned from instruments flown in morning orbits. Different and welcome in the roadmap for coming years is the ADM-Aeolus mission, aimed at demonstrating the value of applying challenging Doppler lidar technology to determine wind profiles from space.

Gaps in spatial coverage by in situ measurement are a longstanding concern, especially close to the surface, and the future balance between radiosonde, aircraft and space-based upper-air observation merits reconsideration in the light of recent and imminent developments. The relatively long history of observations of temperature, wind and related meteorological variables, notably surface pressure, brings with it particular requirements and opportunities for data recovery, reprocessing and reanalysis to improve monitoring and understanding of climate variability and trends. This includes reprocessing satellite data to benefit from improvements over time in extracting wind information from successive images, as undertaken originally using data from geostationary orbit but now established also for polar regions, with emerging products for other regions derived from pairs of satellites in similar polar orbits (Borde et al., 2016).

\subsubsection{Water vapour and cloud}

Measurements of water vapour and cloud are important because of the role these variables play in radiative transfer, the formation of precipitation and latent heat release, and thereby in the forcing of atmospheric motion and the feedback effects that amplify or otherwise interact with climate-change signals. They also play an important role in atmospheric chemistry.

Sounding from space in both the infrared and microwave is the primary source of observational data on middle and upper tropospheric humidity. Microwave imaging provides data on the net water-vapour content of the lower troposphere over sea. In situ measurement remains the primary source of information for the boundary layer and lower troposphere over land, although information on the integrated water-vapour content comes from the delays in receipt of GNSS signals by groundbased receivers. Future provision of operational observation from space is closely tied with that for temperature, and largely subject to only the same minor concerns. Detail can be found for example by examining WMO's OSCAR database (www.wmo-sat.info/oscar/observingmissions). Routine in situ humidity measurement from commercial aircraft, supplementing current temperature and wind measurements, is beginning to be established.

Near-tropopause and lower stratospheric water vapour is difficult to measure because of its very low values, yet it matters in terms of its direct radiative effects and source of moisture for cirrus clouds. Here there is a need for expanded high-quality in situ measurement, such as envisaged to be provided by the GRUAN, as well as aircraft observation and limb-sounding from space. Lack of future provision for the latter is a major concern, as discussed below for other trace constituents.

Biases in both observations and models have been particularly prevalent for water vapour over the years, from the boundary-layer upwards. This has caused issues in particular for data assimilation, as evident in problematic humidity and precipitation products from reanalysis. Links between near-surface tropical temperature change and temperature and humidity change in the 
tropical upper troposphere have been difficult to reconcile between observation and modelling, but several recent studies (Lott et al. 2013; Mitchell et al., 2013; Simmons et al., 2014) point to a much improved situation.

Data are needed on the horizontal and vertical distributions of clouds, and on cloud microphysical properties. Satellites have long provided information on the horizontal distributions of cloud, with the work of the International Satellite Cloud Climatology Project (ISCCP) over more than thirty years deserving particular mention. The vertical distributions of clouds and in-cloud droplet distributions needed to improve process parameterizations in models are now becoming available, drawing on data from CloudSat, CALIPSO and CERES, and should be improved with data from EarthCARE. In addition, assimilation of cloud- and rain-affected radiance data from operational sounders and imagers has become a focus of research and development for numerical weather prediction, and WCRP has established a grand-challenge research focus on clouds, circulation and climate sensitivity (Bony et al., 2015). There are thus reasons to be optimistic that significant progress will be achieved over the coming ten years.

\subsubsection{Precipitation}

Precipitation is a primary input for the surface hydrological cycle. Measurements of characteristics such as the amount, frequency, intensity and type of precipitation are crucial for understanding and predicting the Earth's climate, weather, streamflow, soil moisture and water availability. Gauges generally give quite accurate point measurements of rainfall, although prone to undercatch. The spatial and temporal variability of precipitation leads to considerable uncertainty in precipitation maps for areas without data from dense gauge networks, especially where terrain is complex, and data from such networks are often not made freely or promptly available. Highfrequency observations are needed to assess rates of precipitation. Surface-based radar observations offer denser spatial coverage and provide instantaneous rates, but accumulated amounts are less certain than those from most gauge arrays. Integrated measurements, derived from combinations of space- and ground-based data, give more reliable areal estimates where dense coverage is lacking on the ground.

Estimates of precipitation from space are made predominantly from passive remote sensing in the spectral range from the visible to the microwave. The precipitation radar on the TRMM satellite provided an invaluable record of tropical precipitation following launch in 1997 until its operation ceased in April 2015. The Indian-French Megha-Tropiques, launched in 2011, provided more frequent observations within the tropics from a lower inclination orbit using microwave radiometers. The NASA/JAXA Core Observatory satellite of the Global Precipitation Measurement (GPM) mission is currently making radar and microwave measurements with much broader orbital coverage to $65^{\circ}$ latitude and greater sensitivity to light rain and snowfall, providing a basis for unifying the microwave measurements of precipitation from a constellation of satellites operated by several partners (Skofronick-Jackson et al., 2013).

The most comprehensive precipitation estimates come from hybrid products that combine in situ and space-based measurements. Perhaps the most widely used is that provided by GPCP, the Global Precipitation Climatology Project. Over land, GPCP makes extensive use of the gauge-based analyses of the GPCC, the Global Precipitation Climatology Centre (Becker et al., 2013). Known shortcomings of the GPCP estimates over sea, which utilizes both passive microwave and infrared measurements, 
have recently been corrected using data from the Cloudsat cloud profiling radar combined with data from either the TRMM precipitation radar or AMSR-E (Behrangi et al., 2014).

Notwithstanding the improvements brought by GPM, a significant shortcoming remains at high latitudes, particularly over the gauge-free regions of the Arctic and Southern Oceans. The suite of satellite sensors presently available are largely unable to differentiate between cold or icy surfaces and frozen precipitation. Although useful precipitation climatologies can be assembled from missions like CloudSat and the future EarthCARE, such missions do not carry sensors designed for the purpose. Future sensing capability complementary to what is provided by missions such as GPM is needed to constrain knowledge of solid and liquid precipitation in polar regions.

\subsubsection{Aerosols}

Aerosols influence the heat balance of the atmosphere directly by scattering and absorbing radiation, and indirectly through influencing cloud reflectivity, cover and lifetime. Tropospheric aerosols include dust, sea salt, secondary organic aerosols, primary biomass burning aerosols including black carbon as well as secondary anthropogenic organic and inorganic aerosols related to industry, transport and agriculture. Aerosols near the surface of the Earth damage health and disrupt transportation. Long-range movement of dust redistributes mineral nutrients. In addition, the aerosol distribution also needs to be taken into account in retrieving information on other variables from space-based measurements. This includes trace-gas concentrations and some land and ocean properties, ocean colour for example.

Understanding and monitoring the role of stratospheric aerosol in climate is important not only because of the significant warming of the stratosphere and cooling of the troposphere by sulphate aerosol that results from major volcanic eruptions, but also because artificial enhancement of stratospheric aerosol has been proposed as one of the geoengineering approaches to offsetting tropospheric warming due to increased greenhouse gases.

Data on aerosols will continue to be provided by a number of the long-term satellite missions, including from the satellites in the afternoon orbit of the JPS, from Sentinels 3, 4, 5p and 5, from GCOM-C and from $3 \mathrm{MI}$ on Metop-SG, and by research missions such as EarthCARE. Occultation and limb scattering data, for which continuity of long-term provision is less certain, have also proved important for estimating stratospheric aerosol of volcanic origin (Vernier et al., 2011; von Savigny et al., 2015). There are, in addition, important needs for data from in situ networks and airborne programmes.

Highly calibrated ground-based measurements are needed to support the retrieval of aerosol optical depths and other aerosol properties from space-based measurements. The AERONET network of sun photometers (aeronet.gsfc.nasa.gov) has provided the "gold standard" for determining values of aerosol optical depth, and its data have been used in particular for the validation and bias correction of MODIS and MISR retrievals and model-based products.

Lidar-based ground networks provide vertical distributions of aerosols, and need to be sustained. In situ measurements from dedicated aircraft campaigns are also needed, to define more highly resolved aerosol properties. These include understanding the composition and size distribution of aerosols at altitude as well as their ice nucleation and cloud condensation nuclei properties. 
Development of the IAGOS (In-Service Aircraft for a Global Observing System) programme offers the prospect of long-term measurement from instrumented commercial aircraft.

Surface-based networks are also needed for aerosol composition and size distribution. In the USA the IMPROVE network has proved invaluable. Many regional networks have been folded into the WMO-sponsored GAW network. Measurements of particulate matter from air-quality networks have also been utilized to verify outputs from global analysis and forecasting systems. Several international initiatives should help develop the better links required between environmental agencies and weather services to ensure progress in data exchange and service provision in general over the next decade and more. These include WMO's GAW Urban Research Meteorology and Environment project and Sand and Dust Storm Warning Advisory and Assessment System, a number of activities of the Copernicus Atmosphere Monitoring Service, AirNow International and the work of the community of practice for air quality under the Health SBA of the GEOSS. Many of these considerations apply also to ozone and other traces gases implicated in poor air quality.

\subsubsection{Ozone}

Ozone is a variable of particular importance because of the various significant roles it plays: in the stratosphere where it shields life below from the harmful effects of ultraviolet radiation and is linked through radiative forcing with temperature and circulation changes, in the troposphere as a greenhouse gas and at the surface as a gas affecting human health and a cause of reduced crop yield and forest growth. Controls on the anthropogenic substances that deplete ozone in the stratosphere, the strong global-warming potential of the substances that replace them, measures to improve surface air quality and changes in ozone concentrations due to climate change in general, confound the picture in the long term and add to the need for measurements and modelling.

Nadir-viewed measurement from space of ozone and other trace gases discussed in the following section is firmly established on the roadmap for the coming ten years, with a number of approved or planned missions identified in the CEOS database, including contributions from both operational meteorological systems and members of the Sentinel series. Novel observation from geostationary orbit will be provided not only by Sentinel-4 but also by the US TEMPO and Korean GEMS missions. Use of a highly elliptic Molniya orbit to focus on the Arctic is a Canadian mission that is under consideration.

A serious concern is the lack of future provision of the limb-sounded data that provide much better vertical resolution of the upper troposphere and stratosphere for ozone and several important species, including water vapour and ozone-depleting substances. Microwave limb sounding is currently provided from NASA's EOS-Aura satellite and the Odin satellite of a Swedish-led international mission. Both satellites are operating well beyond their nominal lifetime. This type of sounding is referred to next in the CEOS timeline only in the Global Atmospheric Chemistry Mission under consideration by NASA for launch in 2030, though as a proposal that survives from the US Decadal Survey published in 2007 it is likely to be reviewed in the successor survey currently taking place. Limb backscatter measurement of ozone is provided on NOAA's Suomi NPP satellite, and is scheduled for JPSS-2, but not JPSS-1, indicating that a gap in data provision is likely (Figure 4), although measurements expected to be made by FY-3E from 2017 and then FY-3G offer an alternative source of such data. Also, SAGE-III on the International Space Station should provide data based on solar occultation from 2016. 
A further concern is decline of the baseline in situ networks for total and profile ozone measurements provided respectively by Dobson and Brewer spectrophotometers and by ozonesondes.

\subsubsection{Other fast-reacting trace gases}

Gases such as nitrogen dioxide, sulphur dioxide, formaldehyde and glyoxal have to be measured because of their intrinsic importance for air quality and also because of their role as precursors for the formation of secondary aerosols and ozone in the troposphere. Other gases such as carbon monoxide are important because of the related chemical reactions in which they are involved. It has already been noted that methane also plays a role here, in addition to that of a greenhouse gas. Although measurement from space is more challenging for some of these gases than for ozone, the situation in summary is much the same for them as for ozone, with the absence of limb sounding the major concern.

As in the case for aerosols, the need remains for ground-based remote sensing and in situ measurement of near-surface and tropospheric profiles or column values for the fast-reacting trace gases, including measurements from the IAGOS programme. For many reactive gases a groundbased system is either not in place or provides only sparse coverage. Moreover, threats to the continuity of some long-term measurements from ground stations are becoming increasingly apparent. This in turn poses a serious threat to the observing system as a whole, as the groundbased data provide a crucial reference for effective product generation and integrated use of data from multiple satellite missions.

\subsubsection{Radiation budget}

The primary observations related to the Earth's radiation budget are of solar irradiance, the external driver of the Earth system, and of the reflected solar and emitted longwave radiation that leaves the atmosphere. The observations are made from space, and continuity and stability of measurement are essential for detecting fluctuations and change. The inferred imbalance between incoming and outgoing fluxes is smaller than the uncertainty of several $\mathrm{Wm}^{-2}$ in the measurements of outgoing radiation, although as uncertainty stems mostly from biases, changes over time can be tracked. Measuring the variability of fluxes in space and time over the globe thus provides insight into the overall behaviour of the climate system, and provides vital data for the evaluation and improvement of climate models.

Broadband measurements of outgoing radiation have been made since the 1970s. In particular, the CERES instrument on NASA's Terra satellite has provided data for more than fifteen years, with instruments also now flying on the Aqua and Suomi-NPP platforms, and a final one scheduled for flight on JPSS-1. Measurements will be continued with a new instrument on JPSS- 2 and from instruments on FY-3 satellites. The Geostationary Earth Radiation Budget instrument on the current Meteosat series of geostationary satellites provide complementary broadband measurements with good temporal sampling of the diurnal cycle but partial spatial coverage.

Total solar irradiance has also been measured since the 1970s. There is considerable variation in the absolute values that have been given by different instruments; lower values are provided consistently by the latest, and best-calibrated, instruments to fly, including from the long-lived SORCE mission. 
Radiation at the Earth's surface is a fundamental component of the surface energy budget that is crucial to many aspects of the working of the Earth system, including its energy and hydrological cycles. Systematic ground-based observations are needed for monitoring climate variability and change, and for evaluating products based on satellite data and from reanalyses and model runs. The Baseline Surface Radiation Network is of particular importance in this regard. Data are also needed for the siting and operation of solar power-generation systems, and for agriculture, health protection and tourism.

Generation of data products from the space-based observations makes use of radiative transfer modelling and ancillary data on several of the atmospheric and surface variables discussed in other sub-sections, bringing further requirements for their observation and analysis. This is especially the case for the derivation of fluxes at the Earth's surface.

\subsubsection{Ocean}

Improved understanding and quantification of the state of the ocean is needed at multiple spatial and temporal scales, in particular with regard to:

- mesoscale and sub-mesoscale processes and dynamics and their interactive role and impact on energy transport, marine ecosystems and biogeochemical cycles;

- physical and biogeochemical air/sea interaction processes and the subsequent transfer of heat, gases and nutrients within the oceans by turbulence, mixing, convective motion and biological pumping;

- regional and global sea level;

- forecasting oceanic and interacting Earth-system conditions over all time scales.

Oceanic circulations are largely driven by fluxes of heat, momentum and fresh water at the ocean surface. Modelling and data assimilation for the ocean thus have a predominant need for goodquality atmospheric data and data on the input of fresh water from rivers. Linked to these inputs is the need for data on ocean-surface variables such as SST, sea-surface salinity (SSS), surface current and sea state (ocean-surface-wave conditions). This has to be complemented by data on the subsurface state of the ocean from both in situ and remote-sensed observations, in particular to constrain models used in estimating the ocean state.

A number of aspects of observation of the ocean are discussed below in this section, 3.6.2, and also in subsequent sections on the cryosphere and biogeochemistry. Important ocean observations not covered further here include those of ocean transports across sections, often derived (such as RAPID or WOCE), but also directly measured by cable (Florida Strait transport). Mention should also be made of the potential of a synergetic integrated approach to consistently fuse available and planned satellite data (high-resolution SST, sun glitter reflectance, ocean colour and radar images, and lower resolution sea surface topography and scatterometry observations) with in situ measurements and fine-resolution numerical-process models to advance understanding of behaviour of the upper ocean on horizontal scales of 20 to $100 \mathrm{~km}$. 


\subsubsection{Sea-surface temperature}

In addition to their importance for ocean-state estimation, datasets on SST are combined with analyses of surface air temperature over land to form the global surface temperature records that provide basic metrics used to quantify global warming. They are important also for numerical weather prediction, marine services and atmospheric reanalysis.

Infrared instruments on polar satellites provide information with global coverage and good horizontal resolution and accuracy, except in areas that are persistently cloud-covered, where data from passive microwave instruments have been shown to be complementary. Observing the diurnal cycle is becoming increasingly important, for which geostationary satellites offer capabilities. Arrangements for future space-based observations as summarized in section 3.3 are generally regarded as adequate, but with some concern over the extent of the provision of passive microwave measurements. Ships and buoys provide SST observations of good temporal frequency and accuracy. Coverage has been marginal or absent over some areas of the Earth, but recent improvements in the in situ network have enhanced coverage considerably.

\subsubsection{Sea-surface salinity}

The salinity as well as temperature of the ocean affects density and therefore ocean currents. There is large uncertainty in the net flux of fresh water into the ocean from precipitation, evaporation and river discharge, which affects SSS and mixed-layer properties. There is also large uncertainty in the fresh-water flux between sea-ice and sea-water. The fresh-water flux is probably the largest source of uncertainty in the estimation of ocean salinity in the upper $100 \mathrm{~m}$. Salt exchange between ocean and sea-ice is believed to influence the large scale thermohaline circulation, thereby affecting the deep ocean. SSS also plays a significant role in El Niño events. It is quite widely measured from moored buoys, but these data are not sufficient to resolve the variability of the equatorial salinity front, whose horizontal scale is $100-200 \mathrm{~km}$. Near-surface salinity measurements from Argo floats (see 3.6.2.5) are also important. Data of the type provided by the Aquarius/SAC-D and SMOS satellites are potentially useful, but provide limited information for cold sea temperatures and there is little experience to date indicating their general value in data assimilation for seasonal forecasting or reanalysis. They may support the detection of the SSS variability associated with EI Niño events and migration of fronts in SSS, and their use with SST data to constrain estimates of the flux of $\mathrm{CO}_{2}$ between atmosphere and ocean is being explored.

\subsubsection{Surface Current}

Satellite altimetry is arguably the most mature technique for mapping ocean surface currents and has permitted breakthroughs in understanding the dynamics of the ocean circulation on scales above about $100 \mathrm{~km}$ and eddy kinetic energy on a global scale. Altimetric data are regularly assimilated in ocean models and are highly important for marine service provision. Space-based estimates of ocean surface current and higher-level derived quantities such as frontal boundaries can also be derived from SAR data and from passive measurements in the spectral range from the visible to the microwave.

In situ current measurements from moored buoys, surface drifters, coastal HF-radar installations, Argo floats, gliders and ship observations complement these satellite measurements. Each of the satellite and in situ measurement techniques has specific strengths and limitations, related to resolution, coverage, accuracy, depth-integration, cloud-dependence, empirically based retrieval 
methods and so on. By development and use of systematic data merging and sensor synergy, combined with advanced processing tools and simulation models, the complementary use of these sensing techniques can be optimized. Deficiencies are thereby reduced and the final surface current estimate is more consistent, regular and reliable.

\subsubsection{Sea state}

Coupling of atmospheric and surface-wave models is well established for combined weather and sea-state forecasting, having been used since 1998 at ECMWF (Janssen, 2004). It has also recently received attention in the context of the modelling of climate change (Fan et al., 2013, 2014). Evidence is growing of the importance of surface wave dynamics for the evolution of currents and temperatures in the upper ocean. Effects include those of wave breaking, generation of turbulence by Langmuir circulation and refraction of waves by currents. Interactions are two-way, and Earthsystem models with tightly coupled atmosphere, ocean-wave and ocean-circulation components should emerge over the coming decade.

Space-based data on sea state for assimilation and model evaluation have been provided by the ERS and Envisat radar altimeters as noted earlier, succeeded now by the Jason-2, CryoSat and Altika altimeters. SAR data from ERS-2 and Envisat have also been used. Satellites in the Sentinel series (Figure 5) will ensure long-term provision of these types of data. In situ data from moored buoys are important for independent evaluation of analyses and forecasts.

\subsubsection{Sub-surface temperature, salinity and other variables}

Sub-surface in situ data either on temperature alone or on both temperature and salinity are provided primarily by XBTs (Expendable bathythermographs), CTD (Conductivity, Temperature and Depth) transects, moored buoys and Argo floats. More recently, data under ice have been provided by instrumented seals and tethered profilers. Efficient autonomous underwater vehicles, so-called gliders, also now provide data on temperature, salinity and currents. Arrangements are in place for supply of various types of data both in near-real-time and from long-term repositories. Version control of datasets is a requirement as the applied bias corrections and quality-control decisions evolve over time and are important for estimating long-term changes. Examples are XBT depths (Wijffels et al., 2008) and data from the Argo pressure and salinity sensors (Willis et al., 2007).

Figure 6 shows time series and spatial distribution of the different in situ observations used in ECMWF's recent ORAS4 ocean reanalysis (Balmaseda et al., 2013a). The spatial and temporal sampling varies substantially between instruments: the XBTs usually follow commercial ship routes, CTDs are associated with intensive scientific field campaigns and the moored arrays sample the equatorial oceans at selected positions. Argo is the only observing system that samples the subsurface of the ocean fairly uniformly, measuring temperature and salinity down to depth of $2000 \mathrm{~m}$. Notwithstanding the marked improvements in the global in situ data coverage Argo provides, the deeper ocean and ice-covered regions remain largely unobserved. The deep ocean is important for decadal prediction and climate change. It is also important for shorter time-scale data assimilation, especially for maintaining the stability of the water column when assimilating partialdepth temperature and salinity profiles, and for imposing observational constraints on the multivariate relationships used in the assimilation of altimeter data, which can otherwise very easily contaminate the analysed deep-ocean variability. 



Argo: 0 Moorings: 30 CTD: 4605 XBT: 6341 Seals: 0

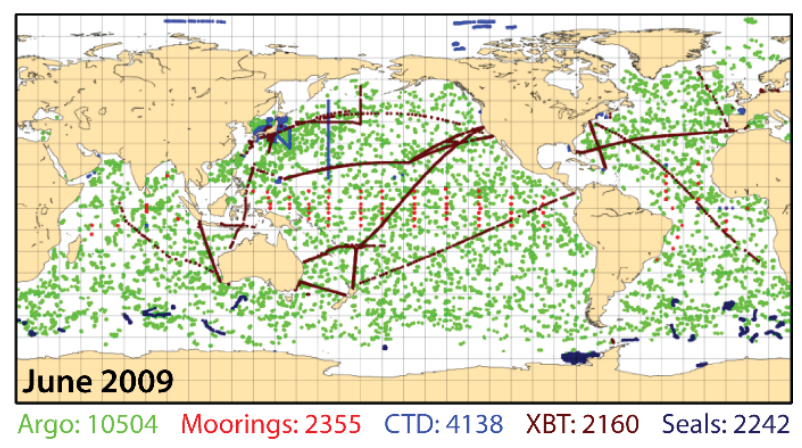

Figure 6 (left) Number of temperature (top) and salinity (bottom) observations within the depth range $400 m-600 m$ as a function of time per instrument type. The black curve is the total number of observations. The orange curve shows the number of assimilated observations. (Right) Observation coverage in June 1980 (top) and in June 2009 (bottom). Note that the colour coding for the instruments is not the same in the left and right panels. Data are as used in ORAS4. Source: ECMWF.

It is unlikely that any in situ observing system could provide sufficient global sampling of the ocean mesoscale. However, the in situ observing system for subsurface temperature and salinity should guarantee sustained broad-scale sampling of the whole ocean, including depths below $2000 \mathrm{~m}$ and ice-covered seas. The frequency, horizontal and vertical resolution of sampling should vary with depth and with location: more intense sampling is needed in boundary regions and along the equator, and close to the surface, while coarser and less frequent sampling may suffice for the deep ocean. Guidance is provided in the recommendations of a recent workshop on the Tropical Pacific Observing System (GCOS/GOOS/WCRP, 2014). The ongoing development of new Argo floats that descend much deeper than those presently deployed routinely offers promise for deep-ocean observation, and it is important that repeat hydrography be undertaken as envisaged under the international Global Ocean Ship-based Hydrographic Investigations Program (GO-SHIP; www.goship.org/). A wider strategy for deep-ocean observing of the range of key variables, including biological and chemical ones, is under development (Heimbach et al., 2014) under the auspices of the GOOS, and should guide progress over the coming decade. A strategy for observation under Antarctic sea ice is reported by Rintoul et al. (2014).

\subsubsection{Sea level}

Sea level integrates changes of several components of the climate system in response to anthropogenic and natural forcing factors and is an important indicator of climate change. A series of altimeters has delivered sea-level information continuously since late 1992 (Ablain et al., 2015). Data are currently provided by Jason-2, CryoSat and Altika; future provision has been referred to in section 3.3. The relatively high-resolution homogenous global sampling provided by these satellite data complements what is provided by the Global Sea-level Observing System (GLOSS; IOC/GOOS/JCOMM, 2012) core in situ network of about 300 tide gauges reporting in near-real time, 
which has other important applications such as tsunami warning and storm-surge monitoring and modelling. Also needed are geodetic measurements, as account must be taken of land movement, in particular due to glacial isostatic adjustment and human influences such as subsidence in coastal regions due to urbanization, water and mineral extraction and river management. This is needed in general to determine vulnerability of populations to inundation and to develop adaptive responses, and specifically at tide-gauge locations so that data can be used for study of long-term change. Here satellite systems play a role through use of GNSS signals and laser ranging from ground stations, and through data from orbit such as provided by the DORIS instruments carried on several satellites.

Along-track altimeter-derived sea-level anomalies (SLAs) are widely used in ocean data assimilation. Ground processing is consistently carried out to remove tides, apply the inverse barometer correction, make orbital corrections and cross-calibrate data from different instruments. Challenges are discussed by Fu and Haines (2013). The mean dynamic topography that is needed to utilize the SLA data may be taken either from a free run of the assimilating model, from a reanalysis or from direct use of other observational data, including from satellite gravity missions.

Global sea-level variations from altimetry may also be used to constrain the global fresh-water and ocean heat-content budgets.

Gravity missions such as GRACE (Tapley et al., 2004) and GOCE (Rummel et al., 2011) have provided original and highly important new information on the geoid. This opens, for example, the possibility of using of altimeter-derived sea-surface height as opposed to SLAs in ocean data assimilation systems, bypassing issues in determining the mean dynamic topography. Gravity missions also provide information on bottom pressure, which can be used globally to constrain the non-steric part of sea-level variations. Gravity-derived variations of the global mass field are also useful for verification of ocean reanalyses.

\subsubsection{Ocean colour}

Information on the concentration of the pigment chlorophyll-a in phytoplankton, and thereby on the biomass of phytoplankton, is provided by measurements of ocean colour. These are of vital importance because phytoplankton lie at the base of the ocean food chain and play a role in the carbon cycle through the photosynthetic production of organic matter from dissolved inorganic carbon and nutrients. Ocean-colour data also provide information on dissolved organic matter and on suspended particles in coastal waters. Space-based passive instruments sense only an upper layer of the ocean (ranging from 40-60m deep in the open ocean to less than $1 \mathrm{~m}$ deep in turbid coastal waters), and need to be coupled with in situ sampling of ocean colour and ecosystem variables, and models, to provide a complete picture.

Established data records on ocean colour from the SeaWiFS, MERIS and Aqua-MODIS instruments are being combined into a record from 1997 onwards under the ESA CCI and the Copernicus Marine Environment Monitoring Service, with similar activity under NASA's MeASUREs project. Continuity of measurement is being provided by OCM on Oceansat-2 and VIIRS, with future provision also from $\mathrm{OLCl}$ on Sentinel 3 and SGLI on GCOM-C. Novelty is provided by Korea's GOCI instrument, which provides data for a limited region around the Korean peninsula from geostationary orbit, raising the possibility of a future constellation monitoring key coastal waters. 


\subsubsection{Bathymetry}

Data on the bathymetry of the oceans has come primarily from shipboard sounding, supplemented by estimates derived from ocean surface elevation measurements using space-based radar altimetry. New measurements from the altimeters on CryoSat and Jason-1 have provided the source for a recent more-accurate bathymetry (Sandwell et al., 2014). There is also a potential for the time-varying bathymetry of shallow coastal seas to be derived by combining optical and SAR data (Pleskachevsky et al., 2011).

\subsubsection{Land}

A wide variety of data is needed concerning the land. Discussion in this section covers the required observations related to the basic characteristics of its surface and sub-surface, its vegetation, the water it holds and releases, and the related occurrence of fire.

\subsubsection{Surface and sub-surface characteristics}

Earth-system models require data on the physical characteristics of the surface and sub-surface of the land. Elevation of the surface is needed to specify both the model's resolved surface height and fields related to parameterizations, such as surface roughness and slope-related fields used to estimate generation of unresolved gravity waves, partition rainfall into interception, infiltration and run-off, and so on. This requires access to datasets with resolutions of order tens of metres, much higher than those of the models themselves. Use may be made of space-based digital elevation models (DEMs) based on either radar or optical measurements, such as from the SRTM and ASTER instruments respectively. Requirements for resolution and accuracy will become more stringent as model resolution increases and parameterizations are refined; needs over the coming ten years may be met by new datasets such as from the TerraSAR-X and TanDEM-X satellites.

Data on the type of land cover is a further requirement, including historic data on changes in land use. Models utilize information down to sub-grid-scale resolution on the presence of bare soils, vegetation of various types, water, snow and ice, and urban or other built-up areas. Data on soil morphology are also required, particularly as it affects drainage and retention of water. Current and near-future requirements for data from space appear to be catered for, in quantity at least, in view of the number of land-cover missions identified in the CEOS database.

Surface albedo is both a forcing variable affecting the climate and a sensitive indicator of surface environmental change. In Earth-system models it is typically in part prescribed from observations, and in part modelled in terms of variables that change dynamically, especially snow and sea-ice cover. Space-based data come from multi-spectral imager radiances from instruments such as MODIS and MERIS. ECMWF, for example, uses monthly mean fields of snow-free direct and diffuse albedo in UV-visible and near-infrared bands built from 16-day MODIS data accumulated over the years 2000-2003 (Schaaf et al., 2002). Multi-angular viewing, by instruments such as MISR and POLDER, adds the capability for providing near-instantaneous values. Generation of a multi-decadal record from a constellation of geostationary satellites is reported by Lattanzio et al. (2013).

\subsubsection{Vegetation}

Observations of the type and condition of vegetation are of direct importance for monitoring agriculture and the extent and health of forests and other biomes. They are also needed for use in Earth-system models, either to prescribe the characteristics of vegetation in models used for short- 
term prediction and some climate applications, or for initialization and validation of climate models that include a representation of the dynamic evolution of vegetation. Both are areas that are expected to develop in the next ten years, as forecasting and reanalysis models move from climatological prescriptions to use inter-annually varying values determined by data assimilation, and as climate models continue to be refined. The importance of the observation of vegetation has resulted in the establishment of two substantial GEO initiatives in recent years: for Global Agriculture Monitoring (www.earthobservations.org/geoglam.php) and the Global Forests Observation Initiative (www.gfoi.org).

Much of the space-based data that are required comes from instruments that have already been mentioned in other contexts. High spatial resolution measurements in the visible and near infrared such as from the Landsat and Sentinel- 2 imagers provide detailed maps for monitoring changes in coverage and type of vegetation, for use in high-resolution modelling and for comparison with in situ data. Moderate-resolution imagers such as MODIS, MISR and MERIS, and successor instruments such as flown on both operational meteorological and Sentinel platforms, provide data for deriving products such as Leaf Area Index (LAI) and Fraction of Absorbed Photosynthetically Active Radiation (FAPAR). LAl data are used in the specification of vegetation in models (e.g. Boussetta et al., 2013a) and together with FAPAR (Gobron and Verstraete, 2009) to estimate the gross primary production of biomass by photosynthesis, an important component of the carbon cycle. A new capability for providing more-direct data for the latter from remote sensing of solar-induced chlorophyll fluorescence has been demonstrated using data from the GOSAT greenhouse-gas mission (Frankenberg et al., 2011; Joiner et al., 2011), and is expected to be enhanced by the availability of data from OCO-2 and GOSAT-2. Measurement of above-ground forest biomass is the objective of the 7th ESA Earth Explorer mission Biomass, whilst observation of field-scale vegetation fluorescence emission is the objective of the 8th Earth Explorer mission FLEX. These missions are scheduled for launch around 2021 and 2022, respectively.

Knowledge of the structures of vegetation canopies is also needed for better process parameterizations of plant functional types. Ground-based survey data and space-based multiangular instruments or radar data have started providing such information, but this is still in research mode.

Other in situ measurements are discussed in the context of the carbon cycle in section 3.6.5.

\subsubsection{Land temperatures}

Earth-system models typically include the temperature of several soil layers and one or more overlying layers of snow. Observational data are required for evaluating these models, even if acquisition of direct data to initialize predictions has not been a priority, notwithstanding the importance of soil temperature to agriculture. Data on the sub-surface temperature of the land is of particular importance where freezing occurs, especially in high-latitude and high-altitude regions where changes in permafrost may affect the stability and erosion of terrain, the release of greenhouse gases, sediment transport, the characteristics of vegetation and the amount of surface and sub-surface water.

Soil temperature is quite widely measured in situ for agricultural purposes but not exchanged internationally on a routine basis. Sites making borehole measurements of permafrost temperatures and sites monitoring the active layer above permafrost that is subject to thawing and refreezing as 
the year progresses form the Global Terrestrial Network for Permafrost; recent reports are provided by Noetzli et al. (2014) and Romanovsky et al. (2014).

Space-based data play a limited role in this case. Land surface temperature measurements from satellite are of a radiative skin temperature that is influenced by a number of variables, including vegetation and soil moisture, and are not readily related to soil temperatures, although the data have a range of related applications: to frost occurrence, urban heat, drought early warning and more. Concerning permafrost, satellite data can identify changes in lake area and numbers in regions where this may be due to changes in permafrost and seasonally-frozen ground regions, and passive microwave data can be used to map areas of frozen and thawed soil. SMMR and SSM/I data have been used to detect change in the near-surface soil freeze-thaw cycle (Kim et al., 2012), and the sequence of AMSR instruments provides an ongoing data record.

\subsubsection{Terrestrial water variables and fluxes}

Soil Moisture is an important variable for weather prediction and climate modelling. Both observational and forecast data are needed for agricultural and water-resource management, and many other applications. Indeed, a cross-SBA analysis by GEO (2010) ranked soil moisture second behind precipitation among the variables that were critical priorities for Earth observation from a direct user perspective.

Both active and passive space-based microwave measurements are used to estimate soil moisture. Mention has already been made of the dedicated SMOS (Kerr et al., 2012) and SMAP (Entekhabi et al., 2014) missions. Information on soil moisture is also provided by the series of AMSR and scatterometer instruments. ESA-supported projects have generated a first 30-year dataset of satellite soil moisture based on data from passive and active microwave sensors (Dorigo et al., 2012).

In situ soil-moisture measurements are needed for validating products derived from satellite data, for developing new integrated soil-moisture products, and for providing information at depths below those sensed in the microwave. Some of the national and regional soil-moisture networks set up for agricultural and other purposes participate in the International Soil Moisture Network (ISMN; Dorigo et al., 2011a, b) for validation of space-based estimates of soil moisture. There is, however, a general need for improved and more widespread operational hydrological ground networks and data exchange, especially for soil moisture. Current contributions to the ISMN are listed at ismn.geo.tuwien.ac.at/networks/Networks. Coverage can be seen to be especially poor over Africa and South America.

Evaporation and Evapotranspiration (ET) measurements can be used to estimate consumptive water loss, especially irrigation losses and non-productive evaporative losses. Accurate flux values at a particular location can be derived from in situ Bowen ratio and eddy covariance measurements over land, but estimating ET and closing the water balance for the continental and global scales remains challenging. ET is an important variable for water management. For agricultural areas, ET is the primary part of consumptive water loss and ET monitoring can be used by water managers to plan and assess water used in irrigation. ET is generally estimated from satellite data using a range of models, with inputs from the optical and thermal bands from the MODIS, MERIS, AATSR, Landsat, and other satellite sensors. Higher-resolution satellite thermal imagery is needed to provide better estimates for agricultural and other applications. 
In situ measurements of evapotranspiration (ET) include station-based conventional lysimetric and soil water balance methods, Bowen ratio energy balance, scintillometry and eddy-covariance. Standards or protocols for ET measurements, databases and metadata, including from FLUXNET and other tower networks, need to be better developed.

River discharge measurements have essential direct applications for water management and related services, including flood protection. They are also needed for evaluating the working of the hydrological cycle in Earth-system models and for use in the development and operation of floodmodelling components that are driven by or embedded within Earth-system models, or will be in coming years. While in situ methods are currently a cost-effective and in principle reliable option for streamflow measurements, the decline of some in situ networks, lack of data sharing or delays in making data available limit the capability to carry out global discharge monitoring and assessments. In view of the limited prospects for in situ networks, efforts are being directed at expanding the capability of satellite remote sensing to contribute to the estimation of river discharge. Candidates include imaging sensors that document water extent and lidar or radar altimeters that measure river heights. The imaging radar altimeter developed for the Surface Water and Ocean Topography (SWOT) mission will be able to combine the measurement of surface water extent with water height, and also measure the surface slope along the river channel.

Rivers are responsible for the transport of the majority of suspended sediments and their associated contaminants. River sediment transport strongly influences the quality and biodiversity of surface waters, riparian environments and the functioning of coastal zones. Sediment data need to be collected, archived and analysed so that linkages between river and lake processes and water quality can be fully understood. Rivers are also extensively used in industry especially for cooling and often result in changes in temperature of the waters as well as addition of pollutants. There is an increasing need to monitor the temperature as well as the quality of river waters.

Runoff and river discharge can be estimated using the Soil Conservation Service Curve Number method for determining the approximate direct runoff volume for a given rainfall event in a particular area. The advantage of the method is its simplicity and widespread inclusion in models (USDA-NRCS, National Engineering Handbook). SMOS data have recently been used in the method to define the potential maximum retention values for each grid point (Garcia-Leal et al., 2013; LopezBaeza et al., 2014).

Continental surface water storage pools (lakes, reservoirs, floodplains, wetlands and river channels) are home to aquatic ecosystems. Changes in the amount of water held in reservoirs have to be taken into consideration in accounting for sea-level changes (Church et al., 2013). Earth-system models require at least information on the location and areal extent of lakes and reservoirs, and may include explicit modelling of these water bodies, which brings a requirement for bathymetric data. Necessary information on surface temperature, ice cover and water level can be derived from various types of space-based measurement. Nevertheless, monitoring of standing water bodies in general remains poor. A diverse set of challenges has to be faced, but the need for data on terrestrial surface water storage requires that a strategy be developed for monitoring. This will have to combine in situ and satellite-based observation.

Groundwater is an important source of water in many areas. It is removed by natural processes (discharge) and pumping, and is replaced, in whole or part, by recharge, which is at a maximum 
during wet periods. Depletion of groundwater by pumping has exceeded impoundment of water in reservoirs in recent decades, giving a net anthropogenic contribution to sea-level rise from changes in terrestrial water storage, although estimates of magnitude vary quite substantially (Church et al., 2013). Natural variability also has to be taken into account. Inventories of groundwater resources are required, and changes must be monitored and forecast. In situ groundwater measurements are collected in many countries but few share these data. Groundwater is estimated routinely from lowresolution estimates of total water storage from space-based measurement of variations in gravity by the GRACE mission using a land-surface model. Continuation in the short term should be provided by the US/German GRACE Follow On mission planned for launch in 2017.

\subsubsection{Fire}

Fire is a variable that is important because it is a hazard, it emits greenhouse gases, other chemical species and aerosols that have to be taken into account in air-quality forecasts and climate projections, and it changes vegetation. Estimates of emissions for various purposes are based either on burnt-area, active-fire or fire-radiative-power products that are currently based primarily on data from the MODIS instruments. Operational continuity is expected to be provided by products from VIIRS and future imagers on polar meteorological platforms, and from the SLSTR instrument on Sentinel-3. Such data are supplemented by data from a number of the operational geostationary meteorological satellites, which provide better resolution of the diurnal cycle. Issues to be addressed in combining data arise from differences in viewing angle and spatial resolution. Other data required include those on vegetation and the peat content of soils. Inversion techniques (section 5.5) that use atmospheric observations of emitted species are likely to play an increasing role in the coming ten years. Kaiser et al. (2014) provide a recent discussion of issues, in the context of the fire data assimilation system established for the Copernicus Atmosphere Monitoring Service.

\subsubsection{Cryosphere}

\subsubsection{Ice sheets, ice caps and valley glaciers}

Most of the Earth's freshwater is stored in ice sheets, ice caps and valley glaciers. Ice mass is accumulated by precipitation, and ablated by melting, sublimation, and evaporation. The net balance between the inflow and outflow components of ice mass reacts sensitively to atmospheric warming and precipitation changes. Ocean warming is a further factor in the case of the accelerated movement and melting of the outlet glaciers that discharge directly into sea from the ice sheets of Greenland and Antarctica. In AR5, Stocker et al. (2013) note that the largest uncertainty in estimated mass loss from glaciers comes from the Antarctic, and that the observational record of ice-ocean interactions around both the Antarctic and the Greenland ice sheets remains poor. Overall, the currently prevailing ice-mass loss contributes about one half of the ongoing rise in global sea level. AR5 also reported substantial progress in ice-sheet modelling, particularly for Greenland, but expressed low confidence in the ability of current Antarctic ice-sheet models to capture the temporal response to changes in external forcing on the decadal to centennial time scale.

Requirements for in situ observations include continuation of process-oriented measurements needed for improved understanding and model development, maintenance of glacier-observing sites providing long-term mass-balance measurements and addition of sites in data-sparse regions, and undertaking of the measurements needed for calibration and validation of satellite data. Aircraft laser altimetry has a role to play in the latter. Space-based imagery, such as from Landsat, ASTER and 
Sentinel-2, is important for monitoring the areas of glaciers and ice caps, and DEMs as discussed in the section 3.6.3.1 are required decennially or thereabouts for calculation of volume changes. The main requirements for ice-sheet data from space are elevation changes from radar and laser altimetry, mass changes from gravimetric missions and movement from radar interferometry.

\subsubsection{Sea ice}

Data on the concentration or coverage of sea ice are required for basic monitoring of the physical climate system and for linking with monitoring of the impacts that changes in physical climate can have on vulnerable polar ecosystems. Data are also required for input to forecasting and reanalysis systems, which in the future can be expected increasingly to model sea-ice changes dynamically, and for verifying climate models that already include dynamic representations of sea ice. Comprehensive data on concentrations are primarily built up from passive microwave data, starting from SMMR in 1979. Some analysis is available of earlier data (Meier et al., 2013).

Determination of sea-ice thickness from space is less mature. Estimates based on ice freeboard are currently provided from high-inclination orbit by the CryoSat radar altimeter, with data from laser altimetry provided earlier by ICESat-1 and to come from ICESat-2. Use of data from the earlier ERA and Envisat radar altimeters is under development. Longer-term records can be constructed using data from optical sensors, starting with AVHRR and including MODIS and VIIRS, in conjunction with energy-budget modelling. Other space-based data on sea-ice include those on ice motion and other properties from SARs, on thin-ice thickness from SMOS and on melt ponds from MODIS.

Reliance on in situ data has to be made for sea-ice concentration in the pre-satellite era, where a need for data recovery and improved charting of sea-ice remains. Direct in situ measurements and airborne and submarine remote sensing have roles to play in the evaluation of space-based data on ice thickness. Measurements of snow depth and density are important also, as allowance has to be made for snow when deriving the thickness of sea-ice from measurements of freeboard.

\subsubsection{Snow}

Data on the coverage, depth, snow water equivalent and other properties of fallen snow are important for a number of reasons. Release by melting of water stored in the form of snow has implications for ecosystems, hydrology, naturally watered agriculture and a range of direct uses of water by humans. Snow cover, melt and runoff are also important elements in the working of the Earth system. Data on snow are accordingly important for initialization or evaluation on all time scales over which Earth-system models are applied: for weather forecasting (where the presence of lying snow must be well represented to avoid near-surface air temperature errors), sub-seasonal and seasonal prediction (where initial conditions on snow depth are important, and melting has impacts on soil moisture and the surface energy balance) and long-term climate simulations and projections (where snow/albedo feedbacks must be well represented and changes in snow climatology and the associated hydrology reliably identified).

Space-based observations are used to derive products on snow cover such as the NOAA multisensor product for the northern hemisphere, as used in some snow data assimilation systems for numerical weather prediction. The AMSR instrument provides data on snow-water equivalent, though with limited accuracy over difficult terrain and for deep-snow conditions. A critical requirement remains for in situ measurements of snow depth such as exchanged internationally from the WMO synoptic network and used also in near-real-time data assimilation. Here there is 
scope both for improved reporting standards and greater exchange of data from national networks. The need for datasets to support modelling and reanalysis brings additional requirements for recovery and exchange of historic in situ data. The development since 2011 of the WMO Global Cryosphere Watch provides a framework for improvement over the coming years.

\subsubsection{Carbon cycle}

Observation-based estimates of the spatial-temporal distribution of ocean-atmosphere $\mathrm{CO}_{2}$ fluxes rely mainly on measurements of the difference in partial pressure of $\mathrm{CO}_{2}$ between the atmosphere and the sea-surface $\left(\Delta \mathrm{pCO}_{2}\right.$; Wanninkhof et al., 2009, 2013). Measurements of carbon and related variables in the interior of oceans can also be used with models to constrain the uptake of anthropogenic $\mathrm{CO}_{2}$ in each ocean region. The fluxes in key regions, like the Southern Ocean, are poorly constrained as $\triangle \mathrm{pCO}_{2}$ measurements are very sparse, and uncertainties in gas exchange kinetics affect estimates of fluxes. Uptake of $\mathrm{CO}_{2}$ causes the ocean to acidify, which together with associated carbonate changes has consequences for marine organisms and ecosystems that AR5 notes are just beginning to be understood (Rhein et al., 2013). Oceanic measurement of key biogeochemical variables, which include plankton and nutrients, in general requires ship-based sampling, but good progress is being made on the development of autonomous sensors suitable for deployments on floats, gliders and moorings.

Present observations of the spatial distribution of land-atmosphere $\mathrm{CO}_{2}$ fluxes include the forest inventories made in a number of countries. This method estimates forest above-ground biomass change, and thus cumulative fluxes in the biomass carbon reservoir between two repeated inventories - usually one decade apart from each other. Direct measurements of below-ground biomass are made to support allometric modelling of the relationship between above- and belowground values needed for completing budgets. Another globally distributed type of land-atmosphere $\mathrm{CO}_{2}$ flux observation is from eddy-covariance flux towers. There are roughly 600 flux towers in the worldwide FLUXNET, but coverage is very uneven and cannot presently be exploited to quantify the spatial distribution of regional net $\mathrm{CO}_{2}$ fluxes (due to sampling bias and non-measured disturbance emissions; e.g. Jung et al. 2010). Global time-space resolved estimates of gross primary productivity have been produced by extrapolating flux tower data (Beer et al. 2010). Increasingly, eddycovariance flux towers are also being equipped with sensors to measure the local $\mathrm{CH}_{4}$ fluxes, especially over wetland ecosystems and freshwater.

Small spatio-temporal atmospheric concentration variations of $\mathrm{CO}_{2}$ and $\mathrm{CH}_{4}$ reflect the sourcesink patterns of these gases. An accurate measurement of their concentrations thus allows the quantification of the net large-scale surface-atmosphere $\mathrm{CO}_{2}$ and $\mathrm{CH}_{4}$ fluxes using inverse models of atmospheric transport (section 5.5). However, the density of stations providing suitable in situ data is sparse and does not constrain tropical continental areas, Siberia, the Arctic and under-sampled oceanic regions such as the Southern Ocean.

Several projects for space-based measurements of column $\mathrm{CO}_{2}$ to be used in flux inversions have been developed since the early 2000s, to follow on from the pioneering measurements made by SCIAMACHY from 2002 to 2012. At face value, the largest atmospheric gradients of $\mathrm{CO}_{2}$ related to surface fluxes are observed in the boundary layer, imposing the need for satellite-borne instruments to have a weighting function that can probe the lowermost troposphere (Bréon and Ciais, 2010). For this reason, measurements in the short-wave infrared have been preferred. GOSAT (launched in 
2009) and OCO-2 (launched in 2014) are two spectrometers with high spectral resolution in bands that allow retrieval of column $\mathrm{CO}_{2}$ with an individual precision of 3-4 ppm for GOSAT and about 1 ppm for OCO-2. GOSAT also makes measurements in a thermal infrared band, which supports its provision of data for estimating fluxes of methane. Further discussion of flux estimation is given in section 5.5. Passive optical systems are, however, limited by low solar elevation in what can be provided at high latitudes, particularly in relation to understanding the relationship between permafrost degradation and carbon fluxes.

Complementary to the stations making in situ measurements and the satellite-borne sensors are networks for probing vertical structure, comprising the Total Carbon Column Observing Network (TCCON) of ground-based Fourier Transform Spectrometers that record direct solar spectra in the near-infrared, and the use of commercial aircraft to measure routinely the atmospheric concentration of greenhouse gases, for example by the Comprehensive Observation Network for Trace gases by Airliner (CONTRAIL) and by the aforementioned IAGOS programme. These networks substantially expand the in situ station network and are crucial for the validation of data from present and future satellite systems.

\section{Earth-system modelling}

The current status and prospects for Earth-system modelling are summarized, as a prelude to subsequent discussion of how models help the analysis of observations and how observations help the evaluation of models. The evolution towards applying Earth-system models for environmental monitoring and prediction as well as for climate simulation and projection is outlined. General issues related to the improvement of models, whether through refining the representations of processes that are already incorporated or through adding new processes or components, are discussed. Some important elements of Earth-system models receive further attention.

\section{1 "Seamless" modelling and prediction}

It is expected that the coming ten years will see predictions and scenario-based projections of the Earth system that are increasingly based on fully coupled atmosphere-ocean-land-cryospherebiosphere models at a spatial scale that for the atmosphere will move towards $50 \mathrm{~km}$ for general climate applications and reach around $5 \mathrm{~km}$ for prediction for days and weeks ahead. Progress will require not only attention to developing better representations of the individual resolved and parameterized processes at play in the atmospheric, oceanic, terrestrial, biospheric and cryospheric components of models, but also attention to the interactions between the processes and components. Ensuring that an Earth-system model works well across the range of applications and time scales for which performance can be validated is vital for establishing confidence in the realism of the model and trust in its projections for the future.

The term Earth-system model is often applied to the more comprehensive of the models used to simulate past climate and project future changes, as indicated in section 2.4. However, the models used for global monitoring and for prediction for time ranges of days and upward have also become more comprehensive, and will soon, if not already, also merit being named Earth-system models. ECMWF provides a case in point. It now uses a coupled atmosphere-ocean model for its ensemble weather forecasting throughout the forecast range, and has firm plans to extend this to its highresolution forecasting system, which will soon reach a horizontal resolution of about $10 \mathrm{~km}$. Inclusion 
of a dynamic sea-ice model for predictions at all time-ranges is also under development, having been employed already for ocean reanalysis (Tietsche et al., 2015). Extension of reanalysis to employ coupled data assimilation for atmosphere, ocean and sea-ice has been pioneered in the NOAA/NCEP Climate Forecast System Reanalysis (Saha et al., 2010), and is a major thrust of development at ECMWF (Dee et al., 2014), as discussed further in section 5.4. Furthermore, in developing monitoring and prediction of atmospheric composition as a Copernicus Service, ECMWF has worked with partners to extend its atmospheric model and associated data assimilation to include greenhouse gases and the reactive gases and aerosols that affect air quality and short-term forcing of climate (Hollingsworth et al., 2008). Such activities bring with them the need for more-comprehensive treatments of land-surface processes and representation of ocean biogeochemistry.

Moreover, application models for predicting user-relevant quantities such as energy demand, health impacts or crop yields may increasingly be run by forecasting centres rather than by users, enabling efficient use of the full temporal and spatial resolution of large ensembles of forecasts by taking the application to the data rather than the data to the application. This may be seen as part of an evolution towards the inclusion of such processes within the models themselves, and then inclusion of the feedbacks needed to treat more fully the influences of and impacts on human systems in the models used for environmental prediction and long-term projection.

There is in parallel an increasing level of activity on the part of climate modelling centres to exercise their climate and Earth-system models for experimental predictions. CMIP5 had in its protocol basket a suite of decadal simulations with models initialized at specific times in a hindcast sense. AR5 was the first of the IPCC's reports to assess such simulations performed as an initial-value problem with specified variations in external forcing (Kirtman et al., 2013). The North American Multi-Model Ensemble (NMME) project, initiated in 2011, has focused on seasonal (90-day) predictions, with both operational weather forecasting and climate centres (NCEP, NCAR, NASAGEOS, GFDL and CMC) contributing forecasts every month. These are provided as data to NOAA, and are archived and available to the public. Results that have been analysed include the North American summer of 2012 and winter of 2013-14, and Asian monsoon rainfall. In the USA, the Study of Arctic Environmental Change project has been engaged in forecasting Arctic sea-ice extent, and verifying and documenting the results from a variety of modelling centres and other institutions. Prediction is discussed more fully in section 6 .

These efforts on the part of weather forecasting and climate modelling centres are a realization of the concept of "seamless" prediction and projection basic to the strategy of the WCRP over the period 2005 to 2015, as discussed for example by Palmer et al. (2008), Hurrell et al. (2009) and Hoskins (2013), and illustrated in Figure 7. However, although monitoring, prediction and scenariobased projection over many time ranges will increasingly use what can be regarded as Earth-system models, the various applications will in practice place different emphases on the various model components, generally adopting higher resolution for shorter ranges of prediction and more complex and comprehensive representations of ecosystems for long-term climate projection, for example. Nevertheless, the increased and more diverse use of Earth-system models should be of general benefit, as lessons learnt from performance of models in one type of application may be carried over to the similar models used for other applications. 




Figure 7 Schematic illustrating some of the interactions between various time and space scales in the climate system. Space scales and possible forecasts are indicated. Source: Hurrell et al. (2009).

\subsection{Limitations of models}

As both scientific and public attention shift from the detection and attribution of climate change at the global scale to prediction of its impacts, the limitations of climate models at the regional scale are becoming increasingly exposed. The IPCC AR5 Working Group I (WG I) report illustrates that models often exhibit severe biases at the regional scale, and disagree on the regional response to changing climate forcing, especially for precipitation and for circulation-related features such as the monsoons. Some of this is because of an inherent lack of predictability of internal natural variability that is magnified at the regional scale, but climate models are also generally quite deficient in their simulation of variability, from sub-seasonal out to multi-decadal timescales. This limits the quality of the information that can be provided for adaptation planning and climate services. It also limits the capability of models to be used to attribute extreme events to climate change in a statistical manner. In general, there has been only modest improvement in models in these respects between AR4 and AR5. Many of these model discrepancies are known to result from deficiencies in the representation of atmospheric processes, and there is in general large sensitivity of model simulations to the parameterizations of unresolved processes such as clouds, convection and boundary-layer and gravity-wave drag. Similar sensitivities are seen in weather prediction models, suggesting that shortterm forecasts (for which there is a good statistical basis for evaluation) may provide a route to better understanding of systematic errors in climate models.

These problems in the atmospheric component of Earth-system models have knock-on effects on other components. A number of land-surface processes and variables are highly affected by errors in precipitation in particular. Although increasingly complex models have many potential benefits - 
from increased self-consistency (complete budgets, for example) and information on couplings attention must be paid to the quality of the atmospheric fields that drive so many of the couplings if benefits are to be realized. Work on better understanding and modelling therefore must have one focus directed towards improving core atmospheric elements. Although there is a continuing need for local observations to enhance understanding of the processes that have to be parameterized, extensive data are already available to provide the basis for improved schemes. They include measurements from long-term sites such as those of the Atmospheric Radiation Measurement programme (ARM; Ackerman and Stokes, 2003), from a large number and variety of field studies and from satellite missions such as those on clouds and aerosols flown in the A-train suite noted in section 3.5. Jakob $(2010,2014)$ discusses the challenges and opportunities.

Even at the global scale, both transient and equilibrium climate sensitivity (defined in terms of how global-mean surface temperature responds to a doubling of $\mathrm{CO}_{2}$ ) remains uncertain to something like a factor of two or more (Stocker et al., 2013), mainly due to uncertainties in the cloud feedback. This has not changed fundamentally in the more than three decades that have passed since estimates were published in the so-called "Charney Report" (NRC, 1979). Climate-model projections are increasingly scaled by global-mean surface temperature, so uncertainty in the globalmean surface temperature response has an immediate impact on projections of climate change. The renewed attack being organized by the scientific community on the question of climate sensitivity recognizes that the feedback on circulation is crucial to understanding errors in convective parameterizations (Bony et al., 2013; 2015), and thus is taking more of a global than a local approach. This puts a premium on global observations that can be directly related to atmospheric processes. These may be fairly direct in some cases, such as relating to cloud micro/macro structure or gravity-wave fluxes, while in other cases uncertainties in processes may be most readily detectable in short-term tendencies of other quantities, such as upper-air winds in the case of errors in gravity-wave drag.

Changing dynamics as a result of changing climate forcing remains a significant issue that also needs further study informed by observations. A better ability of models to simulate changes in large-scale circulation patterns should result. Improvement of Earth-system model dynamics is hampered by a number of uncertainties that arise from a combination of uncertain observed trends and a lack of agreement between models on projections for the future. Examples are provided by AR5 (Stocker et al., 2013): "There is low ${ }^{5}$ confidence in near-term projections of a northward shift of $\mathrm{NH}$ storm track and westerlies. There is generally low confidence in basin-scale projections of significant trends in tropical cyclone frequency and intensity in the 21st century. There is low confidence in projections of many aspects of climate phenomena that influence regional climate change, including changes in amplitude and spatial pattern of modes of climate variability."

\subsection{Improvement of models}

Much of the development in modelling has been towards incorporation of additional physical, chemical or biological processes, notwithstanding the challenges of increased complexity noted above. This in turn brings requirements for observations to guide and evaluate progress. Such development includes adding carbon cycles to coupled atmosphere-ocean general circulation models, qualifying them as Earth-system models in the IPCC view discussed in section 2.4. It also

\footnotetext{
${ }^{5}$ As corrected in 2015 (www.climatechange2013.org/images/report/WG1AR5_Errata_17042015.pdf)
} 
includes a more interactive representation of aerosols and adding atmospheric chemistry, as shown in Table 9.1 of Flato et al. (2013) for the models participating in CMIP5 that were evaluated in AR5. Improvements in the sea-ice components included in these models may be inferred from robust evidence that the downward trend in Arctic summer sea-ice extent is better simulated than the time of the AR4. Also in AR5, Church et al. (2013) note substantial progress in ice-sheet modelling, particularly for Greenland. Few Earth-system models include coupled ice-sheet modules, however, and this emerging capability was not used in CMIP5 (Flato et al., 2013). Melting and iceberg calving where ice shelves and outlet glaciers interact with the oceans challenge both observation and modelling.

The other strand of model development has been to improve existing components. Not least is the move towards increased horizontal and vertical resolution, discussed further in the following section. This includes extending atmospheric components upwards into the mesosphere so that models can properly represent the stratosphere, including wave-driven influences on its circulation, the photochemistry of ozone and other trace species, and related radiative effects. It also includes introduction and refinement of multi-layered approaches to modelling ice and lying snow, and downward extension and finer layering in soil models.

Despite progress, the typical horizontal resolutions of current climate models remain insufficient to simulate many of the extremes of interest. Finer resolutions are used in weather prediction, but model topography and parameterizations of convection, clouds and precipitation remain insufficient for accurate simulation and timing of many events. Global models are beginning to be run with sub$10 \mathrm{~km}$ atmospheric grids that resolve mesoscale weather including the most extreme tropical storms, and the coupling of such atmospheric components to fine-resolution oceanic and terrestrial components could revolutionize our ability to correct long-standing model biases, reduce the need for downscaling and provide predictions of regional impacts and changes in extremes from months to decades ahead. However, many errors are not improved solely by increasing resolution and computational costs will limit the extent to which resolution can be beneficially increased on a routine basis within the timeframe of this roadmap and beyond. There will thus remain a need for parameterizations of unresolved and partially resolved processes, and a need to continue to invest in their improvement.

Progress on parameterization is being made and, in particular, the piecemeal approach of the past is being replaced by a more integrated view, such as one that treats the boundary layer, convection and microphysics as essential interactive components rather than treating them separately. This needs to progress a lot further. However, there are measurable improvements in both weather forecasts and simulations of recent climate that can be attributed to parameterization developments:

- improved representation of the boundary layer, clouds and convection, including their diurnal cycles, in models robust across all scales of resolution, including grid lengths of less than $10 \mathrm{~km}$;

- improved understanding of how the representation of land and atmospheric sub-grid scale processes affect the prediction of climate change by these models; 
- improved understanding of the role of clouds, including their micro- and macrophysics, their effects on optical depth and cloud brightness and radiation, and their feedbacks in climate change;

- improved understanding of how clouds and precipitation react to and affect large-scale circulation features of the atmosphere, especially tropical sub-seasonal variability, such as the Madden-Julian Oscillation.

Synergies arise from the increasing use of Earth-system models across all time ranges of prediction and projection discussed in section 4.1. This is notwithstanding the differences in emphasis on resolution and breadth of included processes depending on time range. Use of climate models in initial value problems to make short-term forecasts is exceedingly valuable in revealing problems, and at the same time is essential for seamless forecasts out to decades for various applications. A new focus that has captured the interests of both extended-range weather modellers and climate modellers is the weather-climate interface, from the sub-monthly forecast range upward. This provides the opportunity to confront both weather prediction and climate models with new observational products in innovative analyses and with new diagnostics and metrics of performance. Further discussion of this is given in section 6 . Another development is the extension of reanalyses to the century scale. This requires that much more attention be paid to representing long-term variations in climate forcing in the assimilating models used for reanalysis (Hersbach et al., 2015), which typically come from weather forecasting systems. This should also benefit reanalyses that focus on recent decades, which may underestimate forced changes if the forcings are not represented in the assimilating model, even though the changes are represented to a degree through the observations assimilated.

Various means are being employed to downscale the results of Earth-system models through use of regional models or variable grids. Regional precipitation predictions and projections remain a challenge at all timescales from seasonal forecasting out to centennial climate change. Hall (2014) discusses the issues. There are nevertheless some regions with forecast skill on seasonal timescales, associated mainly with the El Niño Southern Oscillation (ENSO) phenomenon. Emphasis is also being paid to the problem of bias elimination in downscaling techniques. Improving the skill and reliability, and quantifying the uncertainty, of predictions and projections of precipitation at regional scales requires better understanding and model simulation of the teleconnections and drivers of regional climate, such as the changes in the oceans and cryosphere that are relevant to regional precipitation.

There are conceptual and practical challenges in evaluating model results against observations or products derived from them, such as reanalyses. This is a critical topic for models, as the assessment of their performance provides an important basis for their formulations to be enhanced, tested, refined and verified. This is discussed in section 6.2.

\subsection{Spatial resolution}

Improved spatial resolution is still needed for most if not all components of Earth-system models. Discussion often focuses on horizontal resolution, which is a major factor in the computational cost of running a model and for which the benefits of increased resolution in terms of how well a model can represent features of interest is most evident. The paragraphs below are no exception, but it is important to recognize that models also need to represent sharp changes in the vertical, associated 
with thermoclines, the top of the atmospheric boundary layer, cloud tops, the tropopause, sloping fronts and so on. Over the past 35 or so years, the vertical resolution in the free troposphere of ECMWF's highest resolution operational global numerical weather prediction model has increased by a factor of around six, a little under half the factor by which horizontal resolution has been increased. The climate models assessed in AR5 have lower vertical and horizontal resolution than current operational global weather prediction models, but the differences in resolution between the climate and weather-prediction models are generally larger for horizontal than for vertical resolution.

The increasingly fine horizontal resolutions used for global weather prediction now approach or reach around $10 \mathrm{~km}$, and have been one factor in the general improvement of forecasts over time, as seen for example in measures of the skill of circulation-pattern and precipitation forecasts (Dee et al., 2014, Rodwell et al., 2010). These resolutions nevertheless remain inadequate for capturing strong orographically-influenced winds and severe hydrological events such as flash floods. Climate models are generally operated at lower resolutions, these being mostly around $100 \mathrm{~km}$ or coarser for the models assessed in AR5. Such resolutions do not depict well a much wider range of phenomena and extremes, including tropical storms and floods, and the areas worst affected by drought, which are typically of order a few hundred kilometres or less in extent.

It is not only extremes that are affected by limited resolution, however. On land, the barrier effects of mountains can be underestimated by smoothing, allowing air to flow across where this cannot occur in reality. Changes in orographic rainfall can be profound. In the ocean, limited representation of bathymetry can cause some deep trenches to be filled in, atolls and islands not to be depicted with fidelity and ridges not to be properly represented. Because tides and wind move waters across and around these features, mixing within the ocean can be compromised. Heterogeneity of land-surface types has to be taken into account for realistic modelling of land conditions and the fluxes of heat, momentum, moisture and other constituents to and from the atmosphere, with tiling and nesting approaches developed to account for contributions from the various types of surface process that can occur within a model grid cell, including urban effects. Representation is needed also of interactions between land and ocean along coasts and estuaries, which affect marine biogeochemical and ecological systems in particular, but also the physical climate.

Although simulation of a number of features of the atmospheric circulation, including tropical precipitation and circulation, blocking and extratropical cyclones, improves as model horizontal resolution is improved, much of the improvement in the case of the ECMWF model was found by Jung et al. (2012) to come from increasing resolution to $40 \mathrm{~km}$, with relatively small changes for smaller grid lengths. Delworth et al. (2012) show marked improvements in both atmosphere and ocean from a new coupled atmosphere-ocean model with significantly increased resolution.

Ocean modelling in general places particular demands on resolution. This is in part because of specific narrow circulation features such as western boundary currents, which can have distinct downstream effects on atmospheric circulation (e.g. Keeley et al., 2012), and the equatorial Pacific cold tongue, which was singled out by Flato et al. (2013) as an oceanic feature for which models showed particular improvement from AR4 to AR5 (see also section 6.2.6). Fine resolution is also desirable in ocean models because of the important and widespread role played by mesoscale eddy 
motions (with diameters ranging from a few tens to more than $100 \mathrm{~km}$ ), which contain almost $90 \%$ of the total kinetic energy of the ocean and are the major driver of heat transport and interactions with biogeochemistry. Sub-mesoscale processes also need to be taken into account, as they are considered to explain more than $50 \%$ of the vertical velocity field in the upper $500 \mathrm{~m}$ and as such are also important for biological systems and the biogeochemical cycles. The Earth-system and other climate models assessed in AR5 generally have resolutions that do not even resolve the mesoscale, however, and have to rely on parameterizations of the important unresolved dynamics. A finer horizontal resolution of $(1 / 12)^{0}$ is currently used in the ocean analysis and short-range forecasting system of the operational Copernicus Marine Environment Monitoring Service (marine.copernicus.eu).

\subsection{Clouds and aerosols}

As noted above, clouds play a major role in radiative forcing and in the uncertainty associated with climate sensitivity, and models have difficulty in getting the clouds in the right place with the right amount and optical thickness (e.g. Kay et al., 2015). Frequently cloud parameters are tuned in order to achieve an acceptable energy balance at the top of the atmosphere, which may involve brightening clouds to offset deficiencies in amount. The distribution of clouds relates very strongly to the atmospheric circulation, precipitation and convection, while cloud characteristics depend strongly on microphysics that are parameterized. Clouds form because air becomes supersaturated, and supersaturation of air occurs because air rises, or cools for other reasons. There is a strong role of radiation, humidity and atmospheric circulation in controlling cloud processes. As an example, Kay et al. (2015) show that an observationally motivated modification to the shallow convection detrainment increases supercooled cloud liquid, brightens low-level clouds and substantially reduces the chronic bias in Southern Ocean absorbed solar radiation in the NCAR model, with significant impacts on the atmospheric and ocean circulations. Moreover, there is a good possibility of these factors changing in a future climate system in ways that influence cloudiness. The sophistication and complexity of the sub-grid scale parameterizations is increasing over time, and precipitation may become a prognostic variable (e.g. Gettelman et al. 2015).

The representation of the microphysics of mixed phase clouds and the phase partitioning between ice and water clouds in the mixed-phase regime is still not consistent among models nor is the representation of liquid water at mixed-phase temperatures in general consistent with observations (Komurcu et al., 2014). It accordingly remains a subject of model development (e.g. Gettelman et al., 2015). The ability of Earth-system models to predict cloud microphysical properties is limited not only by difficulties in predicting clouds and macrophysical characteristics (Lin et al., 2012) but also by difficulties in determining aerosol characteristics (size and chemical characteristics; Penner et al., 2006) and in simulating aerosol-cloud interactions (Golaz et al., 2013). Prediction of cloud macrophysical characteristics and their response to climate change remains a significant challenge (Sherwood et al., 2014) likely requiring higher-resolution simulations. The treatment of ice nucleation at cirrus temperatures also needs improvement and can lead to large differences in predicted climate forcing (Zhou and Penner, 2014).

Aerosols are increasingly included in models, and are important for their direct effect on solar radiation as well as their interactions with clouds. Aerosol particles deposited on Arctic snow and ice affect albedo and thereby the surface energy budget and timing of seasonal snow-melt (Doherty et 
al., 2010; Dumont et al., 2014). Aerosols also have a number of direct effects on people, including impacts on health and transportation. Dust aerosols are also a source of fertilization of the oceans and distant lands. Aerosols of anthropogenic origin include primary aerosols such as smoke and soot from combustion, secondary inorganic aerosols such as ammonium nitrate and sulphate, and secondary organic aerosols. The processes concerned, including uplift, chemical formation, aging, mixing, transport and deposition, are challenging to model, but important both for short-term forecasting of air quality and for long-term simulation and projection of the state of the Earth system.

The representation of multiphase reactions leading to the formation of secondary organic aerosols is still at an early stage (Lin et al., 2014) but is of primary interest because of coupling to the biosphere and biospheric emissions of volatile organic compounds, which not only vary seasonally, but also as part of climate change (Tawfik et al., 2012; Mao et al., 2013). This also requires that soil moisture and temperature changes be accurately simulated. Understanding of the importance of formation of CCN-sized particles and aerosol nucleation is still under development (Kulmala et al., 2004; Kurtén et al, 2008; Smith et al., 2010; Yu, 2010).

The indirect effect of aerosols on clouds is now being incorporated through more complete microphysics. However, the processes involved are incompletely represented, and indeed incompletely known or understood. Models need to be brought into closer agreement with observations such as those from Cloudsat (Suzuki et al., 2013). Considering for example, the socalled Twomey effect (of brightening when added aerosols increase the number of cloud droplets, thereby redistributing the cloud liquid water over more droplets), models do a relatively good job at simulating drop number at least over the ocean (Quaas et al., 2009) but the relationship between cloud optical depth and liquid water path is too strong. At NCAR, the new microphysics with prognostic precipitation increases the ratio of accretion over autoconversion and appears to significantly reduce aerosol-cloud interactions and indirect radiative effects of anthropogenic aerosols by up to $33 \%$ between simulations with preindustrial (1850) and present-day (2000) aerosol emissions (Gettelman et al., 2015). Better quantification of natural background aerosols is particularly important (Carlslaw et al., 2013).

The CMIP5 forcing from stratospheric sulphate aerosol originating from volcanic eruptions in the pre-satellite era has idealized distributions in space and time. Also, the omission of the cumulative effects of the sequence of relatively small eruptions since the major one of Mt. Pinatubo in 1991 is a minor factor in the failure of models to capture a slowdown in tropospheric warming over recent years (Santer et al., 2014). This brings a need for improved reconstructions for historic eruptions, continued observations for the coming period and appropriate modelling of radiative effects of stratospheric aerosol.

\subsection{Hydrological cycle}

The importance and continuing challenges of modelling the hydrological cycle were introduced in section 2.5.2. Several problems in the modelling of precipitation in particular were mentioned both there and in discussing model resolution in section 4.4. The difficulty that current climate models have in simulating the water cycle in turn affects the representation of the carbon cycle, especially through the modelled health and growth of vegetation. In addition, effective incorporation of fully 
integrated atmospheric chemistry depends on realistic modelling of cloud and precipitation processes, as briefly discussed in the following section.

Improving the representation of precipitation in models depends greatly on the atmospheric circulation and processes discussed in section 4.5 , and in part on the representation of the local processes involved in its formation and fall. The representation of precipitation characteristics in several models was described as "dreary" by Stephens et al. (2010) based on comparison with observations over the oceans from CloudSat. Convection is often triggered prematurely in models, too early in the diurnal cycle (Trenberth et al., 2003). Bechtold et al. (2014) show how the scheme used at ECMWF benefits from introducing a new convective closure that improves the predicted spatial distribution and local intensity of convection as well as its diurnal cycle. The paper serves also as an example of some of the other sources of data that may be used to evaluate precipitation from global models: from active (radar) and passive microwave instruments, from infrared imagers in geostationary orbit and microwave sounders in polar orbit, from ground-based radar and rain-gauge data, and from a cloud-resolving model run over a regional domain.

The representation of circulation features on a range of scales is determined by a number of processes, among them the latent heating (and cooling) that is associated with the formation (and evaporation) of precipitation itself. Features such as the intertropical convergence zones, monsoons, tropical and extratropical cyclones, jet streams and associated storm tracks, and more, must be represented well. Accompanying distributions of water vapour, dependent also on the representations of evaporation and transpiration, must also be free from significant error. This is more challenging for longer time ranges of prediction and for climate simulation and projection, as assimilation of observational data provides a control on circulation and water vapour in integrated analyses and the short-range forecasts initialized from them. Flato et al. (2013) document the status of climate modelling in these regards, identifying a number of substantial shortfalls in performance where progress in recent years has been slow.

Global-mean precipitation is influenced also by the ability of the atmosphere to disperse the latent energy released when precipitation forms. Heat generally is moved around and ultimately radiated to space. The long-wave radiation involved is also modified by the increase in atmospheric water vapour that is well established as accompanying warming of the ocean-surface and troposphere, with the exception of the near-surface over land. The radiation change limits the fractional rate of increase of precipitation to be less than that of water vapour (Stephens and $\mathrm{Hu}$, 2010), and evaporation has to change similarly in the global mean on annual and longer time scales (Trenberth, 2011). Modelling the quantitative precipitation response to increasing $\mathrm{CO}_{2}$ evidently poses difficulty. Pendergrass and Hartmann (2014) show that the CMIP5 models are robust in having a much smaller rate of increase in precipitation than water vapour, but that they differ by a factor of two in their precipitation increases, a finding attributed to their different changes in clear-sky radiative cooling.

There are also needs and opportunities for better modelling of the land-surface component of the hydrological cycle. As with integrated Earth-system modelling at large, this partly involves improving the representation of processes already included, such as those related to soil moisture, snow cover and melt, surface and deep water storage, and run-off. It also involves adding processes new to most models, such as irrigation, water management, land management and land-use change. 
More-refined precipitation downscaling would benefit a number of application models linked to Earth-system models, including river-routing models used in short-term flood forecasting and in assessing changes in river flow within a changing climate. It may also become beneficial for Earthsystem models to be run with land-surface components that operate at finer horizontal resolution than their atmospheric components (Balsamo et al., 2014). Freshwater and nutrient flow into the oceans from rivers and stomatal changes and reduced transpiration due to increased $\mathrm{CO}_{2}$ are examples of interactions with other components of the Earth system that need to be modelled.

\subsection{Atmospheric chemistry}

Incorporation of atmospheric chemistry in Earth-system models is important for short-term airquality forecasting, for assessing the regulation of emissions, proposed or actual, and for modelling climate forcing and the impacts of climate change on air quality. Although relatively simple and computationally undemanding approaches have proved useful in modelling stratospheric ozone and methane (e.g. Cariolle and Déqué, 1986; Monge-Sanz et al., 2013), and are currently used, for example, by ECMWF in operational weather forecasting and reanalysis, comprehensive schemes are required to represent tropospheric chemistry or for better treatment of the stratosphere. Chemical transport models in which meteorological variables are prescribed are commonly used for shortterm air-quality forecasting and for understanding longer-term changes, but the direct inclusion of comprehensive chemistry within Earth-system models has a number of advantages. Eleven of the CMIP5 models whose results were considered in AR5 (Table 9.1, Flato et al., 2013) were categorized as including atmospheric chemistry, either directly as part of their atmospheric component or as a separate coupled component. Four of these models also included ocean and land biogeochemistry.

The advantages of fully integrating chemistry within the atmospheric component of a model include use of (i) the model's parameterizations of convection and diffusion to redistribute species, (ii) the convection scheme also to parameterize nitric oxide generation by lightning, (iii) model cloud and aerosol distributions in calculating photodissociation and heterogeneous reactions, (iv) the model's precipitation parameterization in the calculation of wet deposition and $(v)$ the model's landsurface parameters in the calculation of dry deposition and biogenic emissions. An integrated scheme is used, for example, in the version of the ECMWF model enhanced to provide the operational global Copernicus services for atmospheric composition (Flemming et al., 2015).

Chemical schemes vary in terms of the number of species and reactions included, and the associated computational costs. The latter are generally high, however, and incorporating comprehensive chemistry may increase the net cost of a model by an order of magnitude unless offset by running the model (or at least its chemistry) at around half the horizontal resolution than would otherwise be possible. Use of such models in data assimilation and short-range forecasting offers an opportunity for comparing, choosing and improving schemes on the basis of direct comparison with observations of chemical species at a time when other components of the model are generally at their most realistic. The performance of sixteen models with a wide range of resolutions, chemical schemes and reactions with clouds and radiation has been compared for longterm simulations in the Atmospheric Chemistry and Climate Model Intercomparison Project (Lamarque et al., 2013). Both approaches are needed; this is an area where the synergies discussed in section 4.3 of using and evaluating models for a range of applications may be of particular benefit. 


\subsection{Land biogeochemistry}

If anthropogenic release of $\mathrm{CO}_{2}$ into the atmosphere were to cease, natural biogeochemical processes would remove most of the $\mathrm{CO}_{2}$ of prior anthropogenic origin from the atmosphere on a multi-millennial time scale. Natural removal processes are, however, less powerful on the decadal scale most immediately relevant to climate policy. A newly developed metric appropriate for this time scale, the transient climate response to cumulative carbon emissions (TCRE), is highly policy relevant as it directly links net anthropogenic $\mathrm{CO}_{2}$ emissions to the change in global-mean surface temperature (Allen et al., 2009; Gillett et al., 2013). TCRE is one of the few really new conceptual developments from AR4 to AR5, but the usefulness of the concept is hindered by a large quantitative uncertainty, to which the carbon cycle response to environmental changes is a key contributor.

Through several CMIP phases there has been systematic and demonstrable progress in physical climate simulation at both whole-system and process level. Climatologies, modes of variability and historical trends are now better modelled and better understood than before, notwithstanding continuing needs for improvement and slow progress in some areas. Earth-system models are at a crucial stage where systematic and coordinated evaluation and development of the biogeochemical components and their physical counterparts must be kept in pace for climate applications.

Several specific processes are highlighted in the following sub-sections. Whilst there are many important processes and ways of prioritising, the focus here is on those of importance at a global climate scale, and on decadal-to-century timescales. Table 6.10 from the AR5 contribution of Ciais et al. (2013) shows clearly how different processes and sensitivities dominate at different timescales, highlighting the importance of careful application of evaluation on a range of timescales.

\subsubsection{Nitrogen cycle}

Interactions between the carbon and nitrogen cycles attenuate the carbon cycle response to both $\mathrm{CO}_{2}$ and the state of the climate system in general (Zaehle and Dalmonech, 2011). Anthropogenic reactive nitrogen deposition also has important consequences for the climate system through feedbacks on atmospheric $\mathrm{CO}_{2}$ and $\mathrm{N}_{2} \mathrm{O}$ (Zaehle et al., 2011). Hence the terrestrial nitrogen cycle acts as both a key forcing and a feedback for the terrestrial carbon cycle. Only one of the land-surface schemes used in the CMIP5 models, adopted in two of the Earth-system models, included an interactive nitrogen cycle.

AR5 concluded with high confidence that low nitrogen availability will limit carbon storage on land (Fig. 6.35, Ciais et al., 2013), even when considering anthropogenic nitrogen deposition. It was estimated that omission of the process in most CMIP5 models led to an overestimation of as much as 100-200 PgC of terrestrial carbon uptake by 2100 across all scenarios. Inclusion of the nitrogen cycle should then lead to a higher TCRE than the AR5 estimate. But the magnitude is uncertain because the net effect of nitrogen is a fine balance between several processes, which makes its evaluation crucial.

\subsubsection{Permafrost, wetlands and methane}

Although it was concluded in AR5 (Collins et al., 2013) that a retreat of permafrost extent with rising global temperature is virtually certain, Ciais et al. (2013) assessed with only low confidence the range of future methane emission to the atmosphere from thawing permafrost (50 to $250 \mathrm{PgC}$ 
released under RCP8.5). This lack of confidence in the quantification of permafrost carbon release is in large part due to the very large difference among models in their present-day permafrost extent (Koven et al., 2013). Improving modelling and evaluation of permafrost physics is a crucial step to improving Earth-system models.

Emissions from existing wetlands also have the potential to increase terrestrial emissions of methane to the atmosphere, and again the processes concerned are highlighted by AR5 as having high uncertainty and low confidence. There is currently a model consensus that future wetland emissions are likely to increase due to the direct effect of $\mathrm{CO}_{2}$ fertilization, but there is a large uncertainty in the response of wetland $\mathrm{CH}_{4}$ emissions to climate as well as in the mechanisms driving future changes. Again, physical simulation of wetland extent is a crucial factor. Ciais et al. (2013) note that uncertainty in modelled wetland emissions exists in particular because there are limited observational data sets available for model calibration and evaluation.

\subsubsection{Fire}

Climate simulations using fire models indicate spatially variable responses in fire activity to future climate change depending on changes in moisture and fuel availability, as well as on anthropogenic activities. It may be expected that fire frequency will increase in boreal forests, Mediterranean and other dry regions, while changes in the tropics are less certain (Ciais et al., 2013). About half of the Earth-systems models that participated in CMIP5 included fire modules, but detailed evaluation of these modules and analysis of the fire projections has yet to be undertaken. Development and evaluation of fire components in climate applications is clearly a high priority as fires have impacts on vegetation dynamics, the carbon cycle and atmospheric composition. Fire modelling also needs to be developed further for application in fire data assimilation and air-quality forecasting systems.

\subsubsection{Land use, land management and land cover change}

The terrestrial carbon cycle is heavily perturbed by direct human disturbances through deforestation, agriculture and a multitude of management practices, in the areas of forestry, fire management and irrigation, for example. Almost $40 \%$ of ice-free land areas are already used for agriculture and pasture (Foley et al., 2011), and less than a quarter of the world's forests can still be considered near-natural. Land use and land cover change (LULCC) exerted dominant control on the land carbon storage in the 19th and 20th centuries, and will be important into the future. For the first time, CMIP5 scenarios and models included LULCC as an external forcing affecting both the physical system (e.g. albedo) and the carbon cycle ( $\mathrm{CO}_{2}$ emissions). This had been missing in the earlier CMIP3 simulations that contributed to AR4. However, the CMIP5 experiments have been of limited value to date, due to large differences among models in implementation of LULCC processes, an absence of common terminology and the lack of model evaluation. Similarly, LULCC could not be included in ECMWF's first century-scale reanalysis because of significant local differences for recent decades between the CMIP5 fields and those established for use in the ECMWF model, and because change information was not provided for some of the model variables (Hersbach et al., 2015). Furthermore, a representation of the various land management practices is completely lacking in current Earth-system models.

Modelling the dynamics of unmanaged vegetation systems is one requirement for comprehensive study of long-term climate variability and change. Interactions with other modelled 
components also come into play. For example, canopy radiation schemes are important for estimating surface carbon, energy and water fluxes: Loew et al. (2014) showed that there are considerable systematic differences between the simplified radiative schemes in current use, and demonstrated a physical inconsistency when separated approaches are used for simulating absorbed and reflected fluxes. Implementing a consistent approach should also allow a better assimilation of space-based data products.

\subsection{Ocean biogeochemistry}

Including ocean biogeochemistry in an Earth-system model is important for much more than ensuring that the oceanic uptake of carbon dioxide from the atmosphere is represented well enough for the future amount of carbon dioxide in the atmosphere and associated climate change to be estimated reliably for a given scenario concerning emissions. Changes in the chemical properties of the ocean have a large impact on ocean health and productivity: the upwelling zones of the oceans provide nutrients that support some of the most biologically productive zones of the planet, and there is growing evidence that physical and chemical changes in the ocean strongly control ocean ecosystems. For instance, changes in ocean stratification can influence the availability of nutrients in the photic zone, and also influence the occurrence of de-oxygenated, or 'dead', zones. Ocean acidification also has the potential to have far reaching effects on the health of ocean ecosystems. Warmer waters can cause coral bleaching. Modelling changes in the biogeochemical system and in marine ecosystems, supported by observation, is critical to projecting their future states, as well as the oceans' capacity to provide food.

Half the Earth-system models participating in CMIP5 and evaluated by Flato et al. (2013) in AR5 were reported to include ocean biogeochemistry. This is two fewer than were reported to include terrestrial carbon processes. Flato et al. note that around half of the models that did include ocean biogeochemistry used schemes that partitioned marine ecosystems into nutrients, phytoplankton, zooplankton and detritus, while others used more simple schemes. Newer models also included parameterizations of the production of calcium carbonate. Some models included a sediment layer. A recent evaluation of six of the CMIP5 models with more advanced ocean biogeochemical representations is reported by Nevison et al. (2015). Each of the evaluated models took into consideration the availability of the nutrient elements nitrogen, phosphorus, silicon and iron. Four represented at least two classes of phytoplankton size, and three explicitly modelled nitrogen-fixing diazotrophic plankton. The evaluation involved directly comparing the Earth-system model outputs with ocean-colour data from satelllites, and comparing in situ atmospheric constituent measurements with atmospheric transport models driven by the oxygen, nitrogen and carbon dioxide air-sea fluxes from the Earth-system models. Three of the Earth-system models emerged from the latter test as leading to reasonable agreement with the atmospheric data, within the uncertainty of the atmospheric transport modelling. 


\section{Earth-system data assimilation}

Data assimilation is discussed not only because it utilizes observations to generate datasets for monitoring the Earth system and for initiating and evaluating predictions, in particular through reanalysis, but also because of the feedback it provides on the quality of both the models and the observations used in the process. Inverse methods for surface-flux or model-parameter estimation are also discussed.

\subsection{Introduction}

Data assimilation offers a fundamental approach for estimating successive instantaneous states of the Earth system or of its components. A forecast carries information from past observations forward in time to provide a background estimate of the state. The latest observations are then used to refine the estimate. This is used in turn to initiate the next background forecast in the dataassimilation sequence, and forecasts for longer time ranges when needed. In addition to carrying forward information from past observations, the background forecast spreads this information in space and from one variable to another. Observations of one atmospheric chemical variable may, for example, influence the value of an unobserved variable through the modelled chemical interactions between the variables in the background forecast. The background also incorporates the knowledge of the Earth system that is built into the model used to make the background forecast. Knowledge of the uncertainties in observations and forecasts is incorporated in the observational and background error variances and covariances that influence the weight given to prior and current information. This too can spread information in space and from one variable to another. For example, an observation of atmospheric temperature will typically change the analysis not only of temperature but also of wind, to keep the atmospheric state close to thermal-wind balance.

Data assimilation is particularly suited to integrating the information from a mix of observing systems of different accuracies and coverage. Some space-based observations may be used particularly effectively by assimilating them in a form closely related to the original measurement, radiances for example, as the background and other assimilated data aid the extraction of the information on the geophysical state that these observations contain. Joint assimilation of multiple types of observation also provides a basis for estimating biases in the data from particular instruments (Dee, 2005; section 5.5), providing an alternative or complement to the calibration activities of space agencies, such as undertaken for GSICS. The assimilation process also provides feedback on other aspects of the quality of observations, as the background may agree better with data from one type of measurement than another, and on the quality of the assimilating model, as when background fits to several types of observations vary similarly over time, a modelling problem may be the cause. Simmons et al. (2014) and Simmons and Poli (2015) provide recent discussion and examples from atmospheric reanalysis.

Data assimilation may also contribute to assessment of background-model components that are not directly constrained by observations. It enables the performance of such model components to be assessed against independent observations with limited influence of error from those interacting model components that are constrained by assimilated observations. This is in contrast with evaluation of free runs of a model in climate simulation, when error in one component of the model may be responsible for error in another component, however well the latter is formulated. 


\subsection{Data assimilation for specific components of the Earth system}

Assimilation of data for the atmosphere is very well established for operational numerical weather prediction, where improvements in methods allied with better modelling, both enabled by increases in computing capacity, have contributed predominantly to the improvement of forecasts since the observing system was improved very substantially in the late 1970s (Simmons and Hollingsworth, 2002; Dee et al., 2014). In particular, the introduction of variational methods in the 1990 s was key to realising much of the potential of radiance measurements from space.

Complementary or alternative ensemble approaches have become important for characterising uncertainty in analyses and background forecasts, thereby enabling better use of observations in general. Further algorithmic improvement in this area and increased utilization of satellite data influenced by cloud, precipitation and the underlying surface are likely to be significant topics of development for the next decade. The impacts of the various types of atmospheric observation are quite well understood for weather prediction (WMO, 2012). Overall design of the atmospheric observing system nevertheless has to take other applications into account, not least those related to the longer timescales involved in climate variability and change.

Use of data assimilation is also established for atmospheric models that include trace gases and aerosols, for example for forecasting air quality or monitoring the long-range transport of pollutants. This may be achieved using transport models in which the meteorological fields are prescribed from either reanalysis or operational numerical weather prediction, but the global system developed under the Copernicus initiative couples the meteorological and constituent data assimilation (section 5.4). This system provides boundary values for regional models, and enables both forecasting and retrospective assessment of air quality for the European domain. This has been developed from the system described by Hollingsworth et al. (2008) and uses an ensemble of regional transport models, all of which now include assimilation of data on trace-gas and aerosol species. The global system is also applied to produce analyses of carbon dioxide (Agusti-Panareda et al., 2014) and methane (Massart et al., 2014).

Data assimilation is also used routinely for ocean forecasting and reanalysis. Its integration of different streams of observations (discussed in section 3.6.2) to provide values for both those model variables that are directly observed and those that are not is particularly important for the ocean, where sub-surface observations are sparse, aside from Argo temperature and salinity data above $2000 \mathrm{~m}$ for the past decade (Figure 6), and satellites sense only the surface and some bulk properties. Observations such as those of sea-level anomalies from altimetry have to be used to correct both temperature and salinity in such a way as to maintain dynamical balance. Atmospheric observations play a key role also, usually through being used in atmospheric data assimilation, which commonly provides the surface fluxes used to drive assimilating ocean models. In this regard, the important momentum fluxes are regarded as a relative strength of atmospheric analyses; corresponding fresh water and solar radiative fluxes are of poorer quality. Overall, there is evidence that current data assimilation systems are not exploiting the full potential of the observations; challenging areas are the western boundary currents and equatorial regions, where the information provided by observations is quickly lost in ensuing forecasts. Use of the information on the geoid and bottom pressure from gravity missions needs to be developed further.

Much of the use of SST data in assimilation systems is via gridded analysis products, although use may be made of SST retrievals along orbital swaths (so-called level-2 products) along with in situ SST 
data. The use of gridded products has some known problems, notably in ensuring consistency with sea-ice concentration in marginal zones. There are also issues of consistency between near-real-time products such as provided by the GHRSST project (www.ghrsst.org) and long historical SST reconstructions. This is an issue to be addressed for consistent initialization of seasonal and decadal forecasts, and for near-real-time extensions of reanalyses. The SST data used in ocean assimilation may also be inconsistent with that used in the atmospheric reanalysis or numerical weather prediction (NWP) system that provides the surface forcing. Such issues provide motivation for direct assimilation of SST and sea-ice observations in coupled systems.

Quantification and reduction of the uncertainty of ocean analyses is difficult due to the sparsity of observations. Independent data on ocean currents from moorings have proved useful for identifying and correcting observational bias (Bell et al., 2004; Balmaseda et al., 2007) and for developing constraints to ensure balance of current and density increments in data assimilation (Burgers et al., 2002). Sea-level measurements from tide gauges also provide valuable independent information, with the added benefit that some span long periods of time, and thus are particularly important for evaluating the quality of ocean reanalyses prior to the satellite era (Chepurin et al., 2014).

Altimeter data as detailed in section 3.6.2.4 have been assimilated operationally at ECMWF for more than twenty years to determine the sea state, or more specifically a discretized representation of the direction and spectrum of the height of ocean surface waves. The method used from the outset has been optimal interpolation (Lionello et al., 1992; updated as described in system documentation available from www.ecmwf.int). Again, analysis quality is also influenced by the atmospheric data assimilation system that provides the driving of the wave model.

Assimilation of ocean colour data products is being developed to enhance understanding and monitoring of marine biogeochemistry. Examples include assimilation into global (Ford et al., 2012), regional (Fontana et al., 2013) and shelf (Ciavatta et al., 2014) models that couple ocean physics and biogeochemistry. Coordinated efforts include those undertaken as part of the development of the Copernicus Marine Environment Monitoring Service and within the ESA CCI, and with a data record that goes back to 1997 and is set to be sustained into the future (section 3.6.2.7) there is good reason to expect progress to be continued.

Data assimilation is used routinely in global weather prediction and reanalysis systems for a number of land variables that are coupled with atmospheric variables in these systems. Screen-level observations of temperature and humidity have been used for some time, and with some success, to constrain soil temperature and humidity (e.g. Albergel et al., 2012; 2015) while more recently surface soil moisture data derived from the ASCAT scatterometer have also been assimilated, using a nudging scheme (Dharssi et al., 2011) or an ensemble Kalman filter (de Rosnay et al., 2013). Further developments in soil-moisture analysis include the direct assimilation of passive microwave radiances from SMOS (Muñoz-Sabater, 2015) and assimilation of downscaled SMOS retrievals in a catchment model (Ridler et al., 2014). Comparative evaluation of SMOS and AMSR-E soil moisture information in a data assimilation context is reported by Al-Yaari et al. (2014). In situ snow-depth measurements augmented by snow cover estimated from satellite data are assimilated to produce snow-depth analyses (e.g. de Rosnay et al., 2014). A fire assimilation system using radiative power observations from space is also run routinely in the Copernicus system (Kaiser et al., 2012). 
Development work at ECMWF includes study of the assimilation of data on surface albedo and leaf area index from space-based measurement (Boussetta et al., 2015).

In carbon cycle data assimilation systems (CCDASs; e.g. Kaminski et al., 2013), the parameters of a mechanistic surface flux model are optimized. The model is then used to calculate the fluxes and their uncertainties. This approach provides the opportunity to use additional observational data streams consistently to constrain the model (e.g. FAPAR and terrestrial or ocean local exchange flux measurements (Rödenbeck et al., 2014)). The development of CCDAS systems of varying complexity from simple diagnostic flux schemes to complete land and ocean surface modules of Earth-system models currently constitutes an area of very active research. For surface-flux estimation it provides an alternative approach to the use of inversion methods discussed below in section 5.5.

Other examples of data assimilation include the use of soil-moisture data by Brocca et al. (2010) and streamflow data by McMillan et al. (2013) in catchment-scale models of river flow. Ecological applications related to outbreaks of infectious diseases and the assessment and prediction of fish stocks are discussed by Niu et al. (2014). Ines et al. (2013) and Jiang et al. (2014) report studies in which leaf area index data are assimilated directly into crop and general vegetation models.

\subsection{Reanalysis}

Reanalysis applies data assimilation to time series of past observations to estimate the long-term state of the modelled system. It is particularly well established, with many users, for the atmosphere, for which the latest to be completed using multiple types of observation are JRA-55, produced by the Japan Meteorological Agency for the period from 1958 (Kobayashi et al., 2015), and NASA's MERRA-2, from 1979 onwards. This class of reanalysis typically utilizes all types of upper-air and surface data that can readily be assimilated, and extends back either to the time radiosonde data became widely established in the northern hemisphere or globally, or satellite data likewise became comprehensively established. Atmospheric reanalyses extending back over a century or more that assimilate only surface atmospheric observations have been pioneered by Compo et al. (2011). Other observations are used implicitly in such reanalyses, through specified sea-surface temperatures, sea-ice cover and temporal distributions of radiatively active atmospheric constituents, and may be extended to include changing surface characteristics. Insights into the performance of the data assimilation and the prescribed forcings may be gained from corresponding simulations that omit the assimilation of surface atmospheric observations. Hersbach et al. (2015) provide a recent example. Such simulations also form the "AMIP" component of CMIP5, evolving from the original Atmospheric Model Intercomparison Project (Gates, 1992).

Reanalysis has become important also for the oceans, for purposes such as monitoring, forecast calibration and understanding the role of the ocean in the Earth system, addressing for example the key issue for climate variability and change of the extent to which heating of the oceans is distributed between upper and deeper layers (Balmaseda et al., 2013b). Improved land-surface products based on the MERRA (Rienecker et al., 2011) and ERA-Interim (Dee et al., 2011) reanalyses have been derived respectively by Reichle et al. (2011) and Balsamo et al. (2015), through running updated land-surface model components driven by reanalysed meteorological fields, with precipitation rescaled to match independent monthly analyses of rain-gauge and other observed data. Significant developments are likely in this area during the roadmap period, as such approaches readily lend themselves to running the land-surface model at higher resolution, including carbon- 
cycle components and implementing dedicated data assimilation for an increasing range of land and near-surface atmospheric variables (Balsamo et al., 2014).

Changes over time in the types, coverage and biases of observations pose the key dataassimilation challenge for reanalysis, especially in the presence of background-model error. When such changes in the observing system coincide with real changes in climate variability, true and spurious signals in reanalyses can be difficult to disentangle. This is the case for the intensification of tropical Pacific winds after the 1998-1999 La Niña event that is thought to have led to an increase in the heat absorption by the ocean, and the slowdown in global surface warming. Studies of this have made substantial use of data from reanalyses, but these reanalyses are prone to various degrees to exhibit spurious shifts in some of their products in the late 1990s associated with the introduction of the AMSU-A microwave sounding instrument on the series of operational meteorological polarorbiting satellites. Establishing whether changes identified from reanalysis products are robust is vital for the understanding of climate. Boisséson et al. (2014) provide a recent example, demonstrating reliability of ERA products for tropical Pacific winds in the 1990s and 2000s.

Observing-system changes have been a particular problem for hydrological variables in past atmospheric reanalyses, which have exhibited a number of large spurious changes over time. It is seen most directly in examination of the hydrological budget itself, as shown for example by Trenberth et al. (2011), but associated changes in latent heating and the circulation it drives can result in degraded fits to temperature observations, remotely as well as locally (Simmons et al., 2014). Nevertheless, the newer reanalyses generally perform better in their treatment of key aspects of the hydrological cycle (Bosilovich et al., 2011; Trenberth and Fasullo, 2013; Kobayashi et al., 2015). Although these and other studies show that problems remain, there are reasons to expect continued progress over the next decade, through recovery and reprocessing of past observations, through the new data provided by the GPM constellation of satellites, and through continued attention to modelling and data-assimilation issues.

\subsection{Coupled data assimilation}

Coupled data assimilation for the atmosphere, land and sea state is established for weather prediction and reanalysis, in the sense that although analyses themselves are generally performed separately for the three domains, the background-forecast model couples the domains, enabling observations of one domain to influence the subsequent analysis of other domains. In addition, differences between screen-level atmospheric observations and background estimates from the atmospheric model may serve as an input to the land-surface analysis (de Rosnay et al., 2013).

Coupled assimilation approaches for atmosphere and ocean and for chemical composition within the atmospheric component have been topics of recent development. Together with a broadening of the range of analysed terrestrial variables and development of assimilation of ocean-colour data into ocean models that include biogeochemistry (section 5.2), the elements for coupled Earthsystem data assimilation for forecasting and reanalysis applications are being put in place, and substantial further progress is expected over the roadmap period. Tighter forms of coupling are also expected, even though systems are likely to make only limited use within the analysis steps of multivariate relationships that span the domains. 
A particular focus has been on the coupling of atmospheric and oceanic systems, with a view to improving all time ranges of forecasting and reanalysis. Examples include:

- Development of a coupled Ensemble Kalman Filter system at GFDL by Zhang et al. (2007). The system generates realizations of initial states (and state estimates) of the coupled system that are consistent with observations. This was tested with promising results in a perfectmodel setting, using realistic simulated oceanic observations, but monthly-mean analysed data for the atmosphere. It is currently used with a new higher resolution atmosphere/land model in the Forecast-oriented Low-Ocean Resolution system (Jia et al., 2014; Vecchi et al., 2014) that contributes to the NMME, although initial atmosphere/land conditions are to date determined only from an AMIP-style simulation rather than from assimilation of atmospheric observations.

- Demonstration by Fujii et al., (2009) of a better spatial distribution and variability of tropical precipitation in a coupled system in which ocean data alone were assimilated than in an AMIP-style simulation using the atmospheric model and separately analysed SST. Negative feedback between SST and atmospheric convective activity, not properly represented in the AMIP run, was shown to improve the representation of the atmospheric circulation as well as precipitation in the coupled assimilation.

- Use of a coupled system in the NOAA/NCEP Climate Forecast System Reanalysis (Saha et al., 2010). Separate atmospheric and ocean analyses were carried out, with atmosphere and ocean coupled in the model used for the background forecast. SST was prescribed for the atmospheric model, using a separately produced analysis, and the upper level of the ocean model was relaxed to this SST as part of the ocean analysis.

- A scheme at ECMWF that introduces a degree of coupling within the analysis itself (Laloyaux et al., 2015), building on the incremental four-dimensional variational (4D-Var) approach established for the atmosphere (Courtier et al. 1994). The analysis is produced iteratively using successive linearizations of the model and operators that map model variables to the quantity that is measured. Each iteration begins with an integration of the coupled model forward in time, over the length of the analysis window. The variational problem is linearized about this reference trajectory and then solved separately for the atmospheric and oceanic components of the system. From the updated state the coupled model is again integrated to generate a new reference trajectory, which is then used for the next iteration of the scheme. Observations of SST are not assimilated; instead the temperature of the uppermost ocean is relaxed towards an external SST analysis, as in the NOAA/NCEP system. Land-surface and seastate analyses are produced in separate steps and feed into the next background forecast, although they too are candidates for tighter coupling. The focus at ECMWF is initially on the application to reanalysis (Dee at al., 2014), but development for medium- and longer-range prediction is within ECMWF's four-year planning horizon.

An envisaged future development is to determine the SST in such coupled data assimilation systems directly by assimilation of space-based and in situ observations, rather than through use of a separately derived product. A desirable further development is the formulation of error covariances between the variables involved in the air-sea interaction. The required balance relationships between variations in the ocean mixed layer and in the atmospheric boundary layer can be obtained 
from model integrations, but verifying data are needed. Collocated oceanic and atmospheric observations from the tropical moored buoys are expected to be an important source of information in this regard.

Coupling of meteorology and chemistry within the system developed by ECMWF and its Copernicus partners (Hollingsworth et al., 2008) utilizes the iterative 4D-Var scheme in a way similar to that for coupled atmosphere/ocean assimilation discussed above, with separate analyses for several gaseous and aerosol species coupled at the level of the reference trajectory. The system has been used for two reanalyses covering periods from 2003 during which plentiful data from the EOS and Envisat missions were available. Inness et al. (2013) report on the evaluation of the reactive-gas component of the more recent of these reanalyses, while Bellouin et al. (2013) discuss its use for estimating aerosol radiative forcing. However, although observations of constituents carry implicit information on the winds that advect the constituents and on the temperature and cloud that influence them through reaction rates, it remains a challenge to extract this information, and to date the adjustment of meteorological variables due to observations of chemical species has been suppressed, as discussed by Dee at al. (2014). Relaxation of this constraint, fuller interaction within the model of aerosols, fast-reacting gases and long-lived greenhouse gases, building on the recent in-lining of chemistry (Flemming et al., 2015), and coupling with the carbon component developed for the land-surface model (Boussetta et al., 2013b) should occur over the roadmap period.

\subsection{Parameter estimation and flux inversion}

Data assimilation methods are also used to estimate parameters or variables other than, or as well as, the state variables used for initialising forecasts or monitoring variability and change. Although most of the examples discussed below use models of limited complexity or domain, these models may be driven by data from more comprehensive models, and many of the resulting estimates are relevant to the operation, validation or interpretation of the results of integrated Earth-system models.

Biases of the assimilated observations may be estimated concurrently with the estimation of prognostic model variables. Developed initially by Derber and Wu (1998) for adjusting the bias of satellite radiance measurements for use in numerical weather prediction, it entails establishing a linear predictor model for the bias and adding parameters of this model to the set of model variables that are determined by the minimization process at the heart of the variational scheme. Application to radiance bias adjustment in reanalysis is discussed by Dee and Uppala (2009) and Simmons et al. (2014) in the context of the representation of low-frequency variability and trends in temperature and moisture in ERA-Interim and other atmospheric reanalyses that span the lifetimes of many sets of radiance measurements. The variational method is increasingly also being applied to adjust other types of observation that are subject to significant bias.

Parameters of a model may be refined by adjusting them variationally to obtain a better fit to observations. For example, Kuppel et al. (2012) optimized 21 parameters of a vegetation model to improve model fits to data on fluxes of carbon dioxide and water from 12 tower sites in deciduous broadleaf forests, using local meteorological data from the towers to force the model. Running the model globally with meteorological forcing from ERA-Interim showed that use of the optimized parameters improved the fit to independent vegetation index data from MODIS. 
Estimation of net sources and sinks of carbon dioxide through inversion utilizing surface measurements of gas concentrations dates back to the 1980s. The Carbon Tracker website (www.esrl.noaa.gov/gmd/ccgg/carbontracker; Peters et al., 2007) provides the results from one particular inversion system, together with substantial supporting information. Peylin et al. (2013) compared fluxes from two versions of Carbon Tracker and nine other systems, several of which covered periods of more than twenty years. Chevallier et al. (2011) showed encouraging comparison with flux estimates from a much smaller number of TCCON stations making total-column measurements. Basu et al. (2013) and Maksyutov et al. (2013) present first estimates of surface fluxes derived from total-column retrievals of data from GOSAT. Used alone in inversions the GOSAT data give results consistent with but not superior to those from the surface networks, but they have significant impact on flux estimates for the tropics and southern extratropics when used together with the surface data. Using the resulting fluxes in model runs improves the fit to TCCON data in the northern extratropics, but the presence of biases in the GOSAT retrievals remains an issue. Reuter et al. (2014) discuss a stronger than expected biospheric sink for $\mathrm{CO}_{2}$ in Europe using data from SCIAMACHY and GOSAT.

Use of satellite data to estimate fluxes of methane is longer established than for carbon dioxide. Estimates of about ten-year duration using retrievals from SCIAMACHY together with in situ surface measurements are reported by Bergamaschi et al. (2013) and Houweling et al. (2014). Comparisons with inversions based on early retrievals from GOSAT (Monteil et al., 2013; Alexe et al., 2015) show good agreement with bias-adjusted values from SCIAMACHY, the GOSAT data being more precise and less biased, but sparser. An estimate for the emission of methane from fracking has been made using SCIAMACHY data and information on boundary-layer winds from reanalysis (Schneising et al., 2014).

Surface flux estimates for both carbon dioxide and methane are currently updated on a routine basis and made available as a Copernicus service at atmosphere.copernicus.eu. More generally, significant progress is expected over the roadmap period as new instruments are deployed in space, as experience is gained and as inversion systems, including their transport models, are improved. The case for a complementary expansion of in situ observation is advanced by Ciais et al. (2014).

Inversion may also be used to estimate the emission and deposition of a range of other atmospheric constituents. Examples are the estimates of:

- varying global carbon monoxide emissions over a ten-year period using a chemical transport model with constraining observations from the MOPITT instrument on EOS/Terra (FortemsCheiney et al., 2011);

- emissions and the chemical state for an 18-day ozone-pollution episode using a regional airquality model with constraining in situ surface observations of nitrogen oxides (NOx), sulphur dioxide and ozone (Elbern et al., 2007);

- emissions of NOx at $25 \mathrm{~km}$ resolution using a Kalman filter approach and a regional air-quality model with constraining satellite data retrievals from GOME-2 and OMI measurements (Mijling and van $\operatorname{der}$ A, 2012);

- the global production of NOx by lightning, using a chemical transport model with constraining data from multiple (MLS, MOPITT, OMI, TES) satellite instruments (Miyazaki et al., 2014); 
- emissions of $\mathrm{SO}_{2}$ and several primary aerosol species, using a global model of intermediate complexity with constraining total and fine-mode aerosol optical depth data from MODIS (Huneeus et al., 2013);

- heights and rates of volcanic ash injection using Lagrangian particle dispersion models with constraining data from the SEVIRI instrument on Meteosat (Kristiansen et al., 2012);

- the caesium-137 source for an accidental release from a nuclear power plant, using a mesoscale transport model with constraining atmospheric concentration and deposition observations (Winiarek et al., 2014).

\section{Use of observations in integrated Earth-system modelling and its applications}

The use of observations, either directly or through processed datasets, is discussed in the context of applying and improving Earth-system models. Topics covered are monitoring and interpretation, including the attribution of effects to causes, the evaluation of models, and prediction and projection.

\subsection{Monitoring and interpretation}

Monitoring the changing state of the Earth system takes place on spatial scales that vary from the local to the global. Impacts on lives are experienced locally and local environmental parameters such as those related to heat stress and air quality have to be checked and exceedances acted upon. Regional monitoring is needed for transboundary transport of air pollution and river flow. Globally, assessment of the net capacity for food production and the setting of bounding targets for greenhouse-gas concentrations or temperature rise bring with them needs to watch the approach of the system towards critical limits. The monitoring itself may involve data that relate to a single variable or group of closely related variables, but an integrated approach is often needed to understand the reasons behind the variability and trends of particular variables, whether local, regional or global, and to formulate effective programmes of mitigation and adaptation, which in turn may have local, regional and global elements.

It is beyond the scope of this study to discuss the many uses of observations for monitoring individual components of the Earth system, although several are referred to in the variable-byvariable discussion of Earth-system observation given in section 3. A key need is for homogenization of the time series of data from individual types of in situ observation, whether it be to take into account changes in bias in measurements from instruments such as radiosondes or changes in the siting or exposure of observing stations making near-surface measurements. Likewise, account has to be taken of drifts or inter-platform changes in measurement bias from satellite-borne instruments. These are requirements that apply whether the observations are used directly, built into a single-variable data product or combined with other types of observation in a more comprehensive reanalysis. Allowance also has to be made for inadequacies and changes in spatial and temporal sampling. This can be due to variations in completeness of in situ observational coverage, a source of uncertainty in estimates of global-mean surface temperature, for example. It can also be an issue in satellite data products, for example when data relate only to cloud-free conditions or sampling of the diurnal cycle changes due to orbital drift. Sampling is a specific issue for monitoring changes in the frequency or intensity of rare but severe events, for which the length 
of the period of good observational record may be a fundamental limitation, affecting also the use of observations for assessing the performance of models in this respect. Sampling is more generally an issue for monitoring intermittent events such as rainfall.

A role for reanalysis in monitoring has been alluded to in section 5.3 , and the particular contribution it can make to observational bias estimation and adjustment has been noted in section 5.5. A number of caveats have nevertheless to be heeded. It is important, for instance, to assess the reliability of the way reanalysis fills in gaps where datasets based on direct gridding of observations lack coverage. Critical in this regard are the quality of the assimilating model and the extent to which the infilling is constrained by observations of related variables, for example by observations of seaice cover in the case of high-latitude marine surface air temperature, which depends sensitively in winter on whether the underlying surface is ice-covered or open sea. In addition, bias in the assimilating model becomes an issue for monitoring change where observational coverage changes over time, as changes in coverage change the extent to which model bias is countered by assimilated observational information.

Generally important in this context are the use where possible of independent observations to evaluate data products, and the comparison of the results from different producers and from different methods of production. Consistency of results for related sets of variables can provide a further source of confidence. This may involve, for example, comparing anomaly patterns and strengths at different levels in the atmosphere where different types of observation provide the principal sources of information. It may alternatively involve evaluating the consistency of a set of estimates, of which a prime example is provided by the recent balancing of the observationallybased budget of mean sea-level rise in terms of the contributions from thermal expansion of the oceans, melting of glaciers and ice sheets, and changes in water storage on land. This was identified by Church et al. (2013) as a significant advance from AR4 to AR5 in physical understanding. Closure of the overall water-cycle budget of the atmosphere using observational products remains a challenge nevertheless.

The ability to forecast future events or simulate past ones using comprehensive models enables controlled modelling to be used to explore factors that may or may not be important for the events. This includes, for example, determining the importance of particular observations for enabling successful weather predictions (WMO, 2012), or the importance of particular forcing factors for accurately modelling temperature change over the past century or more (IPCC, 2013). Constraining a model with wind and temperature information from reanalysis has been used to reconcile halogeninduced loss of stratospheric ozone with the observed record of total-column ozone (Shepherd et al., 2014) and to understand variations over time in stratospheric water vapour (Hegglin et al., 2014). A number of fields are prescribed in models, which may include surface roughness, soil type, land cover, aerosol and trace-gas concentrations or emissions, depending on the complexity of the model. Models accordingly benefit from better data on these fields, and such data may be assessed through their impact on the performance of the models that employ them, in reanalysis, forecasting and simulation.

Attribution of events to causes has a high profile in the context of possible impacts of climate change. It is needed for effective working of the international mechanism for loss and damage established by the Parties to the UNFCCC, which recognizes the need to enhance knowledge and 
understanding, including through collection, sharing, management and use of data, and is concerned with both extreme weather events and slow-onset events associated with adverse effects of climate change. More generally, there is a requirement from commercial and governmental sectors, and from the general public, for assessments of the extent to which weather-related risks can be discerned as having changed due to human influences on climate or due to changing vulnerabilities to natural variations in climate. Attribution may also be important in other challenging types of situation, for example air-pollution events that involve both cross-boundary transport of pollutants and local emissions, or cases of low or high cross-boundary river flow.

\subsection{Model evaluation}

\subsubsection{Basic aspects}

Systematic evaluation of models through comparisons with observations is a prerequisite for the models to be used with confidence and needed for identifying what has to be improved. When a new version of a model becomes available, it too has to be evaluated thoroughly to ensure it performs as expected. No single evaluation technique or performance measure is adequate for determining the fitness of models for the range of purposes for which they are applied. Instead, a variety of techniques and measures must be used to provide a comprehensive overview of model performance. Common metrics have to be adopted to ensure that the models developed by different institutions can be compared. A move towards adopting metrology standards for comparing models with a reference is envisaged (e.g., Widlowski et. al., 2013).

Assessment of the forecasts and simulations provided by models may utilize either direct comparisons with observations or comparisons with processed observationally-based datasets. This brings additional requirements, including for accessibility of datasets and assessments of their quality. The Obs4MIPs and Ana4MIPs initiatives (www.earthsystemcog.org) are being developed to facilitate the use of data products specifically to support the evaluation and intercomparison of climate models, complementing the general availability of model results through the CMIP and other intercomparison projects. General information and guidance on observationally-based datasets are available from websites such as climatedataguide.ucar.edu and reanalyses.org. Data portals include NOAA's www.gosic.org and GEO's www.geoportal.org, while CEOS and CGMS provide dataset information at ecv-inventory.com. The operational Copernicus services are also expected to play a substantial role in this regard. These activities have mostly been established quite recently, and refinement and rationalization are likely over the coming ten years.

The evaluation of models using past observations is most reliable for those variables and phenomena for which direct observations exist, although reanalysis may be useful for other variables if they are constrained indirectly by assimilated observations of related quantities. Nevertheless, in many cases the lack or insufficient quality of long-term observations, whether of a specific variable, an important process or a particular region, remains an impediment.

\subsubsection{Regime and process separation}

A number of techniques have been developed to isolate particular regimes or processes within Earth-system models for the purpose of assessment. One involves the so-called "regime-oriented" approach for evaluating processes. Instead of the more typical averaging of model results in time or space and comparison with corresponding averages of observationally based products, results may 
be averaged within categories that describe distinct dynamical or physical regimes of the system, and performance accordingly assessed regime by regime. This enables regimes to be identified where more or less reliability can be placed in a forecast or a climate projection, and helps in the setting of priorities for model development. In the case of the atmosphere, for example, it may involve compositing different instances of a particular type of synoptic event, such as mid-latitude blocking or African easterly waves, but may more simply involve focussing on selected time averages for persistent local or regional phenomena such as the stratus cloud decks over the western coasts of continental areas, which are often associated with warm biases in coupled ocean models. Process-based metrics for the ENSO are another example; these are being developed as a communal activity organised within CLIVAR (www.clivar.org/research-foci/enso).

Another approach supporting model development involves isolating model components or parameterizations in off-line simulations, such as from a single-column version of the atmospheric component. Results of such simulations may be compared with measurements from dedicated field studies or with results from more detailed models specific to the processes being considered. Use may be made, for example, of spectral radiation measurements at the top of the atmosphere and the surface to test, refine and verify columnar physics effects in global models such as related to water vapour, lapse rate and cloud feedbacks. Boundary-layer or cloud processes may be studied by comparing with results from so-called "large eddy simulation" or "cloud resolving" models respectively. Collaborative international programmes such as the Radiative Transfer Model Intercomparison Project (Collins et al., 2006), the Radiation Transfer Model Intercomparison) for the Intercomparison of land-surface Parameterization Schemes (Widlowski, et. al., 2011) and the Cloud Feedback Model Intercomparison Project (CFMIP; cfmip.metoffice.com) often provide an important organizational framework for such studies.

\subsubsection{Instrument simulators}

Satellites provide important data for model evaluation. The conventional approach has been to convert the measured radiance data into data on "model-equivalent" geophysical variables, the process known as retrieval. Limitations in what can actually be sensed from space poses a challenge, however, as various assumptions have to be made in order to carry out the retrieval. An alternative approach is to derive "observation-equivalent" data from models using radiative transfer calculations to simulate what the satellite would provide if it were sensing the model rather than the real state. This approach is usually referred to as an "instrument simulator" when used to evaluate a climate model. An observation simulator package is, for example, provided under CFMIP.

The same approach of mapping model fields to what is observed from space is well established in data assimilation, where model fields are adjusted so that the implied radiances match what the satellites measure to a degree consistent with other observational and model constraints. In this case the simulator is usually referred to as a fast forward radiative transfer model; the adjoint of the forward model is also required in variational assimilation to derive the adjustment needed to the model fields. The extent to which a good match to radiance and other observational data is already provided by the background forecast, is achieved by the analysis and is maintained into subsequent longer forecasts provides valuable diagnostic information, although interpretation of results may require care. Benefits for climate modelling should ensue from a more widespread use of initialvalue techniques in their evaluation, discussed further in section 6.2 .6 below. 


\subsubsection{Evaluation at component and integrated levels}

Individual model components (the atmosphere, the ocean and so on) are first typically evaluated in isolation as part of the model development process. For instance, the atmospheric component can be evaluated by prescribing sea-surface conditions or the ocean and land components by prescribing atmospheric conditions. Subsequently, the various components are assembled into a comprehensive model, which then undergoes a systematic evaluation. At this stage, a small subset of model parameters typically remains to be adjusted so that the model adheres to large-scale observational constraints. As this final parameter adjustment procedure, usually referred to as tuning, aims to match the observed behaviour of the climate or Earth system, it entails judgment as to what constitutes a skilful representation of the system. For instance, maintaining the top-of-theatmosphere energy balance in a simulation of pre-industrial climate is essential to prevent the climate system from drifting to an unrealistic state. Current models almost universally contain adjustments to parameters in their treatment of clouds to fulfil this important constraint.

Model tuning directly influences the evaluation of climate models, as the quantities that are tuned should not be used in model evaluation. Quantities closely related to those tuned provide only weak tests of model performance. The use of data is integral to the model development process, and this complicates the construction of critical tests. Nonetheless, by focusing on those quantities not generally involved in model tuning while discounting metrics clearly related to tuned features, it is possible to gain insight into model skill. The concurrent use of many model quantities, evaluation techniques, and performance metrics that together cover a wide range of emergent (or un-tuned) model behaviour, ensures a stringent test of model quality.

\subsubsection{Metrics}

The application of common metrics based on standards enables the performance of different forecasting systems or climate models to be compared. Coordinated activity dates back to the early 1980s in the case of numerical weather prediction, initially under a research programme (Lange and Hellsten, 1984), and continues today on an operational basis under the auspices of the WMO Commission for Basic Systems. The current operational system entails a monthly exchange of various agreed metrics of forecast performance computed by each forecasting centre. Forecasting centres also exchange a subset of their forecast fields, enabling an individual centre to compute its own metrics to compare its forecasts with those of others. For example, Simmons and Hollingsworth (2002) used such metrics in their documentation and interpretation of some twenty years of improvement in forecasts from global systems. Both observational data and the NWP analyses derived from them are used in calculating metrics; the distinction becomes important for regions more poorly constrained by observations or where model performance is relatively poor. In this case cross-validating the forecasts of one centre against the analyses of another can be instructive. Metrics may be chosen by institutions to set strategic targets or for year-on-year tracking of progress. Examples are the headline scores published at www.ecmwf.int and the UK and Global NWP Indices reported at www.metoffice.gov.uk. Haiden et al. (2014) provide an example of how ECMWF reports annually on metrics of forecast performance and system upgrades.

A corresponding effort for climate models has been much longer coming. Reasons have been discussed by Gleckler et al. (2008), who proposed a set of metrics for atmospheric variables and demonstrated a number of ways of presenting results for a large group of models. The AR5 WG I 
chapter on the evaluation of climate models (Flato et al., 2013) presents numerous more-recent examples of metric-based performance comparisons. The chapter also serves to indicate the observationally-based datasets available for use in such evaluations. Eyring et al. (2015) report the development and illustrate the application of a communal software tool that facilitates evaluation of a set of Earth-system models using a common set of performance metrics and diagnostics.

A point made by Gleckler et al. (2008) was that despite the increasing use of metrics it was not generally possible to identify a "best" model from them, noting that this would almost certainly depend on the intended application. The same conclusion may be drawn from the set of evaluations presented in AR5. For example, two models from the Hadley Centre score among the best overall according to a set of relative error measures of CMIP5 model performance for the atmosphere, based on a global seasonal-cycle climatology for 1980-2005 (Figure 9.7 of Flato et al., 2013), but are the poorest in reproducing the observed increase in ocean heat content over the same period (Figure 9.17, ibid.).

There has been difficulty in establishing metrics that quantify the strength of the interactions in coupled systems. This was noted in particular by WCRP's Global Energy and Water Exchanges project in establishing its imperatives for 2013 and beyond (www.gewex.org). Its Global Land Atmospheric Coupling Experiment, for example, was successful in identifying how strongly soil moisture was coherent with precipitation at the model resolution scale of order $100 \mathrm{~km}$, but other than a relative ranking of strength was unable to provide quantitative metrics or understanding of governing processes or their accuracies.

\subsubsection{Initial-value techniques}

Knowledge of the present state of the atmosphere is essential for forecasting the weather for a few days ahead. In contrast, climate predictions and projections simulate the statistics of weather seasons to centuries in advance. Despite their differences, both weather predictions and climate projections are generally performed with very similar atmospheric model components, and global weather-prediction systems increasingly include other components more typical of climate models, as discussed in section 4.1. The atmospheric component of a climate model, and other components as appropriate, can be integrated as a weather prediction model if suitably initialized. This allows parameterized sub-grid scale processes to be tested without the complication of feedbacks that can substantially alter the underlying state of the atmosphere when models are integrated over extended periods of time.

Understanding the source of systematic errors in climate models is challenging because of such coupled feedbacks and compensating errors. The developing seamless approach is based on the premise that the identification and correction of short-term climate-model errors have the potential to improve the modelled climate on longer time scales. Initialized atmospheric simulations of a few days have been used to compare model treatments of fast physical processes, notably involving convection and clouds. This is now being extended to the use of initialized seasonal to decadal hindcasts to relate transient weekly-to-monthly errors of the oceanic and atmospheric components to pervasive long-term errors of coupled models. Despite substantial developmental efforts, the latest generation of coupled models shows only modest improvement. Using initial-drift analysis helps to move from the current rather ad hoc approach towards a systematic, bias-targeted, prioritysetting approach to model development. Vannière et al. (2013) provide an example, using seasonal 
hindcasts to seek the root cause of error in representing the equatorial Pacific cold tongue in coupled climate models.

\subsection{Prediction and projection}

\subsubsection{General roles and requirements for observations}

Observations play multiple roles in the prediction of forthcoming changes in the Earth system and the scenario-based projection of possible future courses of the system. The importance of different types of observation and of where they are made generally changes with the range of the prediction or projection. As particular observations may serve multiple applications involving different time ranges, priorities for observations in general vary from application to application, as well as from region to region.

Observations are needed to provide the initial conditions and verifying data for predictions, both close to real time for operational use and from past dates to provide starting points for the hindcasts (or reforecasts) that are used to test system changes, to provide users with indications of the quality of current operational systems and to implement schemes for calibrating forecasts to offset model limitations. In the latter case use may be made of routine reanalyses, or observations may be reprocessed especially for the specific purpose required. Observations and socio-economic data related to external forcings or emissions of key trace species are also needed. As discussed further below, these are required in particular for longer-range forecasting and to guide the setting of scenarios for projections.

Basic questions as to which variables need to be observed, where they need to be observed and with what spatial and temporal resolution are addressed by programmes such as GCOS in the case of observations serving climate needs. It is not the function of this report to produce or reproduce extensive and comprehensive specifications; indicative requirements and guidance covering much that is relevant to this report can be found in the WMO OSCAR database.

Timeliness of delivery of observational data is another factor to be taken into account. Delivery needs to be as prompt as reasonably possible, within from about one to a few hours of the measurement time in the case of short- and medium-range weather forecasting. User desire for information as early as possible has, however, to be balanced against the benefits of waiting for the arrival of late or better-processed data. For seasonal forecasting this can mean a delay of around 1015 days to receive satellite altimeter data on sea-level with better orbit specification or biascorrected Argo salinity data, for example. Delays can be longer for specialized data for verification, and recovered or previously restricted observations can be valuable many decades after they were made when used in reanalysis or other forms of processing for study of climate change.

Nevertheless, given the increasing variety of data used in operational weather forecasting systems and the practice of extending reanalyses in "climate data assimilation mode" a few days at most behind real time, it can be foreseen that increased attention will need to be paid to planning nearreal-time data supply streams from future satellite missions.

Operational prediction requires that the observing system that supports it be resilient to failure. This concerns both key satellite observing systems and the critical elements of the ground-based telecommunication systems that transmit both in situ and space-based data. This implies some 
degree of redundancy. Temporal stability of observations, or at least of reanalyses or other data products derived from them, are requirements not only for monitoring a changing environment but also for deriving calibrations of forecasting-system outputs (Kumar et al., 2012). Other requirements include provision of metadata on observational uncertainty, for direct use in the selection of observations to assimilate and the assignment of observation error statistics in the data analysis, and for choosing appropriate perturbations to apply to the observations when used in ensemble data assimilation systems.

\subsubsection{Predictability and uncertainty}

Predictability can refer to either the current or the fundamental limit to which some property of a variable may be predicted. As discussed by Thompson (1957) for weather prediction, it may be quantified as the length of the time range over which the error (or uncertainty) of a representative sample of forecasts lies significantly below the error (or uncertainty) of a corresponding sample of guesses such as provided by predicting climatology or persistence of the initial state. Thompson estimated an inherent error doubling time of two days based on instability theory for mid-latitude wintertime flow, and a corresponding predictability limit of about a week based on the observational network of the time, noting both the potential to extend this by refining the network and the diminishing returns to be expected from continuing refinements. Lorenz (1995), in an article entitled "Predictability - a problem partly solved", noted how the doubling times of small differences in global-model states reduced from about five days for the early atmospheric models of the $1960 \mathrm{~s}$ to around 1.5 days for the ECMWF global forecast model in the mid-1990s. Since then, values have settled at 1.2-1.4 days for ECMWF forecasts. The same period has seen the establishment of ensemble prediction, the running of multiple forecasts from perturbed initial conditions, perhaps also with a stochastic element to the model formulation, as a way of providing dynamic estimates of forecast uncertainty and potential weather scenarios. However, even for the probabilistic forecasts that such systems produce, there remains a predictability limit beyond which information other than climatologically informed guesswork cannot be supplied on the instantaneous state of the weather.

The error of instantaneous forecasts does not saturate completely at a particular range, however. Although continued growth of systematic model error can contribute, there is an element of atmospheric predictability that is associated with longer-term variations in the atmosphere's oceanic and terrestrial lower boundary conditions, in its chemical composition and in the solar radiative input it receives. As has long been appreciated, this "climatic predictability" (Lorenz, 1975) manifests itself in skilful prediction of temporal and spatial means and other statistics, with averaging typically taken over longer periods and larger areas for longer forecast ranges.

Uncertainty in climate-change projections is discussed in the contribution of Kirtman et al. (2013) to AR5, based an analysis of CMIP5 results that builds on an earlier CMIP3 analysis by Hawkins and Sutton (2009). Uncertainty is partitioned into contributions from three sources. These are the natural internal variability intrinsic to the climate system, imperfect knowledge of future changes in natural and anthropogenic forcings, and limitations of the models needed to estimate the responses to changed forcings. Uncertainty over future anthropogenic forcings was represented in CMIP5 by scenarios for concentrations of species that affect radiative forcing. There is also model-related uncertainty in the link between such atmospheric concentrations and the underlying anthropogenic emissions of the species themselves or their precursors. 
(a)

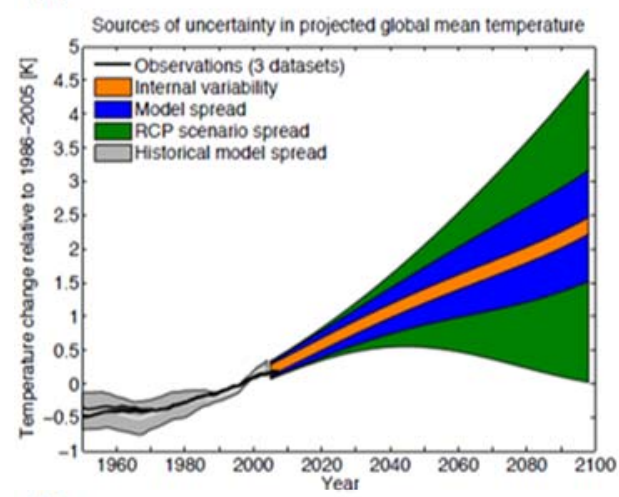

(c)

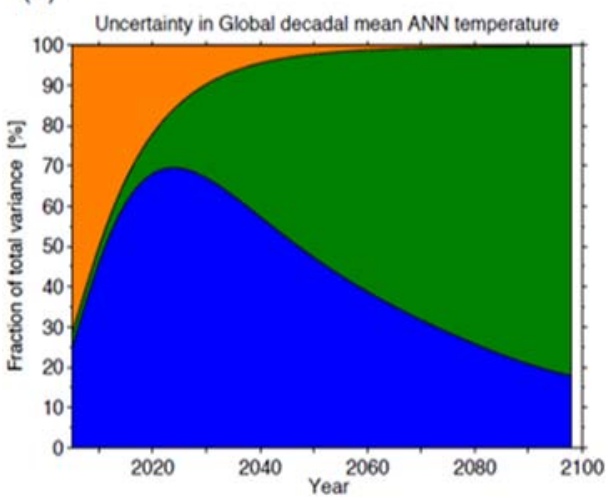

(e)



(b)

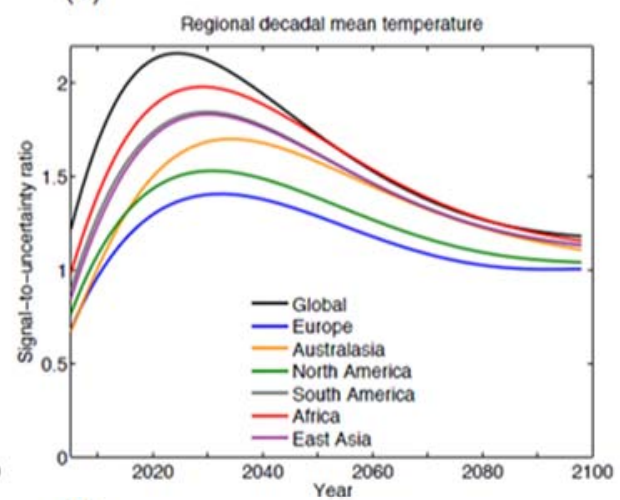

(d)

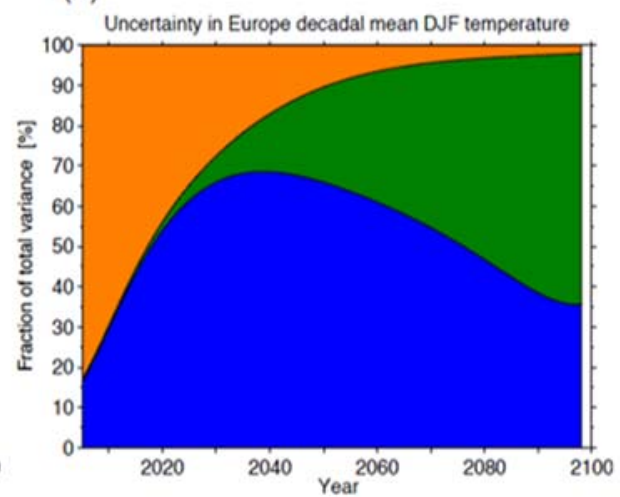

(f)

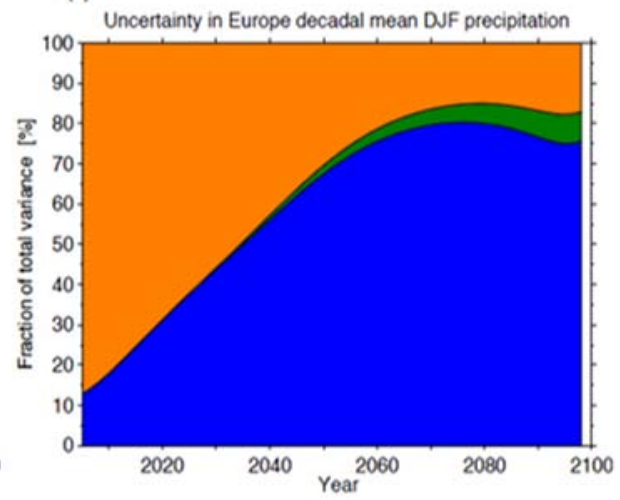

Figure 8 Sources of uncertainty in climate projections as a function of lead time based on an analysis of CMIP5 results. (a) Projections of global mean decadal mean surface air temperature to 2100 together with a quantification of the uncertainty arising from internal variability (orange), model spread (blue) and $R C P$ scenario spread (green). (b) Signal-to-uncertainty ratio for various global and regional averages. The signal is defined as the simulated multi-model mean change in surface air temperature relative to the simulated mean surface air temperature in the period 1986-2005, and the uncertainty is defined as the total uncertainty. (c-f) The fraction of variance explained by each source of uncertainty for: global mean decadal and annual mean temperature (c), European $\left(30^{\circ} \mathrm{N}\right.$ to $75^{\circ} \mathrm{N}, 10^{\circ} \mathrm{W}$ to $40^{\circ} \mathrm{E}$ ) decadal mean boreal winter (December to February) temperature (d) and precipitation (f), and East Asian $\left(5^{\circ} \mathrm{N}\right.$ to $45^{\circ} \mathrm{N}, 67.5^{\circ} \mathrm{E}$ to $\left.130^{\circ} \mathrm{E}\right)$ decadal mean boreal summer (June to August) precipitation (e). Source: Kirtman et al. (2013), after Hawkins and Sutton (2009), where further detail may be found.

Caveats notwithstanding, the broad conclusions from results such as illustrated in Figure 8, taken from Kirtman et al. (2013), are clear. Internal variability dominates uncertainty for the first decade or more ahead, depending on region, season and variable. Thereafter, modelling becomes the largest source of uncertainty. Uncertainty in forcing, estimated here from the spread of projections for different CMIP5 "Representative Concentration Pathway (RCP)" scenarios, comes into play as the 
time range extends to multiple decades. Its extent evidently varies substantially depending on which aspect of climate is being considered.

\subsubsection{Prediction from days to decades}

There are needs for forecasts of how the Earth-system will change over multiple time ranges. The move to view and implement prediction in a more seamless way across the time ranges has been discussed already, but different considerations nevertheless come into play when considering predictions with different lead times. These are associated with aspects such as the fundamental limits to predictability, the relative importance of observations of various types and the lengths of observational records.

\subsubsection{Short to medium-range prediction}

Needs for information on what will happen within the Earth system are generally met in most detail or with least uncertainty, and with substantial demonstrable socio-economic benefit, in shortand medium-range weather forecasts out to days or at most around two weeks in advance. Such forecasts are relatively well served by the observations that are critically needed to define initial conditions and benefit from use of models that are operating over a time range for which deficiencies in formulation generally do not substantially degrade the realism of the fields they produce. Improvement of forecasting systems and assessment of the importance of the various types of atmospheric and surface observations benefit from an extensive set of past conditions on which to test system changes and data impacts, from the relative ease and rapidity with which forecasts can be verified, and from extensive user feedback.

As both the synoptic-scale accuracy of forecasts and the resolution and other aspects of model realism have increased, so the use of and demand for more accurate direct forecasts of weather elements have increased. This in turn requires good initial specification of land-surface variables such as soil moisture, vegetation and snow cover, in addition to the atmospheric fields of primary importance for the synoptic quality of forecasts. The importance of data on ocean-wave and sea-ice conditions, and the ongoing evolution of global forecast models to include coupled ocean circulation and sea-ice components, and coupled schemes for atmospheric chemistry, accompanied by coupled data assimilation, have already been discussed. These support marine, land-surface and air-quality forecasts in their own rights as well as adding to the skill of weather forecasts.

\subsubsection{Sub-seasonal to seasonal prediction}

Sub-seasonal prediction beyond the time range of medium-range weather forecasting received less attention for some time than prediction for a season or more ahead. Early experience with monthly forecasting was one of limited benefit, while clearer prospects were seen for longer-term prediction due to the predictability of tropical SST and linked conditions in the tropical atmosphere, and the associated influence on extratropical weather-regime statistics on the longer, seasonal timescale (Palmer and Anderson, 1994).

Several factors have led to renewed interest in prediction for the sub-seasonal to seasonal time range. One is better understanding and prediction of the Madden-Julian Oscillation, a major component of tropical intraseasonal variability with impact also at middle and high latitudes. Developments in coupled atmosphere-ocean modelling and data assimilation are improving the prediction of sub-seasonal SST variations and their influences on the atmosphere. Anomalous soil- 
moisture, snow and sea-ice conditions are other sources of predictability for the atmosphere beyond the two-week time range for certain regions and times of year. In addition, substantial changes in the stratospheric winter polar vortex have been found to be followed by extended periods of anomalous tropospheric flow. The relatively slow nature of meridional and vertical transport in the stratosphere may also enhance predictability through the persistence of anomalous radiative forcing associated with anomalies in the distributions of trace species such as water vapour and ozone.

This renewed interest has led to establishment of a sub-seasonal to seasonal prediction project jointly by the WCRP and WMO's World Weather Research Programme. The research implementation plan for this project (WMO, 2013) provides more on its scientific rationale and programmatic linkages. The plan does not address specific additional observational needs. However, advances for this range of prediction over the coming ten years can be expected to place increased emphasis on data relating to snow properties, soil moisture, near-surface ocean conditions and seaice state, on observation in general for the tropics, upper stratosphere and mesosphere, and on associated capabilities for data assimilation and reanalysis.

\subsubsection{Seasonal to annual prediction}

Of special importance for seasonal prediction are the variations of tropical SST in the Pacific sector associated with ENSO. The associated changes in convective heat sources lead to widespread changes in atmospheric circulation and a net warming of the atmosphere for a period of a year or so. Socio-economic impacts are substantial. The central role ENSO plays for seasonal forecasting is enhanced by its relatively high potential predictability, which is inherently dependent on oceanic initial conditions, and in particular on the precursor provided by equatorial heat recharge. Realising the potential depends critically on the adequacy of initial conditions for the oceanic component of the coupled models used for prediction. Internal equatorial wave dynamics play a role, but it is now accepted that linear dynamics is insufficient for predicting the evolution of SST: not every eastward propagating Kelvin wave leads to an SST anomaly of the expected sign, and intensity varies from one event to another. Indeed, the observed diversity of ENSO is the result of differences in wave/meanstate interaction, the role of the equatorial and extra-equatorial Pacific Ocean and other tropical ocean basins in modulating large scale atmospheric convection, and the response of the ocean to aspects of Westerly Wind Events such as intensity, timing and fetch (e.g Menkes et al., 2014), without excluding the effects of salinity on vertical mixing and horizontal pressure gradients (Zhu et al., 2014). Observational needs are being reviewed in the light of changing observing and forecasting capabilities, in a Tropical Pacific Observing System project (TPOS 2020; tpos2020.org/) that will also consider biogeochemical aspects and human influences.

The skill of seasonal forecasting systems has increased since they became operational. The improvement has been attributed in similar degree to better observationally-based initialization of the ocean and improved coupled forecast models (Figure 9; see also Stockdale et al. (2011)). Improved initialization reflects not only the contribution of the ocean observing system, but also improved atmospheric surfaces fluxes (due partly, in turn, to improved atmospheric observation) and better exploitation of the available observations by more advanced data assimilation methods and assimilating models.

Determining observing-system impacts and implications for observing-system design is more difficult for seasonal prediction than for weather forecasting, due to sampling limitations that arise 
from a shorter comprehensive observational record for the ocean and the longer time ranges over which impacts are felt. The initialization problem is even more daunting for biogeochemical and ecosystem prediction, including as it does critical linkages to components of the physical climate. Assessing the reliability of forecasts is also hampered by limitations of sample size.

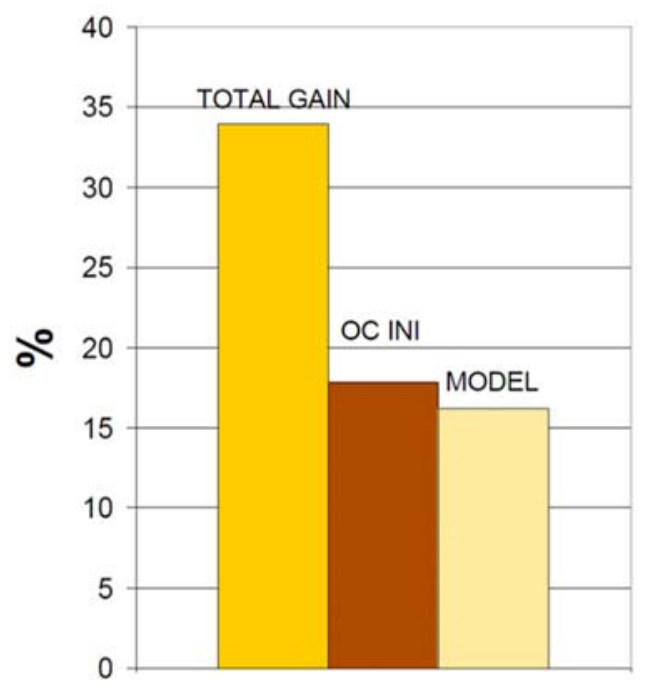

Figure 9 Progress in the seasonal forecast skill of the ECMWF system since it became operational around 1996. The left-hand bar shows the relative reduction in mean absolute error of forecasts of sea-surface temperature in the eastern Pacific (NINO3 area) integrated over 1-6 month forecast lead times. Contributions from improved ocean initialization (middle bar) and model development (right-hand bar) are almost equally important. Developments in ocean and atmosphere models also contribute to the improvement in ocean initialization. Source: Balmaseda et al. (2010).

\subsubsection{Decadal prediction}

Uncertainty exists as to how climate will change from a year to a decade or so ahead, in particular at regional and local scales for which natural variability is large. Figure 8 indicates that this uncertainty has two main sources: the internal variability of the climate system and the differences between the responses of models to changing external forcing, including hydrological, cryospheric and biogeochemical feedbacks. However, the figure and discussion given in section 6.3.2 relate to ten-year means and are based on the particular setup of the CMIP5 projections. They thus do not represent fully the influences of changes in volcanic activity and solar radiative inputs, which are discussed separately in sections 6.3 .5 and 6.3 .6 below.

Aside from such effects, the key questions for decadal prediction concern the extent to which the uncertainty due to internal variability out to a decade or so ahead can be reduced by observationally-based initialization, the extent to which the forced change contributes to the skill of prediction at this time range when scenario uncertainty generally remains small, and the extent to which model improvement will contribute to improved predictions.

The status of decadal prediction has been assessed by Kirtman et al. (2013) in AR5 and reviewed subsequently by Meehl et al. (2014). Many results show, as expected, that predictability associated with the initial state tends to decay with time. The range over which initialization adds value depends on the region and the variable considered. Significant skill from initialising hindcasts is found to last for up to around a decade in trends for the Atlantic basin, especially in the subpolar gyre, while skill is much more rapidly lost in the Pacific and Indian Oceans. There is limited evidence 
suggesting that some climatic events such as the Sahel drought of the 1970s and 80s, the mid-1970s climate shift in the Pacific, the increase in European winter temperature from the 1970s to the 1990s and variations in Atlantic hurricane activity may be predictable, but it is also found that the predictability of internally generated decadal changes is generally low over land in surface temperature and marginal in precipitation, limited to very few areas.

The observational needs for decadal prediction are understood to be similar to those for seasonal to annual prediction, but with a greater need for information on the deep ocean. Synthetic observing system experiments suggest that observations below $2000 \mathrm{~m}$ should play a role, especially in the prediction of the Atlantic Meridional Overturning Circulation. Initialising large scale modes of variability such as the Pacific Decadal Oscillation may also be important. Sea-ice, land and snow cover all have the potential to provide multi-year predictability. The lack of an observational record long enough to explore and develop decadal prediction to the full is a fundamental one that will be alleviated only a little by the decade of new observations and improved reanalyses that will become available over the period of this roadmap. Nevertheless, with underlying predictive signals from continued anthropogenic emissions and solar variability, and with continued model development and concerted international collaboration a better appreciation of the prospects for decadal prediction should emerge over the period. Decadal prediction may then become a component of operational climate services, providing general information on the likely path of global or large-scale climate change, even if skill levels do not allow uptake of products for a wide range of user applications.

\subsubsection{Projection of climate change}

The term projection is widely applied in the context of climate change to refer to model integrations for estimating the future conditions that would occur given prescribed scenarios for uncertain factors causing climate change, notably future anthropogenic emissions of key gases and aerosols, and future land use. Projections may also be run to assess possible benefits and side effects of proposed geoengineering approaches to mitigating climate change. The alternative terms non-initialized predictions and non-initialized simulations are also used, for example in AR5, but this is a misnomer as projections usually entail initialization of some components of the climate or Earth system. For example, initial greenhouse-gas concentrations were set to current observed values in defining the RCPs used in the long CMIP5 integrations assessed in AR5. Run as continuations of historical simulations for the period since 1850, the CMIP5 projections are initialized through observationally based elements of the specified historic forcing and boundary fields. Together with the use made of observations in designing and refining models so as to reproduce past climate, this results in starting conditions for projections that are constrained not to differ significantly from the present observed large-scale state of the climate system. Projections may accordingly be refined by better availability and use of past and present observations, even if their primary sensitivity is to differences in the set of scenarios that they encompass. Initialising observational data that could benefit projections for the scenario-sensitive climate time range include those of deep-ocean conditions and slowly varying cryospheric and other land conditions.

Moreover, scenario-based projections have a role to play even on short time scales when the values of weather variables need to be strongly constrained by observations, in either a reanalysis or a forecasting context. For example, development of environmental policy for control of emissions 
affecting air quality may make use of scenarios that explore the impacts of proposed limits to emissions. Implementation of policy may benefit from running emission-reduction scenarios in nearreal-time to decide whether to impose emission restrictions on an emergency basis when severe pollution episodes are predicted. Scenarios for volcanic emissions could be run when a particular volcano is observed to be in a potentially imminent eruptive state or in the early stages of an actual eruption when the amount and height of ash and gas injected into the atmosphere are not well known.

The setting of scenarios in general will be based in part on observational and other forms of data relating to anthropogenic emissions, land-cover changes and any other uncertain forcings that are taken into account. As the capabilities of integrated modelling expand, eventually to include modelling of human systems, scenarios should become more directly based on the actual decisions to be made with regard to emissions, land use and other environmental matters, rather than separately determined specifications of quantities such as the possible future concentration pathways for greenhouse gases.

\subsubsection{Volcanic influences}

Volcanic eruptions are generally categorized as providing a variable and largely unpredictable external forcing of the climate system (section 2.3), although they might equally be regarded as a largely unpredictable component of the internal variability of the full Earth system. Either way, they are a factor behind uncertainty in climate prediction and projection. IPCC (2013) qualifies its "medium confidence" prediction of a global-mean surface temperature change for the period 20162035 relative to $1986-2005$ in the likely range of $0.3^{\circ} \mathrm{C}$ to $0.7^{\circ} \mathrm{C}$ by noting that it is based on the assumption that there will be no major volcanic eruption in the period. It discusses how eruptions similar in effect to those of Agung, El Chichón and Pinatubo that occurred within a thirty-year period in the $20^{\text {th }}$ century would each reduce near-surface temperatures for a year or two by an amount in the lower half of the otherwise predicted range of global temperature increase, with weaker continuing effects possible due to the longer response-time of the ocean. The occasional occurrence of more extreme eruptions in earlier centuries has also to be kept in mind.

Nevertheless, volcanic eruptions can imbue the system with predictability. This occurs for predictions that start soon after eruptions. The forecasting problem then becomes one internal to the climate system, as it is one of predicting the response to anomalous levels of volcanic aerosols already in the atmosphere, and thus depends on the observation of these aerosols, assimilation of the resulting data to construct initial conditions, and modelling of the subsequent aerosol content and its radiative effects on temperature. Challenging as this is in practice, it offers the prospect of an additional component of skill in predictions for time ranges out to the multi-annual.

Challenges for short- and medium-range forecasting are related, but different. Here there is an emphasis on forecasting the immediate spread of the ash and gases injected by volcanoes, because of the directly harmful effects they can have. Again, observations to determine initial conditions are critical. Also important can be the effect of volcanic aerosols on infrared sounding measurements made from space. Data from sounding channels that provide information on humidity can also be sensitive to unusual amounts of aerosol, and their use in data assimilation may be problematic if proper account is not taken of the aerosol effects. This was, for example, one of the lessons learnt from undertaking the ERA-40 reanalysis (Uppala et al., 2005). 


\subsubsection{Solar irradiance}

Solar influences on climate have been discussed in a review by Gray et al. (2010) as well as in many places throughout the WG I contribution to AR5. The AR5 prediction discussed above was qualified also by noting that no allowance was made for secular changes in total solar irradiance. Although declining irradiance was cited in AR5 as a factor contributing to a recent slowdown in nearsurface warming, modulation of global-mean surface temperature by the eleven-year solar cycle was indicated to be under $0.1 \mathrm{~K}$ from solar maximum to minimum. Longer-term projections based on estimates of past variations are quite uncertain, but even if total solar irradiance were to decline from its $20^{\text {th }}$ century "grand maximum" to a "grand minimum" in the present century the resulting fall in the radiative forcing of surface-temperature change would be substantially smaller than the projected rise due to greenhouse-gas increases.

Variations in solar irradiance in the ultraviolet part of the spectrum are observed to be considerably larger than variations in total irradiance. Associated variations in ozone lead radiatively to a solar cycle in near-stratopause temperature that significantly modulates the cooling climatechange signal. Along with the evidence of a stratospheric influence on tropospheric predictability beyond the medium-range, this points to a need for continued measurement from space of spectrally-resolved solar irradiance.

\section{Conclusions}

Following general comment on modelling and data assimilation, the principal observational needs discussed in preceding sections are summarized. This is supplemented by some further discussion.

\subsection{Earth-system modelling and data assimilation}

Observations and their use with Earth-system models that couple atmospheric, oceanic, land and cryospheric processes, including biogeochemical components, have been the subject of this report. Increasing use is being made of such models in the study of climate change, but consideration here has been in the broader context of global environmental monitoring and prediction or projection for all time ranges out to a century or so ahead. Increasing demands and capabilities have brought global forecasting models for weather, atmospheric composition, land and ocean conditions to the point where they too can begin to be termed Earth-system models. A number of the challenges and approaches for improving models, and the associated observational needs, are common to the variants of Earth-system models used for monitoring, investigative studies, short-term forecasting and long-term projection.

Notwithstanding increasing integration and common interests among developers, a single path or priority route in the roadmap for the period ahead does not emerge from consideration of the range of model components and applications involved. Developmental aims such as expanding the modelling of ecosystems or integrating modelling of human systems and responses should not detract from continuing efforts to improve the core representations of dynamical and physical processes within models. For example, many aspects of the performance of Earth-system models depend on how well the hydrological cycle is represented, and basic improvements are still required 
in the modelling of the processes involved. There remains an important need for specialist modellers and for making the observations required to support their work.

Data assimilation continues to be developed with regard to the amounts of information extracted from particular sets of observations, the range of variables to which it is applied and the coupling of different Earth-system components. Further advances are expected over the period of the roadmap, concerning both use of new observations and improved use of past observations in reanalysis. More generally, whilst on a ten-year timescale there are goals and known applications for observations, it must also be recognized that the observations taken over the coming decade will be used many decades and centuries into the future for studying environmental change, in ways and with benefits that cannot be fully foreseen.

\subsection{Sustained long-term space-based observing systems}

Long-term space-based observation is provided by sequences of multi-satellite missions established for operational numerical weather prediction. The data provided by these missions have become more comprehensive over time, and are important also for climate and other applications. The data from polar orbit to be provided by the Chinese FY-3 series over the roadmap period should considerably enhance what is provided by continuing and developing European and US contributions.

The data from operational meteorological satellites are supplemented by data from other series of satellites. Particular examples are the data from scatterometers, optical imagers and microwave radiometers flown on a variety of platforms. Land-surface imagery is another type of measurement from space that has long-term continuity and substantial planned provision into the future. Longterm measurement of ocean surface topography has been provided by the sequence of Topex/Poseidon and Jason altimeters.

A substantial further enhancement of long-term observation will occur over the roadmap period with the full implementation of the Sentinel series of environmental missions. These provide continuation of past types of observation on an operational basis and additional capabilities such as improved resolution.

An important need is for confirmation of missions (or later launches within a multi-satellite programme) that are expected to provide continuity but are still classed as "planned" in mission databases, or for which there are still uncertainties concerning implementation. Full operational establishment of GNSS occultation is a need.

Laying the foundations for successor operational systems will become important later in the roadmap period.

\subsection{Other requirements for long-term space-based observations}

Notwithstanding an improving operational capability, there remain issues and uncertainties with regard to other types of observation that have proved to be useful, may be demonstrated to be useful by missions currently or soon to be in orbit, or for which good arguments have been advanced. 
A case that has been noted in several preceding sections is that of the looming dearth of limbsounding. This has been identified as a concern for several years. The requirement for long-term limb-scanning measurement from the upper troposphere to the stratopause was in particular identified in the 2010 Implementation Plan developed by the GCOS programme (GCOS, 2010). This requirement remains.

Also in the 2010 GCOS Implementation Plan, and likewise without much subsequent progress, was a reference mission providing measurements with absolute calibration traceable to SI standards, such as was expected from the CLARREO mission that is currently in an "extended pre-Phase A" state due to budgetary considerations. The strength of some of the case for this mission appears now to have weakened, as the shortening of the time-to-detection of climate change it offers relative to measurements by the hyperspectral sounders already in orbit (Wielicki et al., 2013) is being offset by the delay it has experienced. Moreover, the benefits it promised for the calibration of data from other satellites have been reduced due the inter-calibration enabled by the proven stable nature of measurements from several hyperspectral sounders and of GNSS occultation. Currently, NASA "continues to fund efforts to refine the mission design and to examine alternative platforms, such as the International Space Station, focusing on lower cost implementation while achieving a majority of the CLARREO science objectives" (clarreo.larc.nasa.gov/about-mission.html; February 2016).

The importance and challenges of modelling, monitoring and predicting the hydrological cycle necessitate long-term measurement of water vapour, cloud, precipitation, soil moisture and several other variables. Establishment of follow-on arrangements for the GPM mission will be required during the roadmap period. Observation of aerosols is also important, for initialising short-range predictions as well as for reducing the uncertainty in climate modelling. Improved observation from space will be provided by the operational meteorological and Sentinel systems. Nevertheless, the case for intermittent more-highly specified vertical-profiling research missions for the study of clouds and aerosol is likely to remain, and will need evaluating in the light of what has been learnt from recent and current missions and what will be learnt from the forthcoming EarthCARE mission.

Implementation of the proposed new generation of greenhouse-gas missions is another critical requirement for the roadmap period, particularly from the viewpoint of their combined use with ground-based observation for better estimation of source and sinks. The space-based contribution to monitoring the emissions of the fast-reacting gases that influence air quality and climate forcing would be served by spatial resolutions finer than currently planned, which should be explored further during the period.

Spectral monitoring of outgoing short- and long-wave radiation is also required in the above contexts, for diagnosing forcing and feedbacks associated with water vapour, cloud, aerosol and constituent gases. Spectrally-resolved short-wave as well as total solar irradiance is also important to measure, for its effects on the distribution of ozone in the upper stratosphere and mesosphere and consequently on radiative forcing. Continuity of these measurements needs to be ensured.

Several instances of the usefulness of measurements of the variations in gravity have been noted in preceding sections: for determination of the geoid and ocean bottom pressure, the amount of groundwater and the mass of ice sheets. Application to estimation of the flood potential of large river basins has also been reported (Reager et al., 2014). The GRACE follow-on mission should provide the opportunity to explore the potential for a highly desirable increase in spatial resolution 
through use of laser interferometry to measure variations in distance between its two component satellites, in addition to continuing the record provided by GRACE. It needs to be followed by higher spatial resolution measurement from missions such as GRACE-II, recommended by NRC (2007), or the proposed e.motion (Panet et al., 2013).

Gravimetry is just one type of relatively new or forthcoming measurement that has, or is expected, to provide information useful for monitoring and predicting changes to the Earth system. Examples of instruments in orbit whose value is currently being assessed include those measuring soil moisture, ocean salinity and sea-ice thickness, and new measurements of wind, surface water, biomass and fluorescence are expected in the first half of the roadmap period, as noted in section 3.6. Novel use of reflected GNSS signals to infer ocean- and land-surface properties (Yang et al., 2009; Yin et al., 2015) is one of the objectives of the GEROS-ISS mission (Martin-Neira et al., 2014; Wickert et al., 2014). Agility in mission planning will be needed to ensure prompt follow-on missions for types of observation that have been demonstrated to yield cost-effective benefits. Associated with this is the budgetary challenge of supporting routine measurement of an increasing number of key Earth-system variables while maintaining a vibrant programme of missions dedicated to answering research questions and demonstrating new measurement capabilities.

\subsection{Requirements for additional observations and socio-economic data}

The emphasis placed in this report on space-based observation of the Earth system should not detract from the importance also of in situ measurement and land-, sea- and air-based remote sensing. This includes the occasional undertaking of field programmes focussed on elucidating particular processes or for calibration and validation of space-based measurements, as well as systematic measurements from long-term networks. With regard to the latter, the networks of observing sites established for particular types of measurement vary considerably in their density and overall completeness of cover. Networks for precipitation and surface meteorological observations are relatively dense, but still inadequate to meets local needs for information in many parts of the world, in particular related to the impacts of and adaptation to climate variability and change.

The physical state of the surface and upper ocean is now quite comprehensively observed through buoys and profiling floats, although the declining status of the tropical moored-buoy network was an issue that prompted establishment of the TPOS 2020 project. Sensors are being developed for autonomous oceanic measurement of key biological and chemical variables. These will require widespread deployment, and observation also needs to be extended downward, through technological developments such as Deep Argo floats but also through ship-based measurement that can comprehensively sample a range of important variables. Observation also needs enhancing under sea ice.

Ground-based sampling of the atmosphere, the land and the fluxes between them from relatively sparse but well-instrumented networks making and processing both in situ and remote-sensing measurements to a high standard is essential. It needs to be sustained and expanded where necessary. Some decline in the number of stations and far from uniform geographical coverage in the networks for atmospheric composition is a particular concern. 
Much more detail on the current situation is contained in the review of in situ and remotely sensed observation of climate provided in the status report by GCOS (2015) prepared in parallel with this roadmap report. The GCOS report is being followed by an assessment by the GCOS programme of the required actions for inclusion in a new implementation plan in 2016. Ciais et al. (2014) provide a specific discussion and detailed recommendations for observation of the carbon cycle.

There is a need also for recovering past instrumental data and making them widely available. This involves in particular the scanning and digitization of paper records, but also the rehabilitation of some data held in digital form, such as from early satellite observations that have value as either input or evaluation data for reanalysis. Related data on land-use change, volcanic forcings, solar variations and so on are needed for use in climate models that are run to evaluate their performance over the past century or more, and for use in the assimilating models used to produce century-scale reanalyses. More generally, the products of data assimilation will be improved and become available for a wider range of variables as observations and models are improved in the ways foreseen in this roadmap. Data assimilation brings with it ancillary needs such as for good instrumental characterization for forward radiative transfer modelling, including for the past instruments whose data are used in reanalysis, and for good error characterization for data used in the form of retrievals. In turn, there is a need for assimilation centres to improve access to feedback information on the quality of both observational data and analysis products inferred from their processing.

Observation of ecosystems and biodiversity is generally not as well organized internationally as observation of the physical and chemical state of the Earth system. Here progress is expected during the roadmap period through initiatives that build on the concept of ECVs developed by GCOS. The Framework for Ocean Observing developed by Lindstrom et al. (2012) and its coordination processes are being organized around a set of Essential Ocean Variables (EOVs) that include but go beyond the oceanic ECVs. The EOVs will include variables related to ecosystems and biodiversity. Moreover, partners in the Group on Earth Observations Biodiversity Observation Network are developing and seeking consensus around a general set of Essential Biodiversity Variables aimed at providing the basis of monitoring programmes worldwide (Pereira et al., 2013).

Socio-economic data are a further requirement. International exchange and coordinated delivery mechanisms for such data are generally less developed than for data on the natural Earth system. One use for this type of data is in the development and operation of forecasting and climate services that use the data in conjunction with the outputs of Earth-system models. In such cases, services for national use may be developed by those that hold restricted data, but prompt exchange of data is required, nevertheless, if they are valuable for improving estimates of the emissions of pollutants that have transboundary effects, estimates that are needed for use in the regional and global models now being used in monitoring and forecasting. A broader set of data will need to be made openly available and readily accessible once modelling of human systems becomes integrated into Earthsystem models. Even if it takes some time for substantial progress to be achieved on the modelling side, data collection and stewardship should be put in place in advance, to support the development and evaluation of the required modelling.

The variables on which data are required include population, land use, land management and economic activity. Population data should be spatially disaggregated to the minimum census unit (typically of the order of a few tens of kilometres) by gender, age, health, wealth and education. 
Land-use and land-management data need to be made available with better temporal as well as spatial resolution. Space-based data have a role to play here, but in situ survey data are required as well, to quantify both inputs to the land, such as fertilizer, tillage, irrigation and pesticides, and the removals of yields. Land management also includes information on forestry practices. Economic activity for both goods and services, such as prices, quantities traded and destinations of export, are the staple input and validation data for the economic models which underpin most human-system projections in conjunction with demographic models. These data tend currently to be available only at national scale, and as with the other types of data they need to be more spatially disaggregated.

\subsection{New approaches to observation}

Technological development underlies the various advances in satellite and in situ observation discussed already in this report. It also continues to provide opportunities for new approaches to making observations. Some are mentioned here.

A particular development in space-based observation involves the use of smaller satellites. Socalled microsatellites such as ESA's PROBA series have already found application in imaging the surface of the Earth, with monitoring of vegetation the focus of the latest mission to launch, PROBAV. Smaller still are the cubesats, built with standard dimensions based on one or several $10 \mathrm{~cm}$-sided cubical units (www.esa.int/Education/CubeSats_and_Education_the_Fly_Your_Satellite!_programme; cubesat.jpl.nasa.gov/projects.html; www.qb50.eu/index.php). Examples of forthcoming missions related to earth observation are PICASSO for measurement of stratospheric ozone and mesospheric temperature, RAVAN and SIMBA for the radiation budget and TEMPEST-D for clouds and precipitation. The RACE cubesat for water vapour measurement was lost due to launch failure late in 2014, but the GRIFEX cubesat contributing to detector development for measurement of air quality and ocean colour from geostationary orbit was deployed in space early in 2015. The potential for operating comprehensive measurement systems based on constellations of cubesats is being assessed.

As regards in situ observation, deployment close to the Earth's surface of the modern generation of small unmanned aerial vehicles, or drones, is being explored for measurement of several types of variable. This includes ecological observation (Koh and Wich, 2012; Anderson and Gaston, 2013), meteorological and air-quality observation in the lower part of the planetary boundary layer (Illingworth et al., 2014), volcano monitoring (Diaz et al., 2012, Amici et al., 2013) and imagery to support urban drainage modelling (Tokarczyk et al., 2015). Wireless sensor networks similarly have multiple developing applications for local monitoring. Particular examples are the implementation using calibrated radiation sensors undertaken in the context of developing reference measurement of FAPAR, under the auspices of the Land Product Validation Subgroup of the CEOS Working Group on Calibration and Validation (Ipvs.gsfc.nasa.gov/Fpar_home.html), and the deployment of low-cost temperature sensors for urban measurement, using proprietary Wi-Fi networks (Chapman et al., 2015). Consideration has also been given to the potential for surface atmospheric observations from motor vehicles (Mahoney and O'Sullivan, 2013) and smartphones (Mass and Madaus, 2014). Stratospheric wind data may become available in future from balloons with active level-control being developed in Google's Project Loon. 


\subsection{Data management and high-end computing}

Technological (as well as institutional) issues have to be faced in handling the ever-increasing volume of global observational data and related model data needed to support integrated Earthsystem science and its applications. This is one of the biggest of the "Big Data" challenges, particularly in view of the diversity of the data involved. The requirement is for databases of observations and the products derived from them that are easy to discover and access, that provide data with structures and in formats that are easy to use, and that are linked to information on the perceived quality of the observations and fitness-for-purpose of the products, something which itself does not come free of development and information-technology costs. Data also have to be readily available for time-critical applications, be they connected with shorter-term forecasting or the demanding production schedule for longer-term reanalyses, and archival systems have to cope with the rate at which derived data are produced by such applications. Past data may be useful long into the future and require stewardship even if the volumes of data are small compared with those produced by current observing systems and applications. Capacities will need to keep pace with future growth in the rates at which both observational and derived data are produced. A greater degree of on-demand construction of user-specific products, for example through use of cloud computing, is also envisaged.

Information on the Earth system can be improved by exploiting the data provided by better observing systems, but can also be improved by better extraction of information from the data provided by past and present observing systems. This comes in part from the occasional recalibration of data.

By some measures at least, the major part of the improvement in numerical weather prediction over the past thirty-five years has come from better data assimilation and modelling rather than from improvements in observing systems, considerable though some of these have been (Dee et al., 2014). Better data assimilation and modelling in general require greater levels of computing, and the associated expenditure may have to rise more substantially than in the past if progress is to be maintained and the benefits of the even larger investments made in observation more fully realized. Here the challenge is to exploit effectively high-performance computer systems that make everincreasing use of parallel processing, as the growth provided rather inexpensively in the past by increases in single-processor computing performance can no longer be sustained. This comes to the fore in Earth-system data assimilation and the shorter-term global forecasting applications that use the highest resolution and where time is of the essence for the delivery of results. There is otherwise more scope for scheduling workload in parallel.

\subsection{Enhancement of international co-operation}

The existing mechanisms for international co-operation provide global fora for coordination aimed at achieving a comprehensive overall system of systems for Earth observation, with complementarity among its component systems. Nevertheless, much more remains to be done to ensure that resources are used to greatest effect in meeting the various needs for observationally based information on the Earth system.

Coordination among the providers of operational meteorological satellites has a long and commendable history, and continues to broaden. The CEOS virtual constellations are a promising 
approach that has already proved in some cases to be an efficient route towards enhanced international co-operation and optimal use of world resources in terms of funding and expertise. Also important is the strategic development of the Architecture for Climate Monitoring from Space led by CEOS, CGMS and WMO. It includes consideration of what is needed for ground-based data systems, including reprocessing, inter-calibration and product generation, where existing successful initiatives such as GSICS, SCOPE-CM and the ESA CCI need sustaining and developing, as does cooperation among the data providers and producers of reanalyses. The extent and timeliness of international data exchange is improving or in prospect under initiatives such as GEO, Copernicus and the Global Framework for Climate Services, but much more remains to be done. Continued cooperation is also needed to protect all parts of the radio frequency spectrum used to measure, collect and disseminate Earth observation data. The bands used for passive sensing require particular vigilance.

Despite what has been achieved, the OSCAR and CEOS databases show several gaps and apparent redundancies in current and planned future provision of Earth observation from space, highlighting the scope for improved co-operation among agencies worldwide. Limitations to international cooperation include lack of data sharing and otherwise undue costs and delays in data access. They also result from political obstacles such as the ITAR restrictions on technology. Considerable resources are consequently deployed in duplicating developmental effort and systems.

Increased international co-operation is a vital means to expand the scope of Earth observation through the reduction of unnecessary redundancies, the sharing of the burden among a larger global base and the redeployment of the freed effort and money into observational sectors where planned provision is inadequate, if not into meeting the increasing costs of data handling and highperformance computing. Extending international co-operation has proven extremely beneficial in other domains of space research, such as planetary exploration and human space flight, as demonstrated by the experience of COSPAR over what is approaching sixty years of social, economic, political, scientific and technological change. It is of utmost importance that the barriers to further extension of co-operation be rapidly removed.

\section{Acknowledgments}

This is the report of a study team comprising core members selected by COSPAR, supplemented by members of COSPAR Scientific Commission A and the Executive Director of COSPAR. The leader of the study team, Adrian Simmons, prepared the report with the assistance of Jean-Louis Fellous, Venkatachalam Ramaswamy and Kevin Trenberth, who contributed both through provision of text and editorially. The report draws on textual inputs by other team members, and on subsequent comments provided by team members, community and journal reviewers and the journal editor. A particularly extensive and helpful review was provided by Bjorn Stevens (MPI for Meteorology).

\section{References}

Ablain, M., A. Cazenave, G. Larnicol, M. Balmaseda, P. Cipollini, Y. Faugère, M.J. Fernandes, O. Henry, J.A. Johannessen, P. Knudsen, O. Andersen, J. Legeais, B. Meyssignac, N. Picot, M. Roca, S. Rudenko, M.G. Scharffenberg, D. Stammer, G. Timms and J. Benveniste, 2015. Improved sea level record over 
the satellite altimetry era (1993-2010) from the Climate Change Initiative Project. Ocean Sci.,11, 2029-2071, doi:10.5194/os-11-67-2015

Ackerman, T.P., and G.M. Stokes, 2003. The Atmospheric Radiation Measurement Program. Phys. Today, 56, 38-44, doi: 10.1063/1.1554135

Agustí-Panareda, A., S. Massart, F. Chevallier, S. Boussetta, G. Balsamo, A. Beljaars, P. Ciais, N.M. Deutscher, R. Engelen, L. Jones, R. Kivi, J.-D. Paris, V.-H. Peuch, V. Sherlock, A.T. Vermeulen, P.O. Wennberg and D. Wunch, 2014. Forecasting global atmospheric $\mathrm{CO}_{2}$. Atmos. Chem. Phys., 14, 1195911983, doi:10.5194/acp-14-11959-2014

Albergel, C., P. de Rosnay, G. Balsamo, L. Isaksen and J. Muñoz-Sabater, 2012. Soil Moisture Analyses at ECMWF: Evaluation using global ground-based in situ observations. J. Hydrometeor, 13, 14421460, doi: /10.1175/JHM-D-11-0107.1

Albergel, C., E. Dutra, J. Muñoz-Sabater, T. Haiden, G. Balsamo, A. Beljaars, L. Isaksen, P. de Rosnay, I. Sandu and N. Wedi, 2015. Soil temperature at ECMWF: an assessment using ground-based observations. J. Geophys. Res., 120(4), 1361-1373, doi: 10.1002/2014JD022505

Alexe, M., P. Bergamaschi, A. Segers, R. Detmers, A. Butz, O. Hasekamp, S. Guerlet, R. Parker, H. Boesch, C. Frankenberg, R.A. Scheepmaker, E. Dlugokencky, C. Sweeney, S.C. Wofsy and E.A. Kort, 2014. Inverse modeling of $\mathrm{CH}_{4}$ emissions for 2010-2011 using different satellite retrieval products from GOSAT and SCIAMACHY. Atmos. Chem. Phys., 15, 113-133, doi: 10.5194/acp-15-113-2015, 2015.

Allen, M.R., D.J. Frame, C. Huntingford, C.D. Jones, J.A. Lowe, M. Meinshausen and N. Meinshausen, 2009. Warming caused by cumulative carbon emissions towards the trillionth tonne. Nature, 458 , 1163-1166, doi: 10.1038/nature08019

Al-Yaari, A., J.-P. Wigneron, A. Ducharne, Y. Kerr, P. de Rosnay, R. de Jeu, A. Govind, A. Al Bitar, C. Albergel, J. Muñoz-Sabater, P. Richaume and A. Mialon, 2014. Global-scale evaluation of two satellite-based passive microwave soil moisture datasets (SMOS and AMSR-E) with respect to Land Data Assimilation System estimates. Remote Sens. Environ., 149, 181-195, doi:

10.1016/j.rse.2014.04.006

Amici, S., M. Turci, S. Giammanco, L. Spampinato and F. Giulietti, 2013. UAV Thermal Infrared Remote Sensing of an Italian Mud Volcano. Advances in Remote Sensing, 2, 358-364, doi: 10.4236/ars.2013.24038

Anderson, K., and K.J. Gaston, 2013. Lightweight unmanned aerial vehicles will revolutionize spatial ecology. Front Ecol Environ, 11(3), 138-146, doi: 10.1890/120150

Balmaseda, M.A., D. Dee, A. Vidard and D.L.T. Anderson, 2007. A multivariate treatment of bias for sequential data assimilation: Application to the tropical oceans. Q.J.R. Meteorol. Soc., 133, 167-179, doi: 10.1002/qj.12

Balmaseda, M.A., F. Yosuke, O. Alves, T. Lee, M. Rienecker, A. Rosati, D. Stammer, Y. Xue, H. Freeland, M.J. McPhaden, L. Goddard and C. Coelho, 2010. Role of the Ocean Observing System in an End-to-End Seasonal Forecasting System. In Proceedings of OceanObs'09: Sustained Ocean 
Observations and Information for Society (Vol. 1), Venice, Italy, 21-25 September 2009, Hall, J., Harrison, D.E. \& Stammer, D., Eds., ESA Publication WPP-306, doi:10.5270/OceanObs09.pp.03

Balmaseda, M.A., K. Mogensen and A.T. Weaver, 2013a. Evaluation of the ECMWF ocean reanalysis system ORAS4. Q.J.R. Meteorol. Soc., 139, 1132-1161, doi: 10.1002/qj.2063

Balmaseda, M.A., K.E. Trenberth and E. Källén, 2013b. Distinctive climate signals in reanalysis of global ocean heat content. Geophys. Res. Lett., 40, 1754-1759, doi:10.1002/grl.50382

Balsamo, G., A. Agustì-Panareda, C. Albergel, A. Beljaars, S. Boussetta, E. Dutra, T. Komori, S. Lang, J. Muñoz-Sabater, F. Pappenberger, P. de Rosnay, I. Sandu, N. Wedi, A. Weisheimer, F. Wetterhall, E. Zsoter, 2014. Representing the Earth surfaces in the Integrated Forecasting System: Recent advances and future challenges. ECMWF Tech. Memo., 729, 48pp. Available from www.ecmwf.int

Balsamo, G., C. Albergel, A. Beljaars, S. Boussetta, E. Brun, H. Cloke, D. Dee, E. Dutra, J. Muñoz Sabater, F. Pappenberger, P. de Rosnay, T. Stockdale and F. Vitart, 2015. ERA-Interim/Land: A global land-surface reanalysis data set. Hydrol. Earth Syst. Sci., 19, 389-407, doi: 10.5194/hess-19-389-2015

Basu, S., S. Guerlet, A. Butz, S. Houweling, O. Hasekamp, I. Aben, P. Krummel, P. Steele, R. Langenfelds, M.Torn, S. Biraud, B. Stephens, A. Andrews and D. Worthy, 2013. Global CO2 fluxes estimated from GOSAT retrievals of total column $\mathrm{CO}_{2}$. Atmos. Chem. Phys., 13, 8695-8717, doi: 10.5194/acp-13-8695-2013

Bechtold, P., N. Semane, P. Lopez, J.-P. Chaboureau, A. Beljaars, N. Bormann, 2014. Representing equilibrium and nonequilibrium convection in large-scale models. J. Atmos. Sci., 71, 734-753, doi: 10.1175/JAS-D-13-0163.1

Becker, A., P. Finger, A. Meyer-Christoffer, B. Rudolf, K. Schamm, U. Schneider and M. Ziese, 2013. A description of the global land-surface precipitation data products of the Global Precipitation Climatology Centre with sample applications including centennial (trend) analysis from 1901present. Earth Syst. Sci. Data, 5, 71-99, doi: 10.5194/essd-5-71-2013

Beer, C., M. Reichstein, E. Tomelleri, P. Ciais, M. Jung, N. Carvalhais, C. Rödenbeck, M.A. Arain, D. Baldocchi, G.B. Bonan, A. Bondeau, A. Cescatti, G. Lasslop, A. Lindroth, M. Lomas, S. Luyssaert, H. Margolis, K.W. Oleson, O. Roupsard, E. Veenendaal, N. Viovy, C. Williams, F.I. Woodward and D. Papale, 2010. Terrestrial gross carbon dioxide uptake: Global distribution and covariation with climate. Science, 329, 834-838, doi: 10.1126/science.1184984

Behrangi, A., G. Stephens, R.F. Adler, G.J. Huffman, B. Lambrigtsen and M.Lebsock, 2014. An Update on the Oceanic Precipitation Rate and Its Zonal Distribution in Light of Advanced Observations from Space. J. Climate, 27, 3957-3965. doi: 10.1175/JCLI-D-13-00679.1

Bell, M.J., M.J. Martin and N.K. Nichols, 2004. Assimilation of data into an ocean model with systematic errors near the equator. Q.J.R. Meteorol. Soc., 130, 873-893, doi: 10.1256/qj.02.109

Bellouin, N., J. Quaas, J.-J. Morcrette and O. Boucher, 2013. Estimates of aerosol radiative forcing from the MACC re-analysis. Atmos. Chem. Phys., 13, 2045-2062, doi: 10.5194/acp-13-2045-2013 
Bergamaschi, P., S. Houweling, A. Segers, M. Krol, C. Frankenberg, R.A. Scheepmaker, E. Dlugokencky, S.C. Wofsy, E.A. Kort, C. Sweeney, T. Schuck, C. Brenninkmeijer, H. Chen, V. Beck and C. Gerbig, 2013. Atmospheric $\mathrm{CH}_{4}$ in the first decade of the 21st century: Inverse modeling analysis using SCIAMACHY satellite retrievals and NOAA surface measurements. J. Geophys. Res. Atmos., 118, 7350-7369, doi:10.1002/jgrd.50480

Berger, M., J. Moreno, J.A. Johannessen, P.F. Levelt and R.F. Hanssen, 2012. ESA's sentinel missions in support of Earth system science. Remote Sens. Environ., 120, 84-90, doi:

10.1016/j.rse.2011.07.023.

Berrisford, P., P. Kållberg, S. Kobayashi, D. Dee, S. Uppala, A.J. Simmons, P. Poli and H. Sato, 2011. Atmospheric conservation properties in ERA-Interim. Quart. J. Roy. Meteor. Soc., 137, 1381-1399, doi: $10.1002 / q j .864$

Bodeker, G.E., S. Bojinski, D. Cimini, R.J. Dirksen, M. Haeffelin, J.W. Hannigan, D.F. Hurst, T. Leblanc, F. Madonna, M. Maturilli, A.C. Mikalsen, R. Philipona, T. Reale, D.J. Seidel, D.G.H. Tan, P.W. Thorne, H. Vömel and J. Wang, 2015. Reference upper-air observations for climate: From concept to reality. Bull. Amer. Meteor. Soc., e-View, doi: 10.1175/BAMS-D-14-00072.1

Bojinski, S., M. Verstraete, T.C. Peterson, C. Richter, A. Simmons and M. Zemp, 2014. The concept of Essential Climate Variables in support of climate research, applications, and policy. Bull. Amer. Meteor. Soc., 95, 1431-1443, doi: 10.1175/BAMS-D-13-00047.1

Bony,S., B. Stevens, I.H. Held, J.F. Mitchell, J.-L. Dufresne, K.A. Emanuel, P. Friedlingstein, S. Griffies and C. Senior, 2013. Carbon dioxide and climate: Perspectives on a scientific assessment. In Climate Science for Serving Society: Research, Modelling and Prediction Priorities, Eds. Hurrell, J.W. \& Asrar, G.) 391-413 (Springer, 2013)

Bony, S., B. Stevens, D.M.W. Frierson, C. Jakob, M. Kageyama, R. Pincus, T.G. Shepherd, S.C. Sherwood, A.P. Siebesma, A.H. Sobel, M. Watanabe and M.J. Webb, 2015. Clouds, circulation and climate sensitivity. Nature Geosci., 8, 261-268, doi: 10.1038/NGEO2398

Borde, R., O. Hautecoeur and M. Carranza, 2016. EUMETSAT Global AVHRR Wind Product. J. Atmos. Ocean. Tech., in press, doi: 10.1175/JTECH-D-15-0155.1

Bosilovich, M.G., F.R. Robertson and J. Chen, 2011. Global energy and water budgets in MERRA. J. Clim. 24, 5721-5739, doi: 10.1175/2011JCLI4175.1

Boucher, C., M. Pearlman and P. Sarli, 2015. Global geodetic observatories. Adv. Space Res., 55(1), 24-39, doi: 10.1016/j.asr.2014.10.011

Boussetta, S., G. Balsamo, A. Beljaars, T. Kral and L. Jarlan, 2013a. Impact of a satellite-derived Leaf Area Index monthly climatology in a global Numerical Weather Prediction model. Int. J. Rem. Sens., 34, 3520-3542, doi: 10.1080/01431161.2012.716543

Boussetta, S., G. Balsamo, A. Beljaars, A. Agustí-Panareda, J.-C. Calvet, C., Jacobs, B. van den Hurk, P. Viterbo, S. Lafont, E. Dutra, L. Jarlan, M. Balzarolo, D. Papale and G. van der Werf, 2013b. Natural land carbon dioxide exchanges in the ECMWF integrated forecasting system: Implementation and offline validation. J. Geophys. Res. Atmos., 118, 5923-5946, doi:10.1002/jgrd.50488 
Boussetta, S., G. Balsamo, E. Dutra, A. Beljaars and C. Albergel, 2015. Analysis of surface albedo and Leaf Area Index from satellite observations and their impact on numerical weather prediction. Remote Sens. Environ., 163, 111-126, doi: 10.1016/j.rse.2015.03.009

Bréon, F.-M., and P. Ciais, 2010. Spaceborne remote sensing of greenhouse gas concentrations. Comptes Rendus Geoscience, 342, 412-424, doi: 10.1016/j.crte.2009.09.012

Brocca, L., F. Melone, T. Moramarco, W. Wagner, V. Naeimi, Z. Bartalis and S. Hasenauer, 2010. Improving runoff prediction through the assimilation of the ASCAT soil moisture product. Hydrol. Earth Syst. Sci., 14, 1881-1893, doi :10.5194/hess-14-1881-2010

Bryan, K., S. Manabe and R.C. Pacanowski, 1975. A global ocean-atmosphere climate model. Part II. The oceanic circulation. J. Phys. Oceanogr., 5, 30-46, doi: 10.1175/1520-

0485(1975)005<0030:AGOACM>2.0.CO;2

Burgers, G., M.A. Balmaseda, F.C. Vossepoel, G.J. van Oldenborgh and P.J. van Leeuwen, 2002. Balanced Ocean-Data Assimilation near the Equator. J. Phys. Oceanogr., 32, 2509-2519, doi: 10.1175/1520-0485-32.9.2509

Butler, D., 2014. Earth Observation enters next phase. Nature, 508, p160-161

Cariolle, D. and M. Déqué, 1986. Southern-hemisphere medium-scale waves and total ozone disturbances in a spectral general circulation model. J. Geophys. Res., 91, 10825-10846, doi: 10.1029/JD091iD10p10825

Carlslaw, K.S., L.A. Lee, C.L. Reddington, K.J. Pringle, A. Rap, P.M. Forster, G.W. Mann, D.V. Spracklen, M.T. Woodhouse, L.A. Regayre and J.R. Pierce, 2013. Large contribution of natural aerosols to uncertainty in indirect forcing. Nature, 503, 67-71, doi: 10.1038/nature12674

Chapman, L., C.L. Muller, D.T. Young, E.L. Warren, C.S.B. Grimmond, X.-M. Cai and E.J.S. Ferranti, 2015. The Birmingham Urban Climate Laboratory: An Open Meteorological Test Bed and Challenges of the Smart City. Bull. Amer. Meteor. Soc., 96, 1545-1560, doi: 10.1175/BAMS-D-13-00193.1

Chepurin, G. A., J. A. Carton and E. Leuliette, 2014. Sea level in ocean reanalyses and tide gauges. J. Geophys. Res. Oceans, 119, 147-155, doi: 10.1002/2013JC009365

Chevallier, F., N. Deutscher, T.J. Conway, P. Ciais, L. Ciattaglia, S. Dohe, M. Fröhlich, A.J. GomezPelaez, D. Griffith, F. Hase, L. Haszpra, P. Krummel, E. Kyrö, C. Labuschagne, R. Langenfelds, T. Machida, F. Maignan, H. Matsueda, I. Morino, J. Notholt, M. Ramonet, Y. Sawa, M. Schmidt, V. Sherlock, P. Steele, K. Strong, R. Sussmann, P. Wennberg, S. Wofsy, D. Worthy, D. Wunch and M. Zimnoch, 2011. Global $\mathrm{CO}_{2}$ fluxes inferred from surface air-sample measurements and from TCCON retrievals of the $\mathrm{CO}_{2}$ total column. Geophys. Res. Lett., 38, L24810, doi: 10.1029/2011GL049899

Church, J.A., P.U. Clark, A. Cazenave, J.M. Gregory, S. Jevrejeva, A. Levermann, M.A. Merrifield, G.A. Milne, R.S. Nerem, P.D. Nunn, A.J. Payne, W.T. Pfeffer, D. Stammer and A.S. Unnikrishnan, 2013. Sea Level Change. In: Climate Change 2013: The Physical Science Basis. Contribution of Working Group I to the Fifth Assessment Report of the Intergovernmental Panel on Climate Change [Stocker, T.F., D. Qin, G.,-K. Plattner, M. Tignor, S.K. Allen, J. Boschung, A. Nauels, Y. Xia, V. Bex and P.M. Midgley (eds.)]. Cambridge University Press, Cambridge, United Kingdom and New York, NY, USA. 
Ciais, P., A.J. Dolman, R. Dargaville, L. Barrie, A. Bombelli, J.Butler, P. Canadell and T. Moriyama, 2010. Geo Carbon Strategy, GEO Secretariat, Geneva / FAO, Rome, 48 pp.

Ciais, P., C. Sabine, G. Bala, L. Bopp, V. Brovkin, J. Canadell, A. Chhabra, R. DeFries, J. Galloway, M. Heimann, C. Jones, C. Le Quéré, R.B. Myneni, S. Piao and P. Thornton, 2013. Carbon and Other Biogeochemical Cycles. In: Climate Change 2013: The Physical Science Basis. Contribution of Working Group I to the Fifth Assessment Report of the Intergovernmental Panel on Climate Change [Stocker, T.F., D. Qin, G.-K. Plattner, M. Tignor, S.K. Allen, J. Boschung, A. Nauels, Y. Xia, V. Bex and P.M. Midgley (eds.)]. Cambridge University Press, Cambridge, United Kingdom and New York, NY, USA.

Ciais, P., A.J. Dolman, A. Bombelli, R. Duren, A. Peregon, P.J. Rayner, C. Miller, N. Gobron, G. Kinderman, G. Marland, N. Gruber, F. Chevallier, R.J. Andres, G. Balsamo, L. Bopp, F.-M. Bréon, G. Broquet, R. Dargaville, T.J. Battin, A. Borges, H. Bovensmann, M. Buchwitz, J. Butler, J.G. Canadell, R.B. Cook, R. DeFries, R. Engelen, K.R. Gurney, C. Heinze, M. Heimann, A. Held, M. Henry, B. Law, S. Luyssaert, J. Miller, T. Moriyama, C. Moulin, R.B. Myneni, C. Nussli, M. Obersteiner, D. Ojima, Y. Pan, J.-D. Paris, S.L. Piao, B. Poulter, S. Plummer, S. Quegan, P. Raymond, M. Reichstein, L. Rivier, C. Sabine, D. Schimel, O. Tarasova, R. Valentini, R. Wang, G. van der Werf, D. Wickland, M. Williams and C. Zehner, 2014. Current systematic carbon-cycle observations and the need for implementing a policy-relevant carbon observing system. Biogeosciences, 11, 3547-3602, doi: 10.5194/bg-11-35472014

Ciavatta, S., R. Torres, V. Martinez-Vicente, T. Smyth, G. Dall'Olmo, L. Polimene and J.I. Allen, 2014. Assimilation of remotely-sensed optical properties to improve marine biogeochemistry modelling, Progress in Oceanography, 127, September 2014, Pages 74-95, doi: 10.1016/j.pocean.2014.06.002

Collins, M., R. Knutti, J. Arblaster, J.-L. Dufresne, T. Fichefet, P. Friedlingstein, X. Gao, W.J. Gutowski, T. Johns, G. Krinner, M. Shongwe, C. Tebaldi, A.J. Weaver and M. Wehner, 2013. Long-term Climate Change: Projections, Commitments and Irreversibility. In: Climate Change 2013: The Physical Science Basis. Contribution of Working Group I to the Fifth Assessment Report of the Intergovernmental Panel on Climate Change [Stocker, T.F., D. Qin, G.-K. Plattner, M. Tignor, S.K. Allen, J. Boschung, A. Nauels, Y. Xia, V. Bex and P.M. Midgley (eds.)]. Cambridge University Press, Cambridge, United Kingdom and New York, NY, USA.

Collins, W.D., V. Ramaswamy, M.D. Schwarzkopf, Y. Sun, R.W. Portmann, Q. Fu, S.E.B. Casanova, J.-L. Dufresne, D.W. Fillmore, P.M.D. Forster, V.Y. Galin, L.K. Gohar, W.J. Ingram, D.P. Kratz, M.-P. Lefebvre, J. Li, P. Marquet, V. Oinas, Y. Tsushima, T. Uchiyama and W.Y. Zhong, 2006. Radiative forcing by well-mixed greenhouse gases: Estimates from climate models in the Intergovernmental Panel on Climate Change (IPCC) Fourth Assessment Report (AR4), J. Geophys. Res., 111, D14317, doi: 10.1029/2005JD006713

Compo, G.P., J.S. Whitaker, P.D. Sardeshmukh, N. Matsui, R.J. Allan, X. Yin, B.E. Gleason, R.S. Vose, G. Rutledge, P. Bessemoulin, S. Brönnimann, M. Brunet,R.I. Crouthamel, A.N. Grant, P.Y. Groisman, P.D. Jones, M.C. Kruk, A.C. Kruger, G.J. Marshall, M. Maugeri, H.Y. Mok, $\varnothing$. Nordli, T.F. Ross, R.M. Trigo, X.L. Wang, S.D. Woodruff and S.J. Worley, 2011. The Twentieth Century Reanalysis Project. Q.J.R. Meteorol. Soc., 137, 1-28, doi: 10.1002/qj.776 
Courtier, P., J.-N. Thépaut and A. Hollingsworth, 1994. A strategy for operational implementation of 4D-Var, using an incremental approach. Quart. J. Roy. Meteor. Soc., 120, 1367-1387, doi:

10.1002/qj.49712051912

de Boisséson, E., M.A. Balmaseda, S. Abdalla, E. Källén and P.A.E.M. Janssen, 2014. How robust is the recent strengthening of the Tropical Pacific trade winds? Geophys. Res. Lett., 41, 4398-4405, doi: 10.1002/2014GL060257

Dee, D.P., 2005. Bias and data assimilation. Q.J.R. Meteorol. Soc., 131, 3323-3343, doi: 10.1256/qj.05.137

Dee, D.P., and S. Uppala, 2009. Variational bias correction of satellite radiance data in the ERAInterim reanalysis. Q. J. R. Meteorol. Soc. 135, 1830-1841, doi: 10.1002/qj.493

Dee, D.P., S.M. Uppala, A.J. Simmons, P. Berrisford, P. Poli, S. Kobayashi, U. Andrae3 M.A.

Balmaseda, G. Balsamo, P. Bauer, P. Bechtold, A.C.M. Beljaars, L. van de Berg, J. Bidlot, N. Bormann, C. Delsol, R. Dragani, M. Fuentes, A.J. Geer, L. Haimberger, S.B. Healy, H. Hersbach, E.V. Hólm, L. Isaksen, P. Kållberg, M. Köhler, M. Matricardi, A.P. McNally, B.M. Monge-Sanz, J.-J. Morcrette, B.-K. Park, C. Peubey, P. de Rosnay, C. Tavolato, J.-N. Thépaut and F. Vitart, 2011. The ERA-Interim reanalysis: configuration and performance of the data assimilation system. Q.J.R. Meteor. Soc., 137, 553-597, doi: 10.1002/qj.828

Dee, D.P., M. Balmaseda, G. Balsamo, R. Engelen, A.J. Simmons and J.-N. Thépaut, 2014. Toward a consistent reanalysis of the climate system. Bull. Am. Meteorol. Soc.. 95, 1235-1248, doi: 10.1175/BAMS-D-13-00043.1.

Delworth, T.L., A. Rosati, W. Anderson, A.J. Adcroft, V. Balaji, R. Benson, K. Dixon, S.M. Griffies, H.-C. Lee, R.C. Pacanowski, G.A. Vecchi, A.T. Wittenberg, F. Zeng and R. Zhang, 2012. Simulated Climate and Climate Change in the GFDL CM2.5 High-Resolution Coupled Climate Model. J. Climate, 25, 2755-2781, doi: 10.1175/JCLI-D-11-00316.1

Derber J.C., and W.-S. Wu, 1998. The use of TOVS cloud-cleared radiances in the NCEP SSI analysis system. Mon. Weather Rev., 126, 2287-2299, doi: 10.1175/1520-

0493(1998)126<2287:TUOTCC>2.0.CO;2

de Rosnay, P., M. Drusch, D. Vasiljevic, G. Balsamo, C. Albergel and L. Isaksen, 2013. A simplified Extended Kalman Filter for the global operational soil moisture analysis at ECMWF. Q.J.R. Meteorol. Soc., 139, 1199-1213, doi: 10.1002/qj.2023

de Rosnay, P., G. Balsamo, C. Albergel, J. Muñoz-Sabater and L. Isaksen, 2014. Initialisation of land surface variables for numerical weather prediction. Surveys in Geophysics, 35, 607-621, doi: $10.1007 / \mathrm{s} 10712-012-9207-\mathrm{x}$

Dharssi, I., K.J. Bovis, B. Macpherson and C.P. Jones, 2011. Operational assimilation of ASCAT surface soil wetness at the Met Office. Hydrol. Earth Syst. Sci., 15, 2729-2746, doi: 10.5194/hess-15-27292011 
Diaz, J., E. Corrales, Y. Madrigal, D. Pieri, G. Bland, T. Miles and M. Fladeland, 2012. Volcano monitoring with small unmanned aerial systems. Infotech@Aerospace 2012, American Institute of Aeronautics and Astronautics. doi: 10.2514/6.2012-2522

Doherty, S.J., S.G. Warren, T.C. Grenfell, A.D. Clarke and R.E. Brandt, 2010. Light-absorbing impurities in Arctic snow. Atmos. Chem. Phys., 10, 11647-11680, doi: 10.5194/acp-10-11647-2010

Dorigo, W.A., W. Wagner, R. Hohensinn, S. Hahn, C. Paulik, A. Xaver, A. Gruber, M. Drusch, S. Mecklenburg, P. van Oevelen, A. Robock and T. Jackson, 2011a. The International Soil Moisture Network: A data hosting facility for global in situ soil moisture measurements. Hydrol. Earth Syst. Sci., 15, 1675-1698, doi: 10.5194/hess-15-1675-2011

Dorigo, W., P. Van Oevelen, W. Wagner, M. Drusch, S. Mecklenburg, A. Robock and T. Jackson, 2011b. A new international network for in situ soil moisture data. EOS 92 (17), pp. 141-142, doi: 10.1029/2011EO170001

Dorigo, W.A., R.A.M. de Jeu, D. Chung, R.M. Parinussa, Y.Y. Liu, W. Wagner and D. Fernandez-Prieto, 2012. Evaluating global trends (1988-2010) in harmonized multi-satellite surface soil moisture. Geophys. Res. Lett. 39.18, doi: 10.1029/2012GL052988

Dowell, M., P. Lecomte, R. Husband, J. Schulz, T. Mohr, Y. Tahara, R. Eckman, E. Lindstrom, C. Wooldridge, S. Hilding, J.Bates, B. Ryan, J. Lafeuille and S. Bojinski, 2013. Strategy Towards an Architecture for Climate Monitoring from Space. Pp. 39. Available from: www.ceos.org; www.wmo.int/sat; www.cgms-info.org/

Drinkwater, M.R., P. Silvestrin and M. Borgeaud, 2012. ESA's Earth Explorer scientific missions. IGARSS, 2012 IEEE International, pp. 5526-5529, doi: 10.1109/IGARSS.2012.6352354

Dumont, M., E. Brun, G. Picard, M. Michou, Q. Libois, J-R. Petit, M. Geyer, S. Morin and B. Josse, 2014. Contribution of light-absorbing impurities in snow to Greenland's darkening since 2009. Nature Geosci., 7, 509-512, doi: 10.1038/ngeo2180

Elbern, H., A. Strunk, H. Schmidt and O. Talagrand, 2007. Emission rate and chemical state estimation by 4-dimensional variational inversion. Atmos. Chem. Phys., 7, 3749-3769, doi: 10.5194/acp-7-3749-2007

Entekhabi, D., S. Yueh, P. O'Neill, K. Kellogg et al., 2014. SMAP Handbook, JPL Publication JPL 4001567, Jet Propulsion Laboratory, Pasadena, California, 182 pages. Available from smap.jpl.nasa.gov

Eyre, J.R., and P.P. Weston, 2014. The impact of the temporal spacing of observations on analysis errors in an idealised data assimilation system. Q.J.R. Meteorol. Soc., 140, 1441-1452, doi: 10.1002/qj.2227

Eyring, V., M. Righi, M. Evaldsson, A. Lauer, S. Wenzel, C. Jones, A. Anav, O. Andrews, I. Cionni, E.L. Davin, C. Deser, C. Ehbrecht, P. Friedlingstein, P. Gleckler, K.-D. Gottschaldt, S. Hagemann, M. Juckes, S. Kindermann, J. Krasting, D. Kunert, R. Levine, A. Loew, J. Mäkelä, G. Martin, E. Mason, A. Phillips, S. Read, C. Rio, R. Roehrig, D. Senftleben, A. Sterl, L.H. van Ulft, J. Walton, S. Wang and K.D. Williams, 2015. ESMValTool (v1.0) - a community diagnostic and performance metrics tool for routine 
evaluation of Earth System Models in CMIP. Geosci. Model Dev. Discuss., 8, 7541-7661, doi: 10.5194/gmdd-8-7541-2015

Fan, Y., I.M Held, S.-J. Lin and X.L. Wang, 2013. Ocean Warming Effect on Surface Gravity Wave Climate Change for the End of the 21st Century. J. Clim., 26., 6046-6066, doi: 10.1175/JCLI-D-1200410.1

Fan, Y., S.-J. Lin, S.M. Griffies and M.A. Hemer, .2014. Simulated Global Swell and Wind Sea Climate and Their responses to Anthropogenic Climate Change at the End of the 21st Century. J. Clim., 27, 3516-3536, doi: 10.1175/JCLI-D-13-00198.1

Fasullo, J.T., and K.E. Trenberth, 2008. The annual cycle of the energy budget. Part II: Meridional structures and poleward transports. J.Clim., 21, 2313-2325, doi: 10.1175/2007JCLI1936.1

Flato, G., J. Marotzke, B. Abiodun, P. Braconnot, S.C. Chou, W. Collins, P. Cox, F. Driouech, S. Emori, V. Eyring, C. Forest, P. Gleckler, E. Guilyardi, C. Jakob, V. Kattsov, C. Reason and M. Rummukainen, 2013. Evaluation of Climate Models. In: Climate Change 2013: The Physical Science Basis.

Contribution of Working Group I to the Fifth Assessment Report of the Intergovernmental Panel on Climate Change [Stocker, T.F., D. Qin, G.-K. Plattner, M. Tignor, S.K. Allen, J. Boschung, A. Nauels, Y. Xia, V. Bex and P.M. Midgley (eds.)]. Cambridge University Press, Cambridge, United Kingdom and New York, NY, USA.

Flemming, J., V. Huijnen, J. Arteta, P. Bechtold, A. Beljaars, A.-M. Blechschmidt, M. Diamantakis, R.J. Engelen, A. Gaudel, A. Inness, L. Jones, B. Josse, E. Katragkou, V. Marecal, V.-H. Peuch, A. Richter, M.G. Schultz, O. Stein and A. Tsikerdekis, 2015. Tropospheric Chemistry in the Integrated Forecasting System of ECMWF. Geosci. Model Dev, 8, 975-1003, doi: 10.5194/gmd-8-975-2015

Foley J.A., N. Ramankutty, K.A. Brauman, E.S. Cassidy, J.S. Gerber, M. Johnston, N.D. Mueller, C. O'Connell, D.K. Ray, P.C. West, C. Balzer, E.M. Bennett, S.R. Carpenter, J. Hill, C. Monfreda, S. Polasky, J. Rockström, J. Sheehan, S. Siebert, D. Tilman and D.P.M. Zaks, 2011. Solutions for a cultivated planet. Nature, 478, 337-342, doi: 10.1038/nature10452

Fontana, C., P. Brasseur and J.M. Brankart, 2013. Toward a multivariate reanalysis of the North Atlantic ocean biogeochemistry during 1998-2006 based on the assimilation of SeaWiFS chlorophyll data. Ocean Science, 9, 37-56, doi: 10.5194/os-9-37-2013

Ford, D.A., K.P. Edwards, D. Lea, R.M. Barciela, M.J. Martin and J. Demaria, 2012. Assimilating GlobColour ocean colour data into a pre-operational physical-biogeochemical model. Ocean Sci., 8, 751-771, doi: 10.5194/os-8-751-2012

Fortems-Cheiney, A., F. Chevallier, I. Pison, P. Bousquet, S. Szopa, M. N. Deeter and C. Clerbaux 2011. Ten years of $\mathrm{CO}$ emissions as seen from Measurements of Pollution in the Troposphere (MOPITT). J. Geophys. Res., 116, D05304, doi: 10.1029/2010JD014416

Frankenberg, C., A. Butz and G. C. Toon, 2011. Disentangling chlorophyll fluorescence from atmospheric scattering effects in $\mathrm{O} 2 \mathrm{~A}$-band spectra of reflected sun-light. Geophys. Res. Lett., 38, L03801, doi: 10.1029/2010GL045896 
Fu, L.-L. and B.J. Haines, 2013. The challenges in long-term altimetry calibration for addressing the problem of global sea level change. Adv. Space Res., 51(8), 1284-1300, doi:

10.1016/j.asr.2012.06.005

Fujii, Y., T. Nakaegawa, S. Matsumoto, T. Yasuda, G. Yamanaka and M. Kamachi, 2009. Coupled Climate Simulation by Constraining Ocean Fields in a Coupled Model with Ocean Data. J. Climate, 22, 5541-5557, doi: 10.1175/2009JCLI2814.1

Garcia Leal, J.A., T. Estrela, A. Fidalgo, O. Gabaldo, M. Gonzalez Robles, E. Herrera Daza, S. Khodayar and E. Lopez-Baeza, 2013. Estimation of Surface Runoff in the Jucar River Basin from Rainfall Data and SMOS Soil Moisture. Geophysical Research Abstracts Vol. 15, EGU2013-14259, 2013 EGU General Assembly 2013

Gates, W.L., 1979. The physical basis of climate. Proceedings of the World Climate Conference. WMO, Geneva, 112-131

Gates, W. L., 1992. AMIP: The Atmospheric Model Intercomparison Project. Bull. Amer. Meteor. Soc., 73, 1962-1970

GCOS, 2010. Implementation plan for the global observing system for climate in support of the UNFCCC (2010 Update). Available from www.wmo.int/pages/prog/gcos/ as GCOS Report no. 138

GCOS, 2011. Systematic observation requirements for satellite-based data products for climate. Available from www.wmo.int/pages/prog/gcos/ as GCOS Report no. 154

GCOS/GOOS/WCRP, 2014. Report of the Tropical Pacific Observing System 2020 (TPOS 2020) Workshop. Available from www.wmo.int/pages/prog/gcos/ as GCOS Report no. 184

GCOS, 2015. Status of the Global Observing System for Climate. Available from www.wmo.int/pages/prog/gcos/ as GCOS Report no.195

GEO, 2010. Task US-09-01a: Critical Earth Observation Priorities. Available from sbageotask.larc.nasa.gov

Gettelman, A., H. Morrison, S. Santos, P.A. Bogenschutz and P.M. Caldwell, 2015. Advanced twomoment bulk microphysics for global models. Part II: Global model solutions and aerosol-cloud interactions. J. Clim., 28, 1288-1307, doi: 10.1175/JCLI-D-14-00103.1

Gillett, N.P., V.K. Arora, D. Matthews, P.A. Stott and M.R. Allen, 2013. Constraining the ratio of global warming to cumulative $\mathrm{CO}_{2}$ emissions using CMIP5 simulations. J. Clim., 26, 6844-6858, doi: 10.1175/JCLI-D-12-00476.1

Gleckler, P. J., K. E. Taylor and C. Doutriaux 2008. Performance metrics for climate models, J. Geophys. Res., 113, D06104, doi: 10.1029/2007JD008972.

Gobron, N., and M.M. Verstraete, 2009. FAPAR Fraction of Absorbed Photosynthetically Active Radiation. Essential Climate Variables, T10 Assessment of the status of the development of the standards for the Terrestrial Essential Climate Variables. GTOS 65. Available from www.fao.org/gtos/topcecv.html 
Golaz, J.-C., L.W. Horowitz and H. Levy II, 2013. Cloud tuning in a coupled climate model: impact on 20th century warming. Geophysical Research Letters, 40(10), doi: 10.1002/grl.50232

Gray ,L.J., J. Beer, M. Geller, J.D. Haigh, M. Lockwood, K. Matthes, U. Cubasch, D. Fleitmann, G. Harrison, L. Hood, J. Luterbacher, G.A. Meehl, D. Shindell, B. van Geel and W. White. 2010. Solar influences on climate. Rev. Geophys., 48, RG4001, doi: 10.1029/2009RG000282

Haiden, T., M.J. Rodwell, D.S. Richardson, A. Okagaki, T. Robinson and T. Hewson, 2012. Intercomparison of Global Model Precipitation Forecast Skill in 2010/11 Using the SEEPS Score. Mon. Wea. Rev., 140, 2720-2733, doi: 10.1175/MWR-D-11-00301.1

Haiden, T., M. Janousek, P. Bauer, J. Bidlot, L. Ferranti, T. Hewson, F. Prates, D.S. Richardson and F. Vitart, 2014. Evaluation of ECMWF forecasts, including 2013-2014 upgrades. ECMWF Tech. Memo., 742, 61pp. Available from www.ecmwf.int

Hall, A., 2014. Projecting regional climate change. Science 346, 1461-1462, doi: 10.1126/science.aaa0629

Hawkins, E., and R. Sutton, 2009. The potential to narrow uncertainty in regional climate predictions. Bull. Amer. Meteor. Soc., 90, 1095-1107, doi: 10.1175/2009BAMS2607.1

Hegglin, M.I., D.A. Plummer, T.G. Shepherd, J.F. Scinocca, J. Anderson, L. Froidevaux, B. Funke, D. Hurst, A. Rozanov, J. Urban, T. von Clarmann, K.A. Walker, H.J. Wang, S. Tegtmeier and K. Weigel, 2014. Vertical structure of stratospheric water vapour trends derived from merged satellite data. Nature Geosci., 7, 768-776, doi: 10.1038/ngeo2236

Heimbach, P., G.C. Johnson, B. Sloyan, T. Tanhua, R. Wanninkhof, A. Boetius, L. Levin and M. Sibuet, 2014. Deep ocean observing strategy. Global Ocean Observing System project report. 59pp. Available from www.ioc-goos.org

Hersbach H., C. Peubey, A. Simmons, P. Poli, P. Berrisford and D. Dee, 2015. ERA-20CM: a twentieth century atmospheric model ensemble. Q.J.R. Meteorol. Soc., 141, 2350-2375, doi: 10.1002/qj.2528

Hollingsworth, A., R.J. Engelen, C. Textor, A. Benedetti, O. Boucher, F. Chevallier, A. Dethof, H. Elbern, H. Eskes, J. Flemming, C. Granier, J.W. Kaiser, J.-J. Morcrette, P. Rayner, V.-H. Peuch, L. Rouil, M.G. Schultz, A.J. Simmons and the GEMS Consortium 2008. Toward a monitoring and forecasting system for atmospheric composition: The Gems Project. Bull. Amer. Meteor. Soc., 89, 1147-1164, doi: 10.1175/2008BAMS2355.1

Hollmann, R., C.J. Merchant, R. Saunders, C. Downy, M. Buchwitz, A. Cazenave, E. Chuvieco, P. Defourny, G. de Leeuw, R. Forsberg, T. Holzer-Popp, F. Paul, S. Sandven, S. Sathyendranath, M. van Roozendael and W. Wagner, 2013. The ESA Climate Change Initiative: satellite data records for essential climate variables. Bull. Amer. Meteor. Soc., 94, 1541-1552, doi: 10.1175/BAMS-D-1100254.1

Hoskins BJ, 2013. The potential for skill across the range of the seamless weather-climate prediction problem: a stimulus for our science. Q. J. R. Meteorol. Soc. 139, 573-584, doi: 10.1002/qj.1991 
Houghton, J., J. Townshend, K. Dawson, P. Mason, J. Zillman and A. Simmons, 2012. The GCOS at 20 years: the origin, achievement and future development of the Global Climate Observing System. Weather, 67, 227-235, doi: 10.1002/wea.1964

Houweling, S., M. Krol, P. Bergamaschi, C. Frankenberg, E.J. Dlugokencky, I. Morino, J. Notholt, V. Sherlock, D. Wunch, V. Beck, C. Gerbig, H. Chen, E.A. Kort, T. Röckmann and I. Aben, 2014. A multiyear methane inversion using SCIAMACHY, accounting for systematic errors using TCCON measurements. Atmos. Chem. Phys., 14, 3991-4012, doi: 10.5194/acp-14-3991-2014

Huneeus, N., O. Boucher and F. Chevallier, 2013. Atmospheric inversion of SO2 and primary aerosol emissions for the year 2010. Atmos. Chem. Phys., 13, 6555-6573, doi: 10.5194/acp-13-6555-2013

Hurrell, J., G.A. Meehl, D. Bader, T.L. Delworth, B. Kirtman and B. Wielicki, 2009. A Unified Modeling Approach to Climate System Prediction. Bull. Amer. Meteor. Soc., 90, 1819-1832, doi: 10.1175/2009BAMS2752.1

Illingworth, S., G. Allen, C. Percival, P. Hollingsworth, M. Gallagher, H. Ricketts, H. Hayes, P. Ładosz, D. Crawley and G. Roberts, 2014. Measurement of boundary layer ozone concentrations on-board a Skywalker unmanned aerial vehicle. Atmosph. Sci. Lett., 15, 252-258, doi: 10.1002/asl2.496

Ines, A.V.M., N.N. Das, J.W. Hansen and E.G. Njoku, 2013. Assimilation of remotely sensed soil moisture and vegetation with a crop simulation model for maize yield prediction. Remote Sens. Environ., 138, 149-164, doi: 10.1016/j.rse.2013.07.018

Inness, A., F. Baier, A. Benedetti, I. Bouarar, S. Chabrillat, H. Clark, C. Clerbaux, P. Coheur, R.J. Engelen, Q. Errera, J. Flemming, M. George, C. Granier, J. Hadji-Lazaro, V. Huijnen, D. Hurtmans, L. Jones, J.W. Kaiser, J. Kapsomenakis, K. Lefever, J. Leitão, M. Razinger, A. Richter, M.G. Schultz, A.J. Simmons, M. Suttie, O. Stein, J.-N. Thépaut, V. Thouret, M. Vrekoussis, C. Zerefos and the MACC team, 2013. The MACC reanalysis: an 8 yr data set of atmospheric composition. Atmos. Chem. Phys., 13, 4073-4109, doi: 10.5194/acp-13-4073-2013

IOC/GOOS/JCOMM, 2012. Global Sea-Level Observing System (GLOSS) Implementation Plan - 2012. UNESCO/IOC, 41pp. (IOC Technical Series No.100) (English)

IPCC, 2013. Summary for Policymakers. In: Climate Change 2013: The Physical Science Basis. Contribution of Working Group I to the Fifth Assessment Report of the Intergovernmental Panel on Climate Change [Stocker,T.F., D. Qin, G.-K. Plattner, M. Tignor, S.K. Allen, J. Boschung, A. Nauels, Y. Xia, V. Bex and P.M. Midgley (eds.)]. Cambridge University Press, Cambridge, United Kingdom and New York, NY, USA

Jakob, C., 2010. Accelerating progress in global atmospheric model development through improved parameterizations. Challenges, opportunities, and strategies. Bull. Amer. Meteor.Soc., 91, 869-875, doi: 10.1175/2009BAMS2898.1

Jakob, C., 2014. Going back to basics. Nature Clim. Change, 4, 1042-1045, doi: 10.138/nclimate2445 Janssen, P.A.E.M., 2004. The interaction of ocean waves and wind, Cambridge University Press, $300 \mathrm{pp}$. 
Jia, L., X. Yang, G.A. Vecchi, R.G. Gudgel, T.L Delworth, A. Rosati, W.F. Stern, A.T. Wittenberg, L. Krishnamurthy, S. Zhang, R. Msadek, S. Kapnick, S. Underwood, F. Zeng, W.G. Anderson, V. Balaji and K. Dixon, 2014. Improved seasonal prediction of temperature and precipitation over land in a highresolution GFDL climate model. J. Clim., 28, 2044-2062, doi: 10.1175/JCLI-D-14-00112.1

Jiang, J., Z. Xiao, J. Wang and J. Song, 2014. Sequential method with incremental analysis update to retrieve Leaf Area Index from time series MODIS reflectance data. Remote Sens., 6, 9194-9212, doi: $10.3390 /$ rs6109194

Joiner, J., Y. Yoshida, A.P. Vasilkov, Y. Yoshida, L.A. Corp and E.M. Middleton, 2011. First observations of global and seasonal terrestrial chlorophyll fluorescence from space. Biogeosciences, 8, 637-651, doi: 10.5194/bg-8-637-2011

Jung, M., M. Reichstein, P. Ciais, S.I. Seneviratne, J. Sheffield, M.L. Goulden, G. Bonan, A. Cescatti, J. Chen, R. de Jeu, A.J. Dolman, W. Eugster, D. Gerten, D. Gianelle, N. Gobron, J. Heinke, J. Kimball, B.E. Law, L. Montagnani, Q. Mu, B. Mueller, K. Oleson, D. Papale, A.D. Richardson, O. Roupsard, S. Running, E. Tomelleri, N. Viovy, U. Weber, C. Williams, E. Wood, S. Zaehle and K. Zhang, 2010. Recent decline in the global land evapotranspiration trend due to limited moisture supply. Nature, 467, 951-954, doi: 10.1038/nature09396

Jung, T., M.J. Miller, T.N. Palmer, P. Towers, N. Wedi, D. Achuthavarier, J.M. Adams, E.L. Altshuler, B.A. Cash, J.L. Kinter III, L. Marx, C. Stan and K.I. Hodges, 2012. High-Resolution Global Climate Simulations with the ECMWF Model in Project Athena: Experimental Design, Model Climate, and Seasonal Forecast Skill. J. Climate, 25, 3155-3172, doi: 10.1175/JCLI-D-11-00265.1

Kaiser, J. W., A. Heil, M.O. Andreae, A. Benedetti, N. Chubarova, L. Jones, J.-J. Morcrette, M. Razinger, M.G. Schultz, M. Suttie and G.R. van der Werf, 2012. Biomass burning emissions estimated with a global fire assimilation system based on observed fire radiative power. Biogeosciences, 9 , 527-554, doi: 10.5194/bg-9-527-2012

Kaiser, J.W., N. Andela, J. Atherton, M. de Jong, A. Heil, R. Paugam, S. Remy, M.G. Schultz, G.R. van der Werf, T.T. van Leeuwen and M.J. Wooster, 2014. Recommended Fire Emission Service enhancements. ECMWF Tech. Memo., 724, 82pp. Available from www.ecmwf.int

Kaminski, T., W. Knorr, G. Schürmann, M. Scholze, P.J. Rayner, S. Zaehle, S. Blessing, W. Dorigo, V. Gayler, R. Giering, N. Gobron, J.P. Grant, M. Heimann, A. Hooker-Stroud, S.- Houweling, T. Kato, J. Kattge, D. Kelley, S. Kemp, E.N. Koffi, C. Köstler, P.-P. Mathieu, B. Pinty, C.H. Reick, C. Rödenbeck, R. Schnur, K. Scipal, C. Sebald, T. Stacke, A. Terwisscha van Scheltinga, M. Vossbeck, H. Widmann and T. Ziehn, 2013. The BETHY/JSBACH Carbon Cycle Data Assimilation System: experiences and challenges. J. Geophys. Res. Biogeosci., 118, 1414-1426, doi: 10.1002/jgrg.20118

Kay, J. E., V. Yettella, B. Medeiros, C. Hannay and P. Caldwell, 2015. Global climate impacts of fixing the Southern Ocean shortwave radiation bias in the Community Earth System Model. J. Clim., submitted. Available from www.cgd.ucar.edu/staff/jenkay/papers/Kay_JClim_SouthernOceanASR_submittedMay18,2015.pdf 
Keeley, S.P.E., R.T. Sutton and L.C. Shaffrey, 2012. The impact of North Atlantic sea surface temperature errors on the simulation of North Atlantic European region climate. Q.J.R. Meteorol. Soc., 138, 1774-1783, doi: 10.1002/qj.1912

Kellogg, W.W., 1977. Effects of human activities on global climate. WMO Technical Note No. 156. $47 \mathrm{pp}$.

Kerr, Y.H., P. Waldteufel, P. Richaume, J.-P. Wigneron, P. Ferrazzoli, A. Mahmoodi, A. Al Bitar, F. Cabot, C. Gruhier, S.E. Juglea, D. Leroux, A. Mialon and S.Delwart, 2012. The SMOS Soil Moisture Retrieval Algorithm, IEEE Transactions on Geoscience and Remote Sensing. 50, 1384-1403, doi: 10.1109/TGRS.2012.2184548

Kim, Y., J.S. Kimball, K. Zhang and K.C. McDonald, 2012. Satellite detection of increasing Northern Hemisphere non-frozen seasons from 1979 to 2008. Implications for regional vegetation growth. Remote Sens. Environ., 121, 472-487, doi: 10.1016/j.rse.2012.02.014

Kirschke, S., P. Bousquet, P. Ciais, M. Saunois, J.G. Canadell, E.J. Dlugokencky, P. Bergamaschi, D. Bergmann, D.R. Blake, L. Bruhwiler, P. Cameron-Smith, S. Castaldi, F. Chevallier, L. Feng, A. Fraser, M. Heimann, E.L. Hodson, S. Houweling, B. Josse, P.J. Fraser, P.B. Krummel, J.-F. Lamarque, R.L. Langenfelds, C. Le Quéré, V. Naik, S. O'Doherty, Paul I. Palmer, I. Pison, D. Plummer, B. Poulter, R.G. Prinn, M. Rigby, B. Ringeval, M. Santini, M. Schmidt, D.T. Shindell, I.J. Simpson, R. Spahni, L.P. Steele, S.A. Strode, K. Sudo, S. Szopa, G. R. van der Werf, A. Voulgarakis, M. van Weele, R.F. Weiss, J.E. Williams and G. Zeng, 2013. Three decades of global methane sources and sinks. Nature Geosci., 6, 813-823, doi: 10.1038/ngeo1955

Kirtman, B., S.B. Power, J.A. Adedoyin, G.J. Boer, R. Bojariu, I. Camilloni, F.J. Doblas-Reyes, A.M. Fiore, M. Kimoto, G.A. Meehl, M. Prather, A. Sarr, C. Schär, R. Sutton, G.J. van Oldenborgh, G. Vecchi and H.J. Wang, 2013. Near-term Climate Change: Projections and Predictability. In: Climate Change 2013: The Physical Science Basis. Contribution of Working Group I to the Fifth Assessment Report of the Intergovernmental Panel on Climate Change [Stocker, T.F., D. Qin, G.-K. Plattner, M. Tignor, S.K. Allen, J. Boschung, A. Nauels, Y. Xia, V. Bex and P.M. Midgley (eds.)]. Cambridge University Press, Cambridge, United Kingdom and New York, NY, USA.

Kobayashi, S., Y. Ota, Y. Harada, A. Ebita, M. Moriya, H. Onoda, K. Onogi, H. Kamahori, C. Kobayashi, H. Endo, K. Miyaoka and K. Takahashi, 2015. The JRA-55 Reanalysis: General specifications and basic characteristics. J. Meteorol. Soc. Japan, 93, 5-48, doi: 10.2151/jmsj.2015-001

Koh, L. P., and S.A. Wich, 2012. Dawn of drone ecology: low-cost autonomous aerial vehicles for conservation. Tropical Conservation Science, 5(2),121-132

Komurcu, M., T. Storelvmo, I. Tan, U. Lohmann, Y. Yun, J. E. Penner, Y. Wang, X. Liu and T. Takemura, 2014. Intercomparison of the cloud water phase among global climate models. J. Geophys. Res.

Atmos., 119, 3372-3400, doi: 10.1002/2013JD021119

Koven, C.D., W J. Riley and A. Stern, 2013. Analysis of permafrost thermal dynamics and response to climate change in the CMIP5 Earth system models. J. Clim., 26, 1877-1900, doi: 10.1175/JCLI-D-1200228.1 
Kristiansen, N.I., A. Stohl, A.J. Prata, N. Bukowiecki, H. Dacre, S. Eckhardt, S. Henne, M.C. Hort, B.T. Johnson, F. Marenco, B. Neininger, O. Reitebuch, P. Seibert, D.J. Thomson, H.N. Webster and B. Weinzierl , 2012. Performance assessment of a volcanic ash transport model mini-ensemble used for inverse modeling of the 2010 Eyjafjallajökull eruption. J. Geophys. Res., 117, D00U11, doi: 10.1029/2011JD016844

Kulmala, M., V.M. Kerminen, T. Anttila, A. Laaksonen and C. O'Dowd, 2004. Organic aerosol formation via sulphate cluster activation. J. Geophys. Res., 109, D04205, doi: 10.1029/2003JD003961, 2004

Kumar, A., M. Chen, L. Zhang, W. Wang, Y. Xue, C. Wen, L. Marx and B. Huang, 2012. An analysis of the nonstationarity in the bias of sea surface temperature Forecasts for the NCEP Climate Forecast System (CFS) Version 2. Mon. Wea. Rev., 140, 3003-3016, doi: 10.1175/MWR-D-11-00335.1

Kuppel, S., P. Peylin, F. Chevallier, C. Bacour, F. Maignan and A.D. Richardson, 2012. Constraining a global ecosystem model with multi-site eddy-covariance data. Biogeosciences, 9, 3757-3776, doi: 10.5194/bg-9-3757-2012

Kurtén, T., V. Loukonen, H. Vehkamäki and M. Kulmala, 2008. Amines are likely to enhance neutral and ion-induced sulfuric acid-water nucleation in the atmosphere more effectively than ammonia. Atmos. Chem. Phys., 8, 4095-4103, doi: 10.5194/acp-8-4095-2008

Laloyaux, P., M. Balmaseda, D. Dee, K.Mogensen and P. Janssen, 2015. A coupled data assimilation system for climate reanalysis. Q. J. R. Meteorol. Soc., doi: 10.1002/qj.2629

Lamarque, J.-F., D.T. Shindell, B. Josse, P.J. Young, I. Cionni, V. Eyring, D. Bergmann, P. CameronSmith, W.J. Collins, R. Doherty, S. Dalsoren, G. Faluvegi, G. Folberth, S.J. Ghan, L.W. Horowitz, Y.H. Lee,I.A. MacKenzie, T. Nagashima, V. Naik, D. Plummer, M. Righi, S.T. Rumbold, M. Schulz, R.B. Skeie, D.S. Stevenson, S. Strode, K. Sudo, S. Szopa, A. Voulgarakis and G. Zeng, 2013. The Atmospheric Chemistry and Climate Model Intercomparison Project (ACCMIP): overview and description of models, simulations and climate diagnostics. Geosci. Model Dev., 6, 179-206, doi: 10.5194/gmd-6$179-2013$

Lange, A., and E. Hellsten, 1984. Results of theWMO/CASNWPData Study and Intercomparison Project for forecasts for the northern hemisphere. Short and Medium Range Weather Prediction Research Publication Series, 7, WMO, Geneva, Switzerland

Lattanzio, A., J. Schulz, J. Matthews, A. Okuyama, B. Theodore, J.J. Bates, K.R. Knapp, Y. Kosaka and L. Schüller, 2013. Land Surface Albedo from Geostationary Satelites: A Multiagency Collaboration within SCOPE-CM. Bull. Amer. Meteor. Soc., 94, 205-214, doi: 10.1175/BAMS-D-11-00230.1

Lawford, R. (Ed.), 2014. The GEOSS Water Strategy: From Observations to Decisions. Available through www.earthobservations.org, 255pp.

Le Quéré, C., G.P. Peters, R.J. Andres, R. M. Andrew, T.A. Boden, P Ciais, P. Friedlingstein, R.A. Houghton, G. Marland, R. Moriarty, S. Sitch, P. Tans, A. Arneth, A. Arvanitis, D.C.E. Bakker, L. Bopp, J.G. Canadell, L.P. Chini, S.C. Doney, A. Harper, I. Harris, J.I. House, A.K. Jain, S.D. Jones, E. Kato, R.F. Keeling, K. Klein Goldewijk, A. Körtzinger, C. Koven, N. Lefèvre, F. Maignan, A. Omar, T. Ono, G.-H. 
Park, B. Pfeil, B. Poulter, M.R. Raupach, P. Regnier, C. Rödenbeck, S. Saito, J. Schwinger, J. Segschneider, B.D. Stocker, T. Takahashi, B. Tilbrook, S. van Heuven, N. Viovy, R. Wanninkhof, A. Wiltshire and S. Zaehle, 2014. Global carbon budget 2013. Earth Syst. Sci. Data, 6, 235-263, doi: 10.5194/essd-6-235-2014

Liao, M., P. Zhang, G.L. Yang, Y.M. Bi, Y. Liu, W.H. Bai, X.G. Meng, Q.F. Du and Y.Q. Sun, 2015. Preliminary validation of refractivity from a new radio occultation sounder GNOS/FY-3C. Atmos. Meas. Tech. Discuss., 8, 9009-9044, doi:10.5194/amtd-8-9009-2015

Lin, Y., L. J. Donner, J. Petch, P. Bechtold, J. Boyle, S. A. Klein, T. Komori, K. Wapler, M. Willett, X. Xie, M. Zhao, S. Xie, S.A. McFarlane and C. Schumacher, 2012. TWP-ICE global atmospheric model intercomparison: convection responsiveness and resolution impact. J. Geophys. Res., 117(D09111), doi: 10.1029/2011JD017018

Lin, G., S. Sillman, J.E. Penner and A. Ito, 2014. Global modeling of SOA: The use of different mechanisms for aqueous-phase formation. Atmos. Chem. Phys., 14, 5451-5475, doi: 10.5194/acp14-5451-2014

Lindstrom, E., J. Gunn, A. Fischer, A. McCurdy, L. K. Glover and task-team members, 2012. A Framework for Ocean Observing. Report of the Task Team for an Integrated Framework for Sustained Ocean Observing, UNESCO 2012, IOC/INF-1284 rev., doi: 10.5270/OceanObs09-FOO

Lionello, P., H. Günther and P. A. E. M. Janssen, 1992. Assimilation of altimeter data in a global thirdgeneration wave model. J. Geophys. Res., 97(C9), 14453-14474, doi: 10.1029/92JC01055

Loew A., P, M. van Bodegom, J.-L. Widlowski, J. Otto, T. Quaife, B. Pinty and T. Raddatz, 2014. Do we (need to) care about canopy radiation schemes in DGVMs? Caveats and potential impacts. Biogeosciences, 11, 1873-1897, doi: 10.5194/bg-11-1873-2014

Lopez-Baeza, E., J.A. Garcia Leal, T. Estrela, R. Fernandez, A. Fidalgo, O. Gabaldo, M. Gonzalez Robles, E. Herrera Daza, S. Khodayar, 2014. Estimation of surface runoff in the Jucar River Basin from raingauge precipitation measurements and soil moisture products from SMOS. Ch 12 in Applications of Supporting Systems to Integrated Planning and Management Decision in Hydrological Basins. Marcombo Ediciones Tecnicas, pg. 123-128, ISBN 978-84-267-2161-7

Lorenz, E.N., 1975. Climatic predictability. In The Physical Basis of Climate Modelling, Global Atmospheric Research Programme (GARP) Publication Series, 16, 132-136

Lorenz, E.N., 1995: Predictability - a partly solved problem. Proceedings of 1995 ECMWF Seminar on predictability. Available from www.ecmwf.int. Reproduced in Palmer and Hagedorn, 2006.

Predictability of Weather and Climate, Cambridge University Press, 702pp

Lott, F.C., P.A. Stott, D.M. Mitchell, N. Christidis, N.P. Gillett, L. Haimberger, J. Perlwitz and P.W. Thorne, 2013. Models versus radiosondes in the free atmosphere: A new detection and attribution analysis of temperature. J. Geophys. Res. Atmos., 118, 2609-2619, doi: 10.1002/jgrd.50255

Mahoney, W.P. III, and J.M. O'Sullivan, 2013. Realizing the Potential of Vehicle-Based Observations. Bull. Amer. Meteor. Soc., 94, 1007-1018, doi: 10.1175/BAMS-D-12-00044.1 
Maksyutov, S., H. Takagi, V.K. Valsala, M. Saito, T. Oda, T. Saeki, D.A. Belikov, R. Saito, A. Ito, Y. Yoshida, I. Morino, O. Uchino, R.J. Andres and T. Yokota, 2013. Regional $\mathrm{CO}_{2}$ flux estimates for 2009 2010 based on GOSAT and ground-based CO2 observations. Atmos. Chem. Phys., 13, 9351-9373, doi: 10.5194/acp-13-9351-2013

Manabe, S., and R. T. Wetherald, 1975. The effects of doubling the $\mathrm{CO}_{2}$ concentration on the climate of a General Circulation Model. J. Atmos. Sci., 32, 3-15, doi: 10.1175/1520-

0469(1975)032<0003:TEODTC>2.0.CO;2

Manabe, S., K. Bryan and M.J. Spelman, 1975. A global ocean-atmosphere climate model. Part I. The atmospheric circulation. J. Phys. Oceanogr., 5, 3-29, doi: 10.1175/1520-

0485(1975)005<0003:AGOACM>2.0.CO;2

Mao, J., F. Paulot, D.J. Jacob, R.C. Cohen, J.D. Crounse, P.O. Wennberg, C.A. Keller, R. Hudman, M.P. Barkley and L.W. Horowitz, 2013. Ozone and organic nitrates over the eastern United States: sensitivity to isoprene chemistry. Journal of Geophysical Research, 118, 11256-11268, doi: 10.1002/jgrd.50817

Martín-Neira, M., J. Hatton, M. Kern, J. Rosello, S. D'Addio and N. Floury, 2014. System Requirements for the GEROS-ISS Payload. International Geoscience and Remote Sensing Symposium (IGARSS 2014) / 35th Canadian Symposium on Remote Sensing (35th CSRS), Quebec, Canada, 13-18 July 2014

Mass, C.F., and L.E. Madaus, 2014. Surface Pressure Observations from Smartphones: A Potential Revolution for High-Resolution Weather Prediction? Bull. Amer. Meteor. Soc., 95, 1343-1349, doi: 10.1175/BAMS-D-13-00188.1

Massart, S., A. Agusti-Panareda, I. Aben, A. Butz, F. Chevallier, C. Crevoisier, R. Engelen, C. Frankenberg and O. Hasekamp, 2014. Assimilation of atmospheric methane products into the MACCII system: from SCIAMACHY to TANSO and IASI, Atmos. Chem. Phys., 14, 6139-6158, doi: 10.5194/acp-14-6139-2014

Masson-Delmotte, V., M. Schulz, A. Abe-Ouchi, J. Beer, A. Ganopolski, J.F. González Rouco, E. Jansen, K. Lambeck, J. Luterbacher, T. Naish, T. Osborn, B. Otto-Bliesner, T. Quinn, R. Ramesh, M. Rojas, X. Shao and A. Timmermann, 2013. Information from Paleoclimate Archives. In: Climate Change 2013: The Physical Science Basis. Contribution of Working Group I to the Fifth Assessment Report of the Intergovernmental Panel on Climate Change [Stocker, T.F., D. Qin, G.-K. Plattner, M. Tignor, S.K. Allen, J. Boschung, A. Nauels, Y. Xia, V. Bex and P.M. Midgley (eds.)]. Cambridge University Press, Cambridge, United Kingdom and New York, NY, USA.

McMillan, H.K., E.Ö. Hreinsson, M.P. Clark, S.K. Singh, C. Zammit and M.J. Uddstrom, 2013. Operational hydrological data assimilation with the recursive ensemble Kalman filter. Hydrol. Earth Syst. Sci., 17, 21-38, doi: 10.5194/hess-17-21-2013

Meehl, G.A., L. Goddard, G. Boer, R. Burgman, G. Branstator, C. Cassou, S. Corti, G. Danabasoglu, F. Doblas-Reyes, E. Hawkins, A. Karspeck, M. Kimoto, A. Kumar, D. Matei, J. Mignot, R. Msadek, A. Navarra, H. Pohlmann, M. Rienecker, A. Rosati, E. Schneider, D. Smith, R. Sutton, H. Teng, G. J. van Oldenborgh, G. Vecchi and S. Yeager, 2014. Decadal Climate Prediction: An Update from the Trenches. Bull. Amer. Meteor. Soc., 95, 243-267, doi: 10.1175/BAMS-D-12-00241.1 
Meier, W.N., D. Gallaher and G. G. Campbell, 2013. New estimates of Arctic and Antarctic sea ice extent during September 1964 from recovered Nimbus I satellite imagery. The Cryosphere, 7, 699705, doi:10.5194/tc-7-699-2013

Menkes, C.E., M. Lengaigne, J. Vialard, M. Puy, P. Marchesiello, S. Cravatte and G. Cambon, 2014. About the role of Westerly Wind Events in the possible development of an El Niño in 2014. Geophys. Res. Lett., 41, 6476-6483, doi: 10.1002/2014GL061186

Mijling, B., and R.J. van der A, 2012. Using daily satellite observations to estimate emissions of shortlived air pollutants on a mesoscopic scale. J. Geophys. Res., 117, D17302, doi: 10.1029/2012JD017817

Mitchell, D.M., P.W. Thorne, P.A. Stott and L.J. Gray, 2013. Revisiting the controversial issue of tropical tropospheric temperature trends. Geophys. Res. Lett., 40, 2801-2806, doi: 10.1002/grl.50465

Miyazaki, K., H.J. Eskes, K. Sudo and C. Zhang, 2014. Global lightning NOx production estimated by an assimilation of multiple satellite data sets. Atmos. Chem. Phys., 14, 3277-3305, doi: 10.5194/acp-143277-2014

Monge-Sanz, B. M., M.P. Chipperfield, A. Untch, J.-J. Morcrette, A. Rap and A.J. Simmons, 2013. On the uses of a new linear scheme for stratospheric methane in global models: water source, transport tracer and radiative forcing. Atmos. Chem. Phys., 13, 9641-9660, doi: 10.5194/acp-13-9641-2013

Monteil, G., S. Houweling, A. Butz, S. Guerlet, D. Schepers, O. Hasekamp, C. Frankenberg, R. Scheepmaker, I. Aben and T. Röckmann, 2013. Comparison of $\mathrm{CH} 4$ inversions based on 15 months of GOSAT and SCIAMACHY observations. J. Geophys. Res. Atmos., 118, 11,807-11,823, doi: 10.1002/2013JD019760

Muñoz-Sabater, J., 2015. Incorporation of passive microwave brightness temperatures in the ECMWF soil moisture analysis. Remote Sens., 7, 5758-5784, doi: 10.3390/rs70505758

Myhre, G., D. Shindell, F.-M. Bréon, W. Collins, J. Fuglestvedt, J. Huang, D. Koch, J.-F. Lamarque, D. Lee, B. Mendoza, T. Nakajima, A. Robock, G. Stephens, T. Takemura and H. Zhang, 2013.

Anthropogenic and Natural Radiative Forcing. In: Climate Change 2013: The Physical Science Basis. Contribution of Working Group I to the Fifth Assessment Report of the Intergovernmental Panel on Climate Change [Stocker, T.F., D. Qin, G.-K. Plattner, M. Tignor, S.K. Allen, J. Boschung, A. Nauels, Y. Xia, V. Bex and P.M. Midgley (eds.)]. Cambridge University Press, Cambridge, United Kingdom and New York, NY, USA.

Nevison, C.D., M. Manizza, R.F. Keeling, M. Kahru, L. Bopp, J. Dunne, J. Tiputra, T. Ilyina and B.G. Mitchell, 2015. Evaluating the ocean biogeochemical components of Earth system models using atmospheric potential oxygen and ocean color data. Biogeosci., 12, 193-208, doi: 10.5194/bg-12193-2015

Niu, S., Y. Luo, M. C. Dietze, T. F. Keenan, Z. Shi, J. Li and F. S. Chapin III, 2014. The role of data assimilation in predictive ecology. Ecosphere 5(5), 65, doi: 10.1890/ES13-00273.1 
Noetzli, J., H.H. Christiansen, M. Gugliemin, V.E. Romanovsky, N.I. Shiklomanov, S.L. Smith and L. Zhao , 2014. [Global climate] Permafrost thermal state [in "State of the Climate in 2013"]. Bull. Amer. Meteor. Soc., 95 (7), S15-S17.

NRC, 1975. Understanding Climatic Change - A program for action. Report of the US Committee for GARP. National Academy of Sciences, Washington DC, 237pp.

NRC, 1979. Carbon dioxide and climate: A scientific assessment. Report of an Ad Hoc Study Group. National Academy of Sciences, Washington DC, 22pp.

NRC, 2007. Earth Science and Applications from Space: National Imperatives for the Next Decade and Beyond. National Academy of Sciences, Washington DC, 456pp.

Palmer, T.N., and D.L.T. Anderson, 1994. The prospects for seasonal forecasting-A review paper. Q.J.R. Meteorol. Soc., 120, 755-793, doi: 10.1002/qj.49712051802

Palmer, T.N., F.J. Doblas-Reyes, A. Weisheimer and M.J. Rodwell, 2008. Toward Seamless Prediction: Calibration of Climate Change Projections Using Seasonal Forecasts. Bull. Amer. Meteor. Soc., 89, 459-470, doi: 10.1175/BAMS-89-4-459

Pan, Y. D., R.A. Birdsey, J. Fang, R. Houghton, P.E. Kauppi, W.A. Kurz, O.L. Phillips, A. Shvidenko, S.L. Lewis, J.G. Canadell, P. Ciais, R.B. Jackson, S.W. Pacala, A.D. McGuire, S.Piao, A. Rautiainen, S. Sitch and D. Hayes, 2011. A large and persistent carbon sink in the world's forests. Science, 333, 988-993. doi: 10.1126/science.1201609

Panet, I., J. Flury, R. Biancale, T. Gruber, J. Johannessen, M. van den Broeke, T. van Dam, P. Gegout, C. Hughes, G. Ramillien, I. Sasgen, L. Seoane and M. Thomas, 2013. Earth system mass transport mission (e.motion): A concept for future Earth gravity field measurements from space. Surveys in Geophysics, 34 (2), 141-163, doi: 10.1007/s10712-012-9209-8

Pendergrass, A., and D. Hartmann, 2014. The atmospheric energy constraint on global- mean precipitation change. J. Clim., 27, 757-768, doi: JCLI-D-13-00163.1.

Penner, J. E., J. Quaas, T. Storelvmo, T. Takemura, O. Boucher, H. Guo, A. Kirkevåg, J.E. Kristjánsson and $\varnothing$. Seland, 2006. Model intercomparison of indirect aerosol effects. Atmos. Chem. Phys., 6, 3391-3405, doi: 10.5194/acp-6-3391-2006

Pereira, H.M., S. Ferrier, M. Walters, G.N. Geller, R.H.G. Jongman, R.J. Scholes, M.W. Bruford, N. Brummitt, S.H.M. Butchart, A.C. Cardoso, N.C. Coops, E. Dulloo, D.P. Faith, J. Freyhof, R.D. Gregory, C. Heip, R. Höft, G. Hurtt, W. Jetz, D. S. Karp, M.A. McGeoch, D. Obura, Y. Onoda, N. Pettorelli, B. Reyers, R. Sayre, J.P.W. Scharlemann, S.N. Stuart, E. Turak, M. Walpole and M. Wegmann, 2013. Essential Biodiversity Variables. Science, 339, 277-278, doi: 10.1126/science.1229931

Peters, W., A.R. Jacobson, C. Sweeney, A.E. Andrews, T.J. Conway, K. Masarie, J.B. Miller, L.M.P. Bruhwiler, G. Pétron, A.I. Hirsch, D.E.J. Worthy, G.R. van der Werf, J.T. Randerson, P.O. Wennberg, M.C. Krol and P.P. Tans, 2007. An atmospheric perspective on North American carbon dioxide exchange: CarbonTracker. PNAS, 104(48), 18925-18930, doi: 10.1073/pnas.0708986104 
Peylin, P., R.M. Law, K.R. Gurney, F. Chevallier, A.R. Jacobson, T. Maki, Y. Niwa, P.K. Patra, W. Peters, P.J. Rayner, C. Rödenbeck, I.T. van der Laan-Luijkx and X. Zhang, 2013. Global atmospheric carbon budget: results from an ensemble of atmospheric CO2 inversions. Biogeosciences, 10, 6699-6720, doi: 10.5194/bg-10-6699-2013

Pleskachevsky, A., S. Lehner, T. Heege and C. Mott, 2011. Synergy and fusion of optical and synthetic aperture radar satellite data for underwater topography estimation in coastal areas. Ocean Dynamics, 61, 2099-2120, doi: 10.1007/s10236-011-0460-1

Quaas, J., Y. Ming, S. Menon, T. Takemura, M. Wang, J.E. Penner, A. Gettelman, U. Lohmann, N. Bellouin, O. Boucher, A.M. Sayer, G.E. Thomas, A. McComiskey, G. Feingold, C. Hoose, J.E. Kristjánsson, X. Liu, Y. Balkanski, L.J. Donner, P.A. Ginoux, P. Stier, J. Feichter, I. Sednev, S.E. Bauer, D. Koch, R.G. Grainger, A. Kirkevåg, T. Iversen, $\varnothing$. Seland, R. Easter, S.J. Ghan, P.J. Rasch, H. Morrison, J.F. Lamarque, M.J. Iacono, S. Kinne and M. Schulz, 2009. Aerosol indirect effects - general circulation model intercomparison and evaluation with satellite data. Atmos. Chem. Phys., 9, 8697-8717, doi: 10.5194/acp-9-8697-2009

Reager, J.T., B.F. Thomas and J.S. Famiglietti, 2014. River basin flood potential inferred using GRACE gravity observations at several months lead time. Nature Geoscience, 7, 588-592, doi: $10.1038 /$ ngeo2203

Reichle, R.H., R.D. Koster, G.J.M. De Lannoy, B.A. Forman, Q. Liu, S.P.P. Mahanama and A. Touré, 2011. Assessment and Enhancement of MERRA Land Surface Hydrology Estimates. J. Climate, 24, 6322-6338, doi: 10.1175/JCLI-D-10-05033.1

Reuter, M., M. Buchwitz, M. Hilker, J. Heymann, O. Schneising, D. Pillai, H. Bovensmann, J. P. Burrows, H. Bösch, R. Parker, A. Butz, O. Hasekamp, C.W. O’Dell, Y. Yoshida, C. Gerbig, T. Nehrkorn, N. M. Deutscher, T. Warneke1, J. Notholt, F. Hase, R. Kivi, R. Sussmann, T. Machida, H. Matsueda and Y. Sawa, 2014. Satellite-inferred European carbon sink larger than expected. Atmos. Chem. Phys., 14, 13739-13753, doi:10.5194/acp-14-13739-2014

Rhein, M., S.R. Rintoul, S. Aoki, E. Campos, D. Chambers, R.A. Feely, S. Gulev, G.C. Johnson, S.A. Josey, A. Kostianoy, C. Mauritzen, D. Roemmich, L.D. Talley and F. Wang, 2013. Observations: Ocean. In: Climate Change 2013: The Physical Science Basis. Contribution of Working Group I to the Fifth Assessment Report of the Intergovernmental Panel on Climate Change [Stocker, T.F., D. Qin, G.-K. Plattner, M. Tignor, S.K. Allen, J. Boschung, A. Nauels, Y. Xia, V. Bex and P.M. Midgley (eds.)]. Cambridge University Press, Cambridge, United Kingdom and New York, NY, USA.

Ridler, M.-E., H. Madsen, S. Stisen, S. Bircher and R. Fensholt, 2014. Assimilation of SMOS-derived soil moisture in a fully integrated hydrological and soil-vegetation-atmosphere transfer model in Western Denmark. Water Resour. Res., 50, 8962-8981, doi: 10.1002/2014WR015392

Rienecker, M.M., M.J. Suarez, R. Gelaro, R. Todling, J. Bacmeister, E. Liu, M.G. Bosilovich, S.D. Schubert, L. Takacs, G.-J. Kim, S. Bloom, J. Chen, D. Collins, A, Conaty, A. da Silva, W. Gu, J. Joiner, R.D. Koster, R. Lucchesi, A. Molod, T. Owens, S. Pawson, P. Pegion, C.R. Redder, R. Reichle, F.R. Robertson, A.G. Ruddick, M. Sienkiewicz and J. Woollen, 2011. MERRA: NASA's Modern-Era Retrospective Analysis for Research and Applications. J. Climate, 24, 3624-3648, doi: 10.1175/JCLI-D11-00015.1 
Rintoul, S., E. van Wijk, A. Wåhlin, F. Taylor, L. Newman, S. Ackley, O. Boebel, L. Boehme, A. Bowen, M. Dzieciuch, B. Galton-Fenzi, L. Herraiz-Borreguero, H. Kyung Ha, K. Heywood, M. Hindell, D. Holland, S. Jacobs, A. Jenkins, A. Klepikov, C. Lee, S. Lee, J. Liu, R. Massom, M. Mata, W. Munk, A. Naveira Garabato, K. Nicholls, K. Ohshima, A. Orsi, S. Österhus, B. Owens, B. Peña-Molino, S. Piotrowicz, S. Riser, R. Robertson, T. Sato, K. Speer, J. Toole, M. Williams and C. Yoo, 2014. Seeing Below the ice: A strategy for observing the ocean beneath Antarctic sea ice and ice shelves, Report of the Southern Ocean Observing System. 57pp. Available from www.soos.aq/

Rödenbeck, C., D.C.E. Bakker, N. Metzl, A. Olsen, C. Sabine, N. Cassar, F. Reum, R.F. Keeling and M. Heimann, 2014. Interannual sea-air $\mathrm{CO}_{2}$ flux variability from an observation-driven ocean mixedlayer scheme. Biogeosciences, 11, 4599-4613, doi: 10.5194/bg-11-4599-2014

Rodwell, M.J., D.S. Richardson, T.D. Hewson and T. Haiden, 2010. A new equitable score suitable for verifying precipitation in numerical weather prediction. Q.J.R. Meteorol. Soc., 136, 1344-1363, doi: 10.1002/qj.656

Romanovsky, V.E., S.L. Smith, H.H. Christiansen, N.I. Shiklomanov, D.A. Streletskiy, D.S. Drozdov, G.V. Malkova, N.G. Oberman, A.L. Kholodov and S.S. Marchenko, 2014. [The Arctic] Terrestrial permafrost [in "State of the Climate in 2013"]. Bull. Amer. Meteor. Soc., 95 (7), S139-S141.

Rummel, R., W. Yi and C. Stummer, 2011. GOCE gravitational gradiometry. J. Geodesy, 85(11), 777790, doi: 10.1007/s00190-011-0500-0

Saha, S., S. Moorthi, H.-L. Pan, X. Wu, J. Wang, S. Nadiga, P. Tripp, R. Kistler, J. Woollen, D. Behringer, H. Liu, D. Stokes, R. Grumbine, G. Gayno, J. Wang, Y.-T. Hou, H.-Y. Chuang, H.-M.H. Juang, J. Sela, M. Iredell, R. Treadon, D. Kleist, P. Van Delst, D. Keyser, J. Derber, M. Ek, J Meng, H. Wei, R. Yang, S. Lord, H. Van Den Dool, A. Kumar, W. Wang, C. Long, M. Chelliah, Y. Xue, B. Huang, J.-K. Schemm, W. Ebisuzaki, R. Lin, P. Xie, M. Chen, S. Zhou, W. Higgins, C.-Z. Zou, Q. Liu, Y. Chen, Y. Han, L. Cucurull, R.W. Reynolds, G. Rutledge and M. Goldberg, 2010. The NCEP Climate Forecast System Reanalysis. Bull. Amer. Meteor. Soc., 91: 1015-1057, doi: 10.1175/2010BAMS3001.1

Sandwell, D.T., R.D. Müller, W.H.F. Smith, E. Garcia and R. Francis, 2014. New global marine gravity model from CryoSat-2 and Jason-1 reveals buried tectonic structure. Science, 346 (6205), 65-67, doi: $10.1126 /$ science. 1258213

Santer, B.D., C. Bonfils, J.F. Painter, M.D. Zelinka, C. Mears, S. Solomon, G.A. Schmidt, J.C. Fyfe, J.N.S. Cole, L. Nazarenko, K.E. Taylor and F.J. Wentz, 2014. Volcanic contribution to decadal changes in tropospheric temperature. Nature Geosci., 7, 185-189, doi: 10.1038/ngeo2098

von Savigny, C., F. Ernst, A. Rozanov, R. Hommel, K.-U. Eichmann, V. Rozanov, J.P. Burrows and L.W. Thomason, 2015. Improved stratospheric aerosol extinction profiles from SCIAMACHY: validation and sample results. Atmos. Meas. Tech., 8, 5223-5235, doi:10.5194/amt-8-5223-2015

Schaaf, C.B., F. Gao, A.H. Strahler, W. Lucht, X. Li, T. Tsang, N.C. Strugnell, X. Zhang, Y. Jin, J.-P. Muller, P. Lewis, M. Barnsley, P. Hobson, M. Disney, G. Roberts, M. Dunderdale, C. Doll, R.P. d'Entremont, B. Hu, S. Liang, J.L. Privette and D. Roy, 2002. First operational BRDF, albedo nadir reflectance products from MODIS. Remote Sens. Environ., 83, 135-148, doi: 10.1016/S00344257(02)00091-3 
Schneising, O., J.P. Burrows, R.R. Dickerson, M. Buchwitz, M. Reuter, and H. Bovensmann, 2014. Remote sensing of fugitive methane emissions from oil and gas production in North American tight geologic formations. Earth's Future, 2, 548-558, doi: 10.1002/2014EF000265

Schrijver, C.J., K. Kauristie, A.D. Aylward, C.M. Denardini, S.E. Gibson, A. Glover, N. Gopalswamy, M. Grande, M. Hapgood, D. Heynderickx, N. Jakowski, V.V. Kalegaev, G. Lapenta, J.A. Linker, S. Liu, C.H. Mandrini, I.R. Mann, T. Nagatsuma, D. Nandy, T. Obara, T.P. O'Brien, T. Onsager, H.J. Opgenoorth, M. Terkildsen, C.E. Valladares and N. Vilmer, 2015. Understanding space weather to shield society: A global road map for 2015-2025 commissioned by COSPAR and ILWS. Adv. Space Res., 55, 2745-2807, doi: 10.1016/j.asr.2015.02.023

Seidel, D.J., F.H. Berger, F. Immler, M. Sommer, H. Vömel, H.J. Diamond, J. Dykema, D. Goodrich, W. Murray, T. Peterson, D. Sisterson, P. Thorne and J. Wang, 2009. Reference Upper-Air Observations for Climate: Rationale, Progress, and Plans. Bull. Amer. Meteor. Soc., 90, 361-369, doi: 10.1175/2008BAMS2540.1

Shepherd, T.G., D.A. Plummer, J.F. Scinocca, M.I. Hegglin, V.E. Fioletov, M.C. Reader, E. Remsberg, T. von Clarmann and H. J. Wang, 2014. Reconciliation of halogen-induced ozone loss with the totalcolumn ozone record. Nature Geosci, 7, 443-449, doi: 10.1038/ngeo2155

Sherwood, S.C., S. Bony and J.L Dufresne, 2014. Spread in model climate sensitivity traced to atmospheric convective mixing. Nature, 505, 37-42, doi: 10.1038/nature12829

Simmons, A.J., and A. Hollingsworth, 2002. Some aspects of the improvement in skill of numerical weather prediction. Q.J.R. Meteorol. Soc., 128, 647-677, doi: 10.1256/003590002321042135

Simmons, A.J., P. Poli, D.P. Dee, P. Berrisford, H. Hersbach, S. Kobayashi and C. Peubey, 2014. Estimating low-frequency variability and trends in atmospheric temperature using ERA-Interim. Q.J.R. Meteorol. Soc., 140, 329-353, doi: 10.1002/qj.2317

Simmons. A.J., and P. Poli, 2015. Arctic warming in ERA-Interim and other analyses. Q.J.R. Meteorol. Soc., 141, 1147-1162, doi: 10.1002/qj.2422

Skofronick-Jackson, G., W.A. Petersen, A.Y. Hou, E.F. Stocher, J. Kaye and R. Kakar, 2013. GPM Science Implementation Plan. Available from pmm.nasa.gov/resources/documents/GPM

Smith, J.N., K.C. Barsanti, Hans.R. Friedli, M. Ehn, M. Kulmala, D.R. Collins, J.H. Scheckman, B.J. Williams and P.H. McMurry, 2010. Observations of aminium salts in atmospheric nanoparticles and possible climatic implications. PNAS, 107 (15), 6634-6639, doi: 10.1073/pnas.0912127107

Stephens, G.L., and Y. Hu, 2010. Are climate-related changes to the character of global-mean precipitation predictable? Environ. Res. Lett., 5, doi: 10.1088/1748-9326/5/2/025209

Stephens, G.L., T. L'Ecuyer, R. Forbes, A. Gettlemen, J.-C. Golaz, A. Bodas-Salcedo, K. Suzuki, P. Gabriel and J. Haynes, 2010. Dreary state of precipitation in global models. J. Geophys. Res., 115, D24211, doi: 10.1029/2010JD014532 
Stockdale, T.N., D.L.T. Anderson, M.A. Balmaseda, F. Doblas-Reyes, L. Ferranti, K. Mogensen, T.N. Palmer, F. Molteni and F. Vitart, 2011. ECMWF seasonal forecast system 3 and its prediction of sea surface temperature. Climate Dynamics, 37, 455-471, doi: 10.1007/s00382-010-0947-3

Stocker, T.F., D. Qin, G.-K. Plattner, L.V. Alexander, S.K. Allen, N.L. Bindoff, F.-M. Bréon, J.A. Church, U. Cubasch, S. Emori, P. Forster, P. Friedlingstein, N. Gillett, J.M. Gregory, D.L. Hartmann, E. Jansen, B. Kirtman, R. Knutti, K. Krishna Kumar, P. Lemke, J. Marotzke, V. Masson-Delmotte, G.A. Meehl, I.I. Mokhov, S. Piao, V. Ramaswamy, D. Randall, M. Rhein, M. Rojas, C. Sabine, D. Shindell, L.D. Talley, D.G. Vaughan and S.-P. Xie, 2013. Technical Summary. In: Climate Change 2013: The Physical Science Basis. Contribution of Working Group I to the Fifth Assessment Report of the Intergovernmental Panel on Climate Change [Stocker, T.F., D. Qin, G.-K. Plattner, M. Tignor, S.K. Allen, J. Boschung, A. Nauels, Y. Xia, V. Bex and P.M. Midgley (eds.)]. Cambridge University Press, Cambridge, United Kingdom and New York, NY, USA.

Suzuki, K., J.-C. Golaz and G.L. Stephens, 2013. Evaluating cloud tuning in a climate model with satellite observations. Geophys. Res. Lett., 40(16), doi: 10.1002/grl.50874

Tapley, B.D., S. Bettadpur, J.C. Ries, P.F. Thompson and M.M. Watkins, 2004. GRACE measurements of mass variability in the Earth system. Science, 305(5683), 503-505, doi: 10.1126/science.1099192

Tawfik, A.B., R. Stöckli, A. Goldstein, S. Pressley and A.L. Steiner, 2012. Quantifying the contribution of environmental factors to isoprene flux interannual variability. Atmospheric Environment, 54, 216224, doi: 10.1016/j.atmosenv.2012.02.018

Taylor, K.E., R.J. Stouffer and G.A. Meehl, 2012. An overview of CMIP5 and the experiment design. Bull. Amer. Meteor. Soc., 93, 485-498, doi: 10.1175/BAMS-D-11-00094.1

Tietsche, S., M.A. Balmaseda, H. Zuo and K. Mogensen, 2015. Arctic sea ice in the global eddypermitting ocean reanalysis ORAP5. Clim. Dyn., in press, doi: 10.1007/s00382-015-2673-3

Thompson, P.D., 1957. Uncertainty of initial state as a factor in the predictability of large scale atmospheric flow patterns. Tellus, 9, 275-295, doi: 10.1111/j.2153-3490.1957.tb01885.x

Tokarczyk, P., J.P. Leitao, J. Rieckermann, K. Schindler and F. Blumensaat, 2015. High-quality observation of surface imperviousness for urban runoff modelling using UAV imagery. Hydrol. Earth Syst. Sci. Discuss., 12, 1205-1245, doi: 10.5194/hessd-12-1205-2015

Trenberth, K.E., 2011. Changes in precipitation with climate change. Climate Research, 47, 123-138, doi: $10.3354 /$ cr00953

Trenberth, K.E., J.T. Houghton, and L.G. Meira Filho, 1996. The climate system: An overview. Chapter 1 of Climate Change 1995. The science of Climate Change. Contribution of WG 1 to the Second Assessment Report of the Intergovernmental Panel on Climate Change. J.T. Houghton, L.G. Meira Filho, B. Callander, N. Harris, A. Kattenberg, and K. Maskell (eds). Cambridge University Press. 51-64, ISBN 0521564360

Trenberth, K.E., and J.M. Caron, 2001. Estimates of meridional atmosphere and ocean heat transports. J. Clim., 14, 3433-3443, doi: 10.1175/1520-0442(2001)014<3433:EOMAAO>2.0.CO;2 
Trenberth, K. E., A. Dai, R. M. Rasmussen and D. B. Parsons, 2003. The changing character of precipitation. Bull. Amer. Meteor. Soc., 84, 1205-1217, doi: 10.1175/BAMS-84-9-1205

Trenberth, K.E., J.T. Fasullo and J. Kiehl, 2009. Earth's global energy budget. Bull. Amer. Meteor. Soc., 92, 311-323, doi: 10.1175/2008BAMS2634.1

Trenberth, K.E., J.T. Fasullo and J. Mackaro, 2011. Atmospheric moisture transports from ocean to land and global energy flows in reanalyses. J. Clim., 24, 4907-4924, doi: 10.1175/2011JCLI4171.1

Trenberth, K.E., and J.T. Fasullo, 2013. Regional energy and water cycles: Transports from ocean to land. J. Clim., 26, 7837-7851, doi: 10.1175/JCLI-D-00008.1

Uppala, S.M., P.W. Kållberg, A.J. Simmons, U. Andrae, V. da Costa Bechtold, M. Fiorino, J.K Gibson, J. Haseler, A. Hernandez, G.A. Kelly, X. Li, K. Onogi, S. Saarinen, N. Sokka, R.P. Allan, E. Andersson, K. Arpe, M.A. Balmaseda, A.C.M. Beljaars, L. van de Berg, J. Bidlot, N. Bormann, S. Caires, F. Chevallier, A. Dethof, M. Dragosavac, M. Fisher, M. Fuentes, S. Hagemann, E. Hólm, B.J. Hoskins, L. Isaksen, P.A.E.M. Janssen, R. Jenne, A.P. McNally, J.-F. Mahfouf, J.- J. Morcrette, N.A Rayner, R.W. Saunders, P. Simon, A. Sterl, K.E. Trenberth, A. Untch, D. Vasiljevic, P. Viterbo and J. Woollen 2005. The ERA-40 Reanalysis. Quart. J. Roy. Meteorol. Soc., 131, 2961-301, doi: 10.1256/qj.04.176

USDA-NRCS, National Engineering Handbook. Section 4 - Hydrology. Available from www.nrcs.usda.gov/wps/portal/nrcs/detailfull/national/water/?cid=stelprdb1043063

Vannière B., E. Guilyardi, G. Madec, F.J. Doblas-Reyes and S. Woolnough, 2013. Using seasonal hindcasts to understand the origin of the equatorial cold tongue bias in CGCMs and its impact on ENSO. Clim. Dyn., 40, 963-981, doi: 10.1007/s00382-012-1429-6

Vecchi, G.A., T. Delworth, R. Gudgel, S. Kapnick, A. Rosati, A.T. Wittenberg, F. Zeng, W. Anderson, V. Balaji, K. Dixon, L. Jia, H.-S. Kim, L. Krishnamurthy, R. Msadek, W.F. Stern, S.D. Underwood, G. Villarini, X. Yang and S. Zhang, 2014. On the Seasonal Forecasting of Regional Tropical Cyclone Activity. J. Climate, 27, 7994-8016, doi: 10.1175/JCLI-D-14-00158.1

Vernier, J.-P., L.W. Thomason, J.-P. Pommereau, A. Bourassa, J. Pelon, A. Garnier, A. Hauchecorne, L. Blanot, C. Trepte, Doug Degenstein and F. Vargas, 2011. Major influence of tropical volcanic eruptions on the stratospheric aerosol layer during the last decade. Geophys. Res. Lett., 38, L12807, doi: 10.1029/2011GL047563.

Wanninkhof, R., W.E. Asher, D.T. Ho, C. Sweeney and W.R. McGillis, 2009. Advances in quantifying air-sea gas exchange and environmental forcing. Annu. Rev. Mar. Sci., 1, 213-244, doi:

10.1146/annurev.marine.010908.163742

Wanninkhof, R., G.H. Park, T. Takahashi, R.A. Feely, J.L. Bullister and S.C. Doney, 2013. Changes in deep-water $\mathrm{CO}_{2}$ concentrations over the last several decades determined from discrete $\mathrm{pCO}_{2}$ measurements. Deep-Sea Res. Pt. I, 74, 48-63, doi:10.1016/j.dsr.2012.12.005

Wickert, J., O.B. Andersen, G. Beyerle, E. Cardellach, B. Chapron, C. Gommenginger, J. Hatton, P. Hoeg, A. Jäggi, N. Jakowski, M. Kern, T. Lee, M. Martín-Neira, O. Montenbruck, N. Pierdicca, A. Rius, C.K. Shum and C. Zuffada, 2014. Innovative Remote Sensing using the International Space Station: 
GNSS Reflectometry with GEROS. International Geoscience and Remote Sensing Symposium (IGARSS 2014) / 35th Canadian Symposium on Remote Sensing (35th CSRS), Quebec, Canada, 13-18 July 2014

Widlowski J.-L., B. Pinty, M. Clerici, Y. Dai, M. De Kauwe, K. de Ridder, A. Kallel, H. Kobayashi, T. Lavergne, W. Ni-Meister, A. Olchev, T. Quaife, S. Wang, W. Yang, Y. Yang and H. Yuan, 2011. RAMI4PILPS: An intercomparison of formulations for the partitioning of solar radiation in land surface models. Journal of Geophysical Research, 116, G02019, 25, doi: 10.1029/2010JG001511

Widlowski J.-L., B. Pinty, M. Lopatka, C. Atzberger, D. Buzica, M. Chelle, M. Disney, J. P. Gastellu Etchegorry, M. Gerboles, N. Gobron, E. Grau, H. Huang, A. Kallel, H. Kobayashi, P. Lewis., W. Qin, M. Schlerf, J. Stuckens and D. Xie, 2013. The fourth Radiation Transfer Model Intercomparison (RAMIIV): Proficiency Testing of Canopy Reflectance Models with ISO-13528. Journal of Geophysical Research - Atmospheres, 118(13), 6869-6890, doi: 10.1002/jgrd.50497

Wielicki, B.A., D.F. Young, M.G. Mlynczak, K.J. Thome, S. Leroy, J. Corliss, J.G. Anderson, C.O. Ao, R. Bantges, F. Best, K. Bowman, H. Brindley, J.J. Butler, W. Collins, J.A. Dykema, D.R. Doelling, D.R. Feldman, N. Fox, X. Huang, R. Holz, Y. Huang, Z. Jin, D. Jennings, D.G. Johnson, K. Jucks, S. Kato, D.B. Kirk-Davidoff, R. Knuteson, G. Kopp, D.P. Kratz, X. Liu, C. Lukashin, A.J. Mannucci, N.

Phojanamongkolkij, P. Pilewskie, V. Ramaswamy, H. Revercomb, J. Rice, Y. Roberts, C.M. Roithmayr, F. Rose, S. Sandford, E.L. Shirley, W.L. Smith Sr., B. Soden, P.W. Speth, W. Sun, P.C. Taylor, D. Tobin and X. Xiong, 2013. Achieving Climate Change Absolute Accuracy in Orbit. Bull. Amer. Meteor. Soc., 94, 1519-1539, doi: 10.1175/BAMS-D-12-00149.1

Wijffels, S.E., J. Willis, C.M. Domingues, P. Barker, N.J. White, A. Gronell, K. Ridgway and J.A. Church, 2008. Changing expendable bathythermograph fall rates and their impact on estimates of thermosteric sea level rise. J. Climate, 21, 5657-5672, doi: 10.1175/2008JCLI2290.1

Wild, M., D. Folini, C. Schär, N. Loeb, E.G. Dutton and G. König-Langlo, 2013. The global energy balance from a surface perspective. Clim. Dyn., 40, 3107-3134, doi: 10.1007/s00382-012-1569-8

Willis, J.K., J.M. Lyman, G.C. Johnson and J. Gilson, 2007. Correction to "Recent cooling of the upper ocean". Geophys. Res. Lett., 34, L16601, doi: 10.1029/2007GL030323

Winiarek, V., M. Bocquet, N. Duhanyan, Y. Roustan, O. Saunier and A. Mathieu, 2014. Estimation of the caesium-137 source term from the Fukushima Daiichi nuclear power plant using a consistent joint assimilation of air concentration and deposition observations. Atmos. Env., 62, 268-279, doi: 10.1016/j.atmosenv.2013.10.017

World Economic Forum, 2011. Global Risks 2011. 6th ed. Available from www.weforum.org/reports/global-risks-report-2011

WMO, 2012. Final Report of the Fifth WMO Workshop on the Impact of Various Observing Systems on Numerical Weather Prediction. World Meteorological Organization Technical Report no. 2012-1, $25 \mathrm{pp}$. Available together with presentations from www.wmo.int/pages/prog/www/OSY/Reports/NWP-5_Sedona2012.html

WMO, 2013. Sub-seasonal to Seasonal Prediction - Research Implementation Plan. 63pp. Available from www.wmo.int/pages/prog/arep/wwrp/new/S2S_project_main_page.html 
WMO, 2014. Report of GRUAN-GSICS-GNSSRO WIGOS Workshop on Upper-Air Observing System Integration and Application. Geneva, 6-8 May 2014, 59pp. Available from www.wmo.int/pages/prog/www/WIGOS-WIS/reports.html

Xue, Y., M.A. Balmaseda, T. Boyer, N. Ferry, S. Good, I. Ishikawa, A. Kumar, M. Rienecker, A.J. Rosati and Y. Yin, 2012. A Comparative Analysis of Upper-Ocean Heat Content Variability from an Ensemble of Operational Ocean Reanalyses. J. Climate, 25, 6905-6929, doi: 10.1175/JCLI-D-11-00542.1

Yang, D., Y. Zhou and Y. Wang, 2009. Remote Sensing with Reflected Signals. InsideGNSS, Sep/Oct 2009, pg. 40-45. Available from www.insidegnss.com

Yin, C., E. Lopez-Baeza, M. Martin-Neira, R. Fernández Morán, N. Peinado-Galán, E. Navarro, A. Egido, A. Mollfulleda, W. Li, Y. Cao, B. Zhu and B.D. Yang, 2015. Soil Moisture Remote Sensing with GNSS-R at the Valencia Anchor Station. 2nd SMOS Science Conference, ESA-ESAC, Villafranca, Madrid, Spain, 25-29 May 2015. Available from www.smos2015.info

Yu, F., 2010. Ion-mediated nucleation in the atmosphere: Key controlling parameters, implications, and look-up table. J. Geophys. Res., 115, D03206, doi: 10.1029/2009JD012630

Zaehle, S., and D. Dalmonech, 2011. Carbon-nitrogen interactions on land at global scales: Current understanding in modelling climate biosphere feedbacks. Curr. Opin. Environ. Sustain., 3, 311-320, doi: $10.1016 / \mathrm{j}$. cosust.2011.08.008

Zaehle, S. P. Ciais, A.D. Friend and V. Prieur, 2011. Carbon benefits of anthropogenic reactive nitrogen offset by nitrous oxide emissions. Nature Geosci., 4, 601-605, doi: 10.1038/ngeo1207

Zhang, S., M.J. Harrison, A.J. Rosati and A.T. Wittenberg, 2007. System Design and Evaluation of Coupled Ensemble Data Assimilation for Global Oceanic Climate Studies. Monthly Weather Review, 135, 3541-3564, doi: 10.1175/MWR3466.1

Zhou, C., and J.E. Penner, 2014. Aircraft soot indirect effect on large-scale cirrus clouds: Is the indirect forcing by aircraft soot positive or negative? J. Geophys. Res. Atmos., 119, 11,303-11,320, doi: 10.1002/2014JD021914

Zhu, J., B. Huang, R.-H. Zhang, Z.-Z. Hu, A. Kumar, M.A. Balmaseda, L. Marx and J.L. Kinter III, 2014. Salinity anomaly as a trigger for ENSO events. Scientific Reports, 4, 6821, doi: 10.1038/srep06821 


\section{Appendix: List of names and acronyms}

AATSR

ADM-Aeolus

AERONET

AIRS

Altika

AMIP

AMSR

AMSU-A

AR5

Argo

ARM

Aqua

Aquarius

ASCAT

ASTER

Aura

AVHRR

BUV

CALIPSO

CCDAS

$\mathrm{CCl}$

$\mathrm{CCN}$

CEOS

CERES

CFMIP

CGMS

CLARREO

Cloudsat

CMC

CMIP

CONTRAIL

Copernicus

cosMIC

COSPAR

CrIS

Cryosat

CTD

DEM

DORIS

DMSP

EarthCARE

ECMWF

ECV
Advanced Along-Track Scanning Radiometer on Envisat

Atmospheric Dynamics Mission for wind measurement from space

AErosol RObotic NETwork for ground-based remote sensing of aerosol

Atmospheric Infrared Sounder on Aqua satellite

Altimeter for sea-level measurement

Atmospheric Model Intercomparison Project

Advanced Microwave Scanning Radiometer flown on several satellites

Advanced Microwave Sounding Unit A, flown on several satellites

Fifth Assessment Report of the IPCC

Global array of free-drifting ocean profiling floats

Atmospheric Radiation Measurement programme

Satellite of the EOS programme

Ocean-salinity and soil-moisture measuring satellite on SAC-D satellite

Advanced Scatterometer on Metop satellites

Advanced Spaceborne Thermal Emission and Reflection Radiometer on Terra

Satellite of the EOS programme

Advanced Very High Resolution Radiometer on NOAA-n and Metop satellites

Backscatter Ultraviolet spectrometer on early satellites

Cloud-Aerosol Lidar and Infrared Pathfinder Satellite Observations

Carbon Cycle Data Assimilation System

Climate Change Initiative of ESA

Cloud Condensation Nucleus

Committee on Earth Observation Satellites

Clouds and the Earth's Radiant Energy System satellite-borne instrument

Cloud Feedback Model Intercomparison Project

Coordination Group for Meteorological Satellites

Climate Absolute Radiance and Refractivity Observatory

Satellite making advanced cloud observations using radar

Canadian Meteorological Centre

Coupled Model Intercomparison Project

Comprehensive Observation Network for Trace gases by Airliner

European Earth observation programme

Constellation Observing System for Meteorology, Ionosphere, and Climate, a mission comprising a set of GPS-receiver satellites

Committee on Space Research

Cross-track Infrared Sounder on JPSS satellites

Radar imaging satellite focussed on ice measurement

Conductivity, Temperature and Depth

Digital Elevation Model

Doppler Orbitography and Radio-positioning Integrated by Satellite

Defense Meteorological Satellite Program of the USA

Joint European/Japanese satellite for observing clouds, aerosols and radiation European Centre for Medium-Range Weather Forecasts

Essential Climate Variable 


\begin{tabular}{|c|c|}
\hline ENSO & El Niño Southern Oscillation \\
\hline Envisat & Environmental satellite of ESA \\
\hline EOS & Earth Observing System programme of NASA \\
\hline ERA & European (or ECMWF) Reanalysis \\
\hline ERS & European Remote Sensing satellites of ESA \\
\hline ESA & European Space Agency \\
\hline ET & Evapotranspiration \\
\hline EUMETSAT & European Organization for the Exploitation of Meteorological satellites \\
\hline FAPAR & Fraction of Absorbed Photosynthetically Active Radiation \\
\hline FLUXNET & Network of micrometeorological tower measurement sites \\
\hline Future Earth & Multi-sponsored research platform supporting transformation sustainability \\
\hline FY-3 & Fengyun-3, a series of Chinese polar-orbiting meteorological satellites \\
\hline GARP & Global Atmospheric Research Programme \\
\hline GAW & Global Atmosphere Watch programme of WMO \\
\hline GCOM-C, W & JAXA's Global Change Observation Missions for Carbon and Water \\
\hline GCOS & Global Climate Observing System \\
\hline GEO & Group on Earth Observations \\
\hline \multirow[t]{2}{*}{ GEMS } & Geostationary Environment Monitoring Spectrometer mission of the Korea \\
\hline & Aerospace Research Institute \\
\hline GEO-CAPE & GEOstationary Coastal and Air Pollution Events mission under development \\
\hline GEOS & NASA Goddard Earth Observing System model \\
\hline GEOSS & Global Earth Observation System of Systems \\
\hline GEROS-ISS & $\begin{array}{l}\text { GNSS Reflectometry, Radio Occultation and Scatterometry experiment, on the } \\
\text { International Space Station }\end{array}$ \\
\hline GFDL & Geophysical Fluid Dynamics Laboratory of NOAA \\
\hline GGOS & Global Geodetic Observing System \\
\hline GHRSST & Group for High-Resolution Sea Surface Temperature \\
\hline GLOSS & Global Sea-Level Observing System \\
\hline GNSS & Global Navigation Satellite System \\
\hline GOCE & ESA's Gravity field and steady-state Ocean Circulation Explorer \\
\hline $\mathrm{GOCl}$ & Korea's Geostationary Ocean Colour Imager \\
\hline GOME & Global Ozone Monitoring Experiment instruments on ERS-2 and Metop \\
\hline GOOS & Global Ocean Observing System \\
\hline GOSAT & Greenhouse gases Observing SATellite of JAXA \\
\hline GO-SHIP & Global Ocean Ship-based Hydrographic Investigations Program \\
\hline GPCC & Global precipitation Climatology Centre \\
\hline GPCP & Global Precipitation Climatology Project \\
\hline GPM & Global Precipitation Measurement mission \\
\hline GRACE & Gravity Recovery and Climate Experiment mission of NASA \\
\hline GRIFEX & $\begin{array}{l}\text { GEO-CAPE Read-Out Integrated Circuit In-Flight Performance Experiment } \\
\text { cubesat mission }\end{array}$ \\
\hline GRUAN & GCOS Reference Upper-Air Network \\
\hline GSICS & Global Space-based Inter-Calibration System \\
\hline IAGOS & In-Service Aircraft for a Global Observing System \\
\hline IASI & Infrared Atmospheric Sounding Interferometer on Metop \\
\hline
\end{tabular}




\begin{tabular}{|c|c|}
\hline ICESat & Ice, Cloud and land Elevation Satellite of NASA \\
\hline ICSU & International Council for Science \\
\hline IGBP & International Geosphere-Biosphere Programme \\
\hline IMPROVE & Interagency Monitoring of Protected Visual Environments project \\
\hline $10 C$ & Intergovernmental Oceanographic Commission of UNESCO \\
\hline IPCC & Intergovernmental Panel on Climate Change \\
\hline ISCCP & International Satellite Cloud Climatology Project \\
\hline ISMN & International Soil Moisture Network \\
\hline Jason-n & Satellite series measuring ocean surface topography \\
\hline JAXA & Japan Aerospace Exploration Agency \\
\hline JMA & Japan Meteorological Agency \\
\hline JPS & Joint Polar System (of meteorological satellites) \\
\hline JRA & Japanese Reanalysis \\
\hline JPSS & US Joint Polar Satellite System for operational environmental observation \\
\hline LAI & Leaf Area Index \\
\hline Landsat & Series of US land-imaging satellites \\
\hline LULCC & Land Use and Land Cover Change \\
\hline MeASURES & $\begin{array}{l}\text { Making Earth System Data Records for Use in Research Environments } \\
\text { programme of NASA }\end{array}$ \\
\hline Megha-Tropiques & French/Indian satellite to study the water cycle in the tropical atmosphere \\
\hline MERIS & MEdium Resolution Imaging Spectrometer on Envisat \\
\hline MERRA & Modern-Era Retrospective Analysis for Research and Applications \\
\hline Meteosat & Series of European geostationary satellites \\
\hline Metop & Meteorological operational polar-orbiting satellite series of EUMETSAT \\
\hline Metop-SG & Second-generation Metop series \\
\hline MLS & Microwave Limb Sounder on Aura \\
\hline MISR & Multi-angle Imaging SpectroRadiometer on Terra satellite \\
\hline MODIS & Moderate Resolution Imaging Spectroradiometer on Aqua and Terra satellites \\
\hline MOPITT & Measurement of Pollution in the Troposphere instrument on Terra \\
\hline NASA & US National Aeronautics and Space Administration \\
\hline NCAR & US National Center for Atmospheric Research \\
\hline NCEP & National Centers for Environmental Prediction of NOAA \\
\hline NMME & North American Multi-Model Ensemble \\
\hline NOAA & US National Oceanic and Atmospheric Administration \\
\hline NOAA-n & Polar-orbiting satellite in the operational meteorological programme of NOAA \\
\hline NRC & US National Research Council \\
\hline NWP & Numerical Weather Prediction \\
\hline Oceansat & Indian satellite providing ocean colour and scatterometer measurements \\
\hline OCM & Ocean Colour Monitor instrument on Oceansat \\
\hline $\mathrm{OCO}$ & Orbiting Carbon Observatory of NASA \\
\hline Odin & Satellite of Swedish-led international mission \\
\hline $\mathrm{OLCl}$ & Ocean and Land Colour Instrument on Sentinel-3 \\
\hline OMI & Ozone Monitoring Instrument on the Aura satellite \\
\hline OMPS & Ozone Mapping and Profiler Suite instrument on Suomi NPP and JPSS satellites \\
\hline ORAS4 & Ocean Reanalysis using ECMWF's coupled ocean-atmosphere System 4 \\
\hline
\end{tabular}


OSCAR

PARASOL

PICASSO

POLDER

PROBA

QuikSCAT

RACE

RAPID

RAVAN

RCP

SAC-D

SAR

SARAL

SBA

SBUV

SCIAMACHY

SCOPE-CM

SeaWIFS

Sentinel-n

SEVIRI

SGLI

SIMBA

SLA

SLSTR

SMAP

SMMR

SMOS

SORCE

SRTM

SSM/I

SSS

SST

Suomi NPP

SWOT

TanDEM-X

TAO/TRITON

TCCON

TCRE
Observing Systems Capability Analysis and Review tool of WMO

Polarization and Anisotropy of Reflectances for Atmospheric Sciences coupled with Observations from a Lidar, a French mission observing clouds and aerosols PICosatellite for Atmospheric and Space Science Observations POLarization and Directionality of the Earth's Reflectances instrument on PARASOL

Project for On-Board Autonomy series of microsatellites operated by ESA NASA scatterometer mission

Radiometer Atmospheric CubeSat Experiment

Observing array for the Atlantic meridional overturning circulation

Radiometer Assessment using Vertically Aligned Nanotubes cubesat mission Representative Concentration Pathway

Satelite de Aplicaciones Cientificas-D, an Argentinian satellite flying the NASA instrument Aquarius

Synthetic Aperture Radar

Satellite with ARGOS data collection system and Altika

Societal Benefit Area (of the GEOSS)

Solar Backscatter Ultraviolet instrument on NOAA-n satellites

SCanning Imaging Absorption spectroMeter for Atmospheric CHartographY on Envisat

Sustained and Coordinated Processing of Environmental Satellite data for Climate Monitoring

Sea-Viewing Wide Field-of-View satellite-borne Sensor

Series of Earth-observation satellites of the Copernicus programme

Spinning Enhanced Visible and InfraRed Imager on EUMETSAT geostationary satellites

Second Generation Global Imager on GCOM-C satellites

Sun-earth IMBAlance radiometer cubesat mission

Sea-level anomaly

Sea and Land Surface Temperature Radiometer on Sentinel-3

Soil Moisture Active Passive NASA satellite

Scanning Multichannel Microwave Radiometer on NASA's Nimbus-7 satellite

Soil Moisture and Ocean Salinity mission of ESA

Solar Radiation and Climate Experiment mission of NASA

Shuttle Radar Topography Mission

Special Sensor Microwave Imager instrument on DMSP satellites

Sea-surface salinity

Sea-surface temperature

Suomi National Polar-orbiting Partnership, first satellite in the JPSS series

Surface Water Ocean Topography mission

Synthetic aperture radar satellite twinned with TerraSAR-X for digital elevation modelling

Array of moored buoys in the tropical Pacific Ocean

Total Carbon Column Observing Network

Transient Climate Response to cumulative $\mathrm{CO}_{2}$ emissions 


$\begin{array}{ll}\text { TEMPEST-D } & \text { Temporal Experiment for Storms and Tropical Systems - Demonstrator cubesat } \\ \text { TEMPO } & \text { mission } \\ \text { TES } & \text { Tropospheric Emissions: Monitoring of Pollution mission of NASA } \\ \text { Terra } & \text { Tropospheric Emission Spectrometer on Aura } \\ \text { TerraSAR-X } & \text { Satellite of the EOS programme } \\ \text { TOMS } & \text { Synthetic Aperture radar satellite } \\ \text { Topex/Poseidon } & \text { Total Ozone Mapping Spectrometer on multiple satellites } \\ \text { TPOS } & \text { Satellite mission to map ocean surface topography } \\ \text { TRMM } & \text { Tropical Pacific Observing System } \\ \text { TROPOMI } & \text { Tropical Rainfall Measuring Mission } \\ \text { 3MI } & \text { Tropospheric Monitoring Instrument, extending the capabilities of OMI } \\ & \text { Multi-viewing, Multi-channel, Multi-polarization Imaging instrument on Metop- } \\ \text { UNEP } & \text { SG } \\ \text { UNESCO } & \text { United Nations Environment Programme } \\ \text { UNFCCC } & \text { United Nations Educational, Scientific and Cultural Organization } \\ \text { VIIRS } & \text { United Nations Framework Convention on Climate Change } \\ \text { WCRP } & \text { Visible Infrared Imaging Radiometer Suite on NPP and JPSS satellites } \\ \text { WMO } & \text { World Climate Research Programme } \\ \text { WOCE } & \text { World Meteorological Organization } \\ \text { XBT } & \text { World Ocean Circulation Experiment } \\ & \text { Expendable bathythermograph }\end{array}$

